\title{
Supporting Self-Experimentation of Behavior Change Strategies
}

by

Jisoo Lee

\section{A Dissertation Presented in Partial Fulfillment of the Requirements for the Degree \\ Doctor of Philosophy}

Approved July 2016 by the Graduate Supervisory Committee:

Winslow Burleson, Chair

Eric B. Hekler

David Tinapple

Erin Walker

\section{ARIZONA STATE UNIVERSITY}

December 2016 


\begin{abstract}
Desirable outcomes such as health and wellbeing are tightly linked to people's behaviors, thus inspiring research on technologies that support productively changing those behaviors. Many behavior change technologies are designed by Human-Computer Interaction experts, but this approach makes it difficult to personalize support to each user's unique goals and needs. As an alternative to the provision of expert-developed pre-fabricated behavior change solutions, the present study aims to empower users' selfexperimentation for behavior change. To this end, two levels of supports were explored. First, the provision of interactive digital materials to support users' creation of behavioral plans was developed. In the initial step, a tutorial for self-experimentation for behavior change that was fully scripted with images in succession was created. The tutorial focuses on facilitating users' learning and applying behavior change techniques. Second, users were equipped with a tool to support their implementation of contextaware just-in-time interventions. This tool enables prototyping of sensor-based responsive systems for home environments, integrating simple sensors (two-state magnetic sensors, etc.) and media event components (wireless sound, etc.).
\end{abstract}

To evaluate the effectiveness of these two approaches, a between-subject trial comparing the approaches to a sleep education control was conducted with 27 participants over 7 weeks. Although results did not reveal significant difference in sleep quality improvement between the conditions, trends indicating greater effectiveness in the two treatment groups were observed. Analysis of the plans participants created and their revision performance also indicated that the two treatment groups developed more specific and personalized plans compared with the control group. 


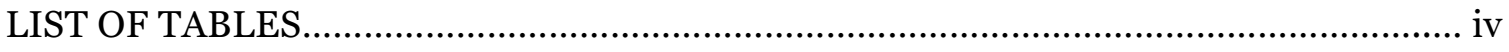

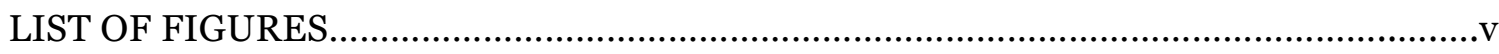

CHAPTER

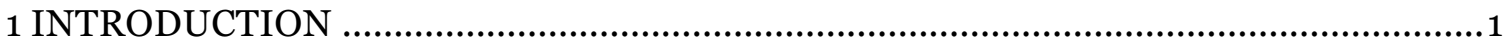

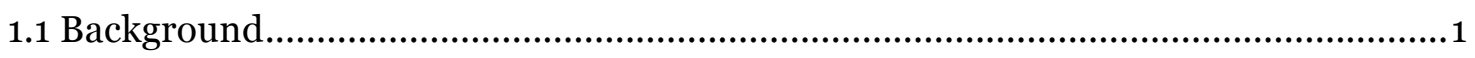

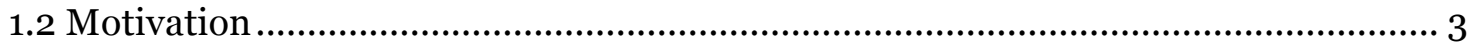

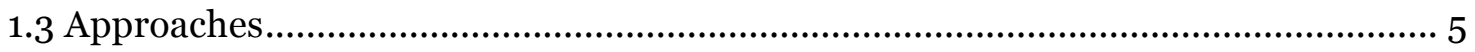

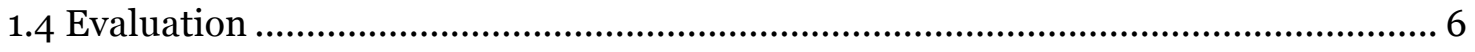

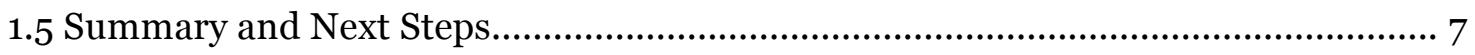

2 BACKGROUND AND RELATED WORK …........................................................ 9

2.1 Self-Regulated Behavior ........................................................................... 9

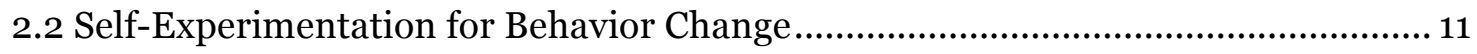

2.3 Support for Creation of Behavior Change Plans...............................................14

2.4 End-User Creation of Context-Aware Applications ............................................19

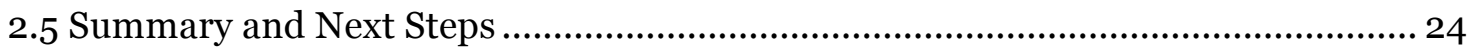

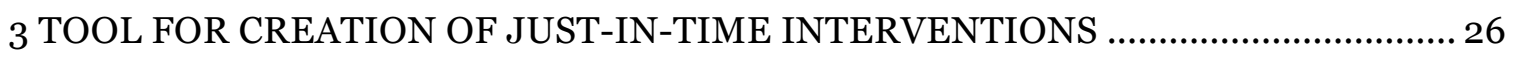

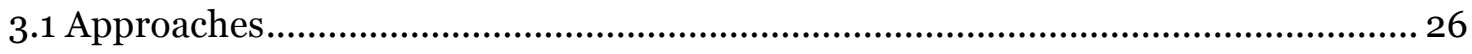

3.2 Architecture and Applications .................................................................. 29

3.4 User Study of the Programming Interface ....................................................... 42

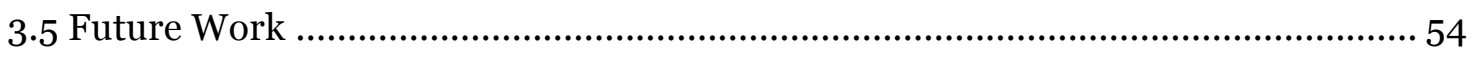

3.6 Summary and Next Steps ..........................................................................57 
4 SUPPORT FOR BEHAVIORAL PLAN CREATION ................................................. 59

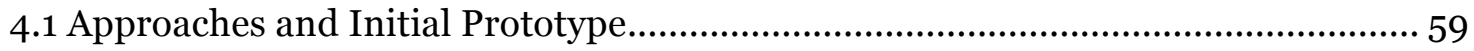

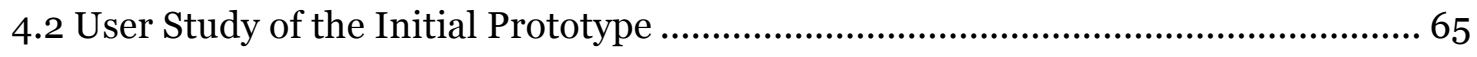

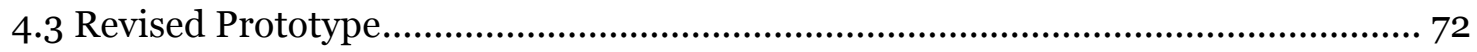

4.4 User Study of the Revised Prototype …......................................................... 78

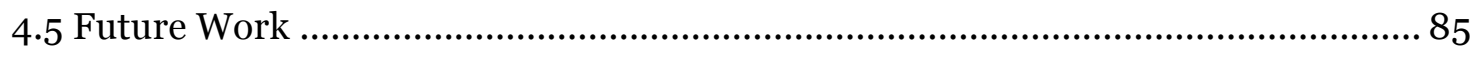

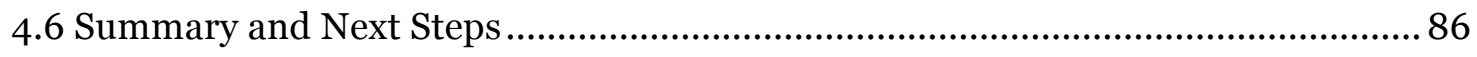

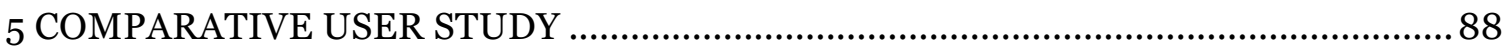

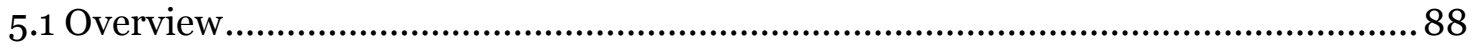

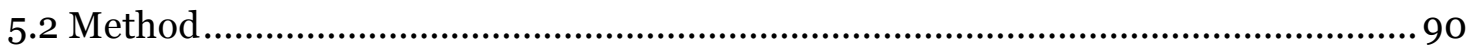

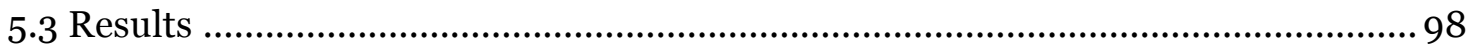

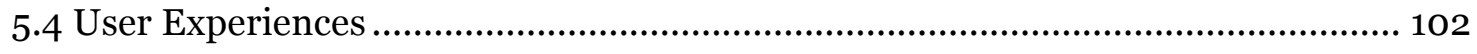

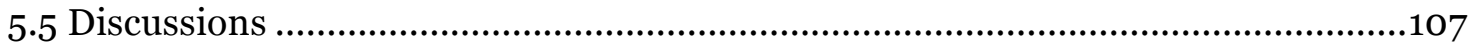

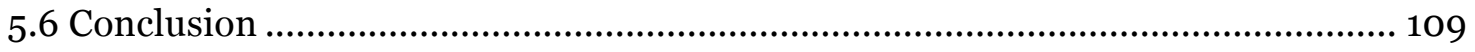

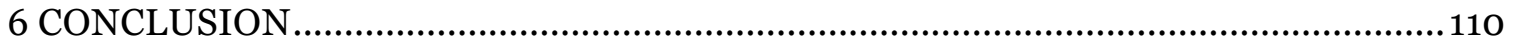

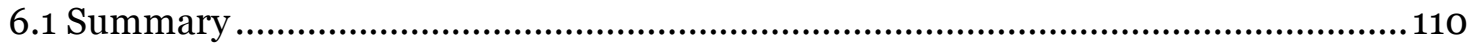

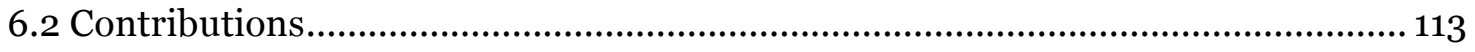

6.3 Limitations and Future Work ...................................................................... 115

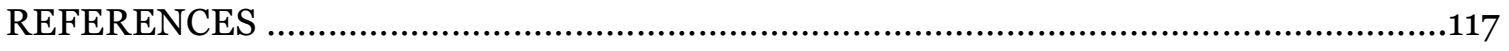




\section{LIST OF TABLES}

Table

Page

1. Descriptive Statistics for Ease of Use and Engagement ....................................... 48

2. User Study of the Initial Prototype of a Self-experimentation for Behavior Change

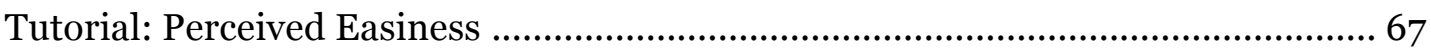

3. User Study of the Initial Prototype of a Self-experimentation for Behavior Change

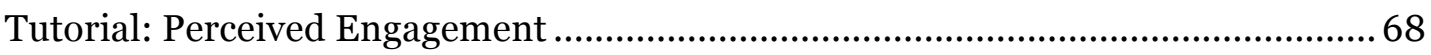

4. User Study of the Initial Prototype of a Self-experimentation for Behavior Change

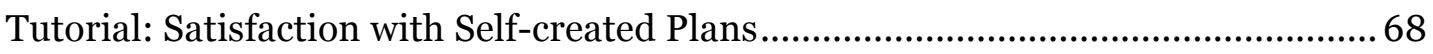

5. Exemplar Participants-generated Plans of the Initial Prototype User Study .............. 69

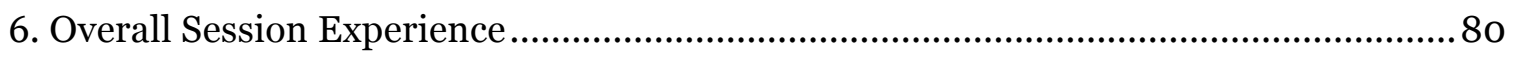

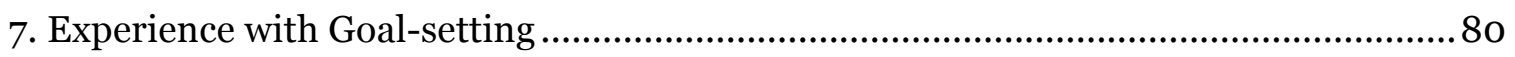

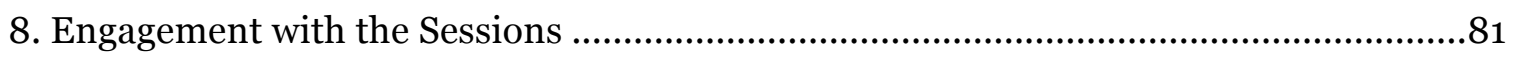

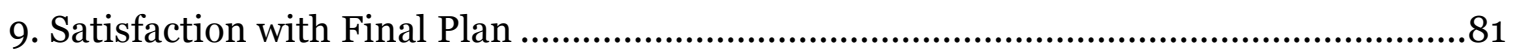

10. Follow-up of Screening Survey Respondents ..................................................... 95

11. Age and Gender Distribution of Participants ...................................................... 97

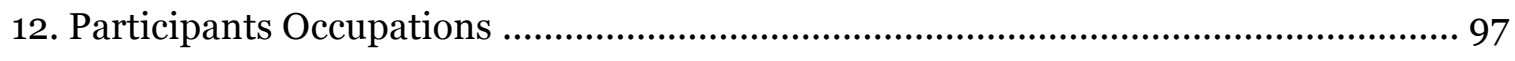

13. Sleep Issues and Numbers of Participants............................................................ 98

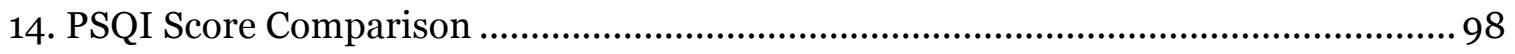

15. Daily Satisfaction Rating Change …................................................................. 101

16. Exemplar Behavioral Plans in Pursuing “Adjusting/Sticking to a Sleep Schedule” . 102 


\section{LIST OF FIGURES}

Figure $\quad$ Page

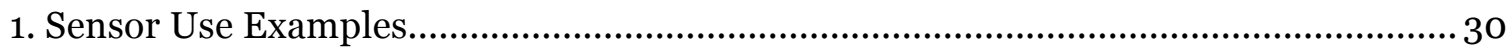

2. Example of X10 Door Sensor Transformation …................................................... 30

3. Example of X10 Motion Sensor Transformation....................................................... 30

4. GaLLaG Construction Tool Architecture ............................................................... 31

5. Main and Application List Screens of GaLLaG Strip............................................. 38

6. Adding an Action Frame in Recording Mode ....................................................... 39

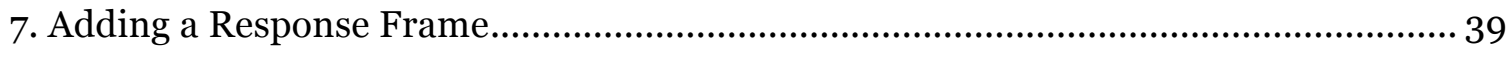

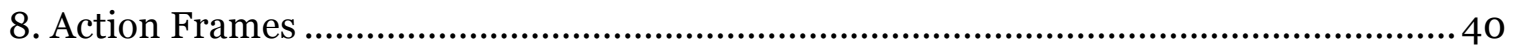

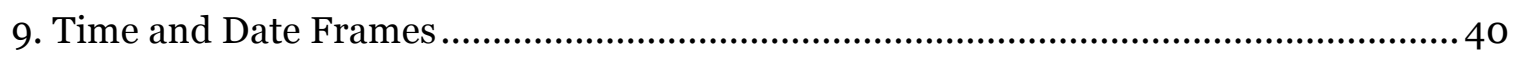

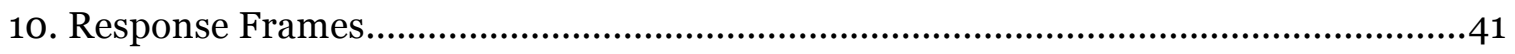

11. Action Frame after Being Customized .............................................................41

12. Living Room Setting Built for GaLLaG Strip User Study ....................................... 43

13. Manually Adding a Motion Detected Action........................................................... 43

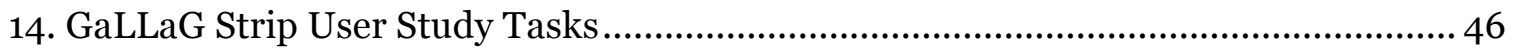

15. Initial Prototype of the Tutorial for Supporting Behavioral Plan Creation: Setting a

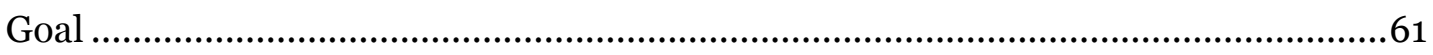

16. Initial Prototype of the Tutorial for Supporting Behavioral Plan Creation: Making

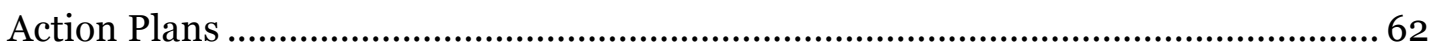

17. Initial Prototype of the Tutorial for Supporting Behavioral Plan Creation: Exemplar

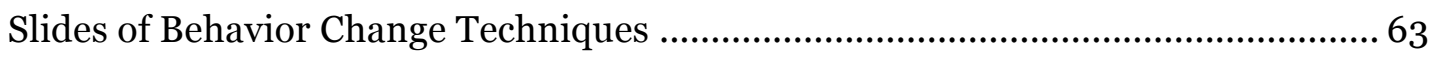

18. Initial Prototype of the Tutorial for Supporting Behavioral Plan Creation: Self-

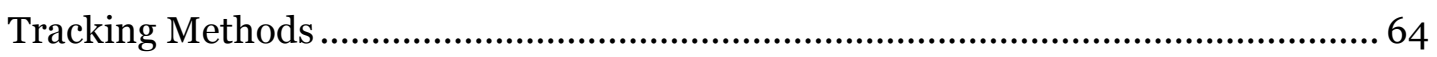


Figure

19. Frequency of Behavior Change Techniques Chosen .............................................. 70

20. Revised Prototype of the Behavioral Plan Creation Support: Setting Goals ...............75

21. Revised Slides of Behavior Change Techniques.........................................................77

22. Slide for Self-diagnosis with the Meta-model ..........................................................77

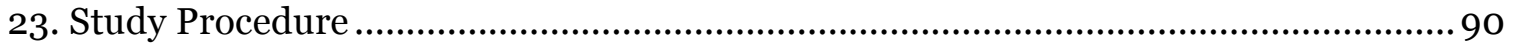

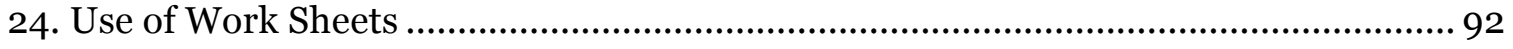

25. Example Ideation of GaLLaG Applications ........................................................... 92

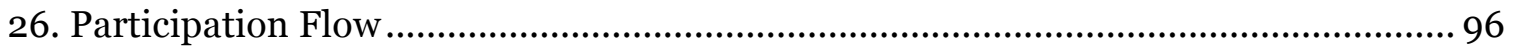

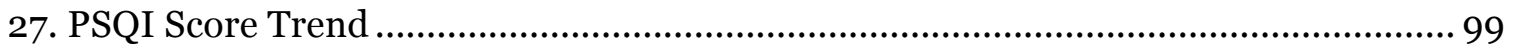

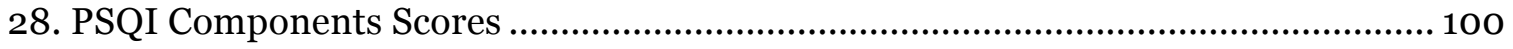

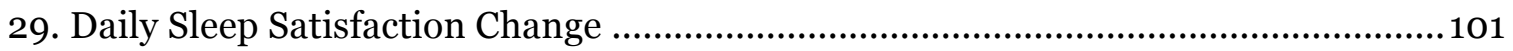




\section{INTRODUCTION}

\subsection{Background}

\section{Behavior Change for Life Satisfaction}

Extensive evidence demonstrates the importance of engagement in particular behaviors for better health, productivity, and wellbeing (de Ridder \& de Wit, 20o8; Nelson et al., 2007; Lindner, Menzies, Kelly, Taylor, \& Shearer, 2003). For example, daily tooth brushing is recommended for oral health (Attin \& Hornecker, 2005) and regular physical activity is acknowledged to reduce risk of cardiovascular disease, obesity, and colon cancer (Nelson et al., 2007). In addition, patients with type 2 diabetes should sustain self-care activities such as monitoring glucose level, taking medication, engaging in physical activity, implementing dietary changes, etc. (Funnell et al., 2008). Outside the health domain, several time management practices (e.g., making a to-do list, prioritizing tasks) are recommended to students for better academic achievement (Britton \& Glynn, 1989; Britton \& Tesser, 1991).

Recognition of the significant influence of daily activities has lead to comprehensive support to encourage initiation and maintenance of beneficial behaviors. For instance, informational resources and campaigns to promote weight loss are ubiquitous (e.g., 'Campaign to End Obesity'1). Likewise, many countries have government-led programs to promote smoking cessation (e.g., United States, 'Smokefree.gov'2). In addition, healthcare professionals deliver patient education, attempting to meet patients' variant

\footnotetext{
${ }^{1}$ http://www.obesitycampaign.org

${ }^{2}$ http://smokefree.gov/
} 
situations (different needs, attitudes, emotional responses, learning proficiency, etc.)(Lorig, 2001).

Despite such efforts, failure in sustaining desired behavior appears prevalent. For instance, Dunbar-Jacob and Mortimer-Stephens (2001) revealed that as many of as 60\% of persons with chronic disorders adhered poorly to medication regimens. According to the US census in 2010 (http://www.cdc.gov/nchs/), only $20.8 \%$ of adults (18 years of age and over) met the Physical Activity Guidelines for both aerobic physical and musclestrengthening activity recommended by the Office of Disease Prevention and Health Promotion (http://health.gov/paguidelines/).

\section{Behavior Change Technologies}

Driven by the importance of behavior change and the struggle to achieve it, the field of HCI (Human-Computer Interaction) has been increasingly exploring the use of computing technology to support behavior change (Fogg, 2002; Hekler, Klasnja, Froehlich, \& Buman, 2013). Particularly, increased availability of advanced ubiquitous and context-aware computing (Lyytinen \& Yoo, 2002) prompted attention to the issue. Exploring a wide range of daily life issues ranging from physical and emotional health (Maitland \& Chalmers, 2010; Nawyn, Intille, \& Larson, 2006) to energy conservation (Bang, Gustafsson, \& Katzeff, 2007; Bonanni, Arroyo, C. Lee, \& Selker, 2005), a variety of approaches has been proposed, for instance, persistent and objective monitoring using wearable sensor devices (Klasnja, Consolvo, McDonald, Landay, \& Pratt, 2009), support of self-discovery with information management systems (Kobsa, Chen, \& Wang, 2012), facilitation of social support with online social network sites (Newman, Lauterbach, Munson, Resnick, \& Morris, 2011), use of ambient displays for persistent and unobtrusive feedback (Jafarinaimi, Forlizzi, Hurst, \& Zimmerman, 2005), location- 
based smart phone gaming to promote outdoor activities (Spiesberger et al., 2015), and coaching services using affective or relational agents (Schulman \& Bickmore, 2009).

\subsection{Motivation}

Although substantial attention has been given to people's behavior change in the HCI community, most approaches were provisions of pre-fabricated solutions designed and implemented by experts, which may be problematic considering plausible difference between individuals' needs (Hekler et al., 2016b). For instance, Räisänen, Oinaskukkonen, \& Pahnila (2008) investigated opportune moments to display warning pictures about the dangers of smoking to people, and found that the optimal timing varied between individuals. King et al. (2013) developed three smartphone applications focused on improving mid-life and older adult's physical activity. The applications included a more game-like application focused on increasing positive emotions for activity, a socially oriented app focused on increasing awareness of the activity of others, and a rationally driven app focused on helping individuals set goals and track progress. Results indicated success at increasing physical activity with each application, but also found preferences among the users that shifted over time. For example, many individuals requested a "mix-and-match" approach at different times. While these prespecified tools were useful, formative interviews reinforced the need for strategies to facilitate personalization over time.

An alternative and complementary approach to enable more personalized and precise behavior change could be to help individuals create their own behavior change plans. This self-creation approach is supported by the Quantified Self (QS) movement (http://www.quantifiedself.com) where individuals work to understand themselves better through self-tracking and other self-study methods including ones that they create 
themselves (Li, Dey, \& Forlizzi, 2011; Choe et al., 2014). For instance, with intention to improve his irritable bowel syndrome symptoms, a person logs their eating, stress level, and symptoms three times a day in a self-created spreadsheet and explores how these factors might be related to one another in their situation. Choe and her colleagues (Choe, N. Lee, B. Lee, Pratt, \& Kientz, 2014) found that people who have adopted QS (QSers) often described their process of seeking answers as self-experimentation, and aim to discover meaningful self-knowledge that matters to them. With acknowledgement of genetic and environmental differences and the significance of personalized approaches to their issues (Williams, Willard, \& Snyderman, 2003), such practice undertaken by individuals appears valuable as it may produce rich personal knowledge critical in designing inventions to better treat issues. Karkar et al. (2015) proposed a framework to promote QSers' more systematic test and acquisition of more robust conclutions. Inspired by QSers' self-motivated efforts for self-improvement (Choe et al., 2014) and the value of personal and precise knowledge in obtaining effective interventions of issues, I envisioned that self-experimentation could be also possible and beneficial in people's acquisition of behavior-change plans.

The existing self-experimentation of QSers strengthens their behavior change because it allows them to discover behaviors critical to their diverse personal issues (e.g., avoiding spicy foods as it seems to cause my irritable bowel syndrome), which may differ between individuals. However, the need to extend the concept of self-experimentation to a systematic study to obtain behavior change plans, which differ from person to person, is acknowledged in the present research. After a person knows what they "should" do, a separate process is necessary to study how to change and maintain the targeted behavior over time. The former is termed self-experimentation for self-discovery, and the latter, 
self-experimentation for behavior change. Extensive work highlights a gap between what individuals intend to do compared to what they actually do (Sheeran, 2002), which confirms the need for self-experimentation for behavior change. The present research aims to empower users as they pursue the resolution to implement personally salient home-based behavior (i.e., sitting and watching TV, snacking, or flossing), with tools that help them to invent and test behavior change plans. Users can obtain plans optimized to their uniquely personal needs through such a journey, which may result in improvement in their issues of interest. In addition, the value of users' self-experimentation for behavior change is explored, and support to make such effort more fruitful is pursued.

\subsection{Approaches}

In pursuing supports for users' creation and test of behavior change plans, two levels of supports were developed. First, the provision of interactive digital materials that support users' creation of behavioral plan were devised with a particular focus on the benefit of users actively applying behavior change techniques, which are "observable, replicable, and irreducible component[s] of a [behavioral] intervention designed to alter or regulate behavior; that is, a technique is proposed to be an 'active ingredient' (e.g., feedback, selfmonitoring, and reinforcement)(Michie et al, 2013). It was considered that users might produce plans potentially effective in facilitating fulfillment of goals, by incorporating existing behavior change techniques validated with evidence-based research. Through iterative design processes, a self-experimentation for behavior change tutorial administered by a research assistant was developed. The tutorial could eventually be used to design interactive digital materials allowing users' sole work.

Second, users were equipped with a toolkit that enables implementation of contextaware just-in-time (JIT) interventions (Nahum-Shani, Hekler, \& Spruijt-Metz, 2015; 
Nawyn et al., 2006). The basic principle of JIT interventions is to provide triggers to engage in a desired behavior during states when a person has both the opportunity to engage in the behavior and the receptivity to interact with the system (Nahum-Shani et al., 2015). With the advancement of computing technologies such as networking, sensing, mobile devices, and information appliances, HCI researchers and designers have proposed JIT interventions that can deliver more adaptive content at more opportune moments (Nawyn et al., 2006; Arroyo, Bonanni, \& Selker, 2005). However, the users' own creation approach is considered logical because JIT strategies are often highly context-sensitive and idiosyncratic (Hekler et al., 2016b). For example, if a person is trying to improve their diet, a JIT intervention requires insights on when, where, with whom, and in what state (e.g., stress-eating) a person may be in when they eat too much to prompt during the JIT state. From this information, it can be determined when a prompt would be helpful for reducing over-eating. A construction tool that allows users to build sensor-based feedback systems at their home for JIT interventions was created by adopting an existing platform, GaLLaG (Game as Life, Life as Game), developed by the research group Motivational Environments (Burleson, Ruffenach, Jensen, Bandaru \& Muldner, 2009).

\subsection{Evaluation}

A pilot randomized controlled trial was conducted to examine the usefulness of the two supports proposed. In the study, participants received one of the following three interventions in their attempt to improve sleep quality: (1) sleep hygiene education alone (SH condition), (2) sleep hygiene education plus self-experimentation for behavior change tutorial (SH-SBT condition), and (3) sleep hygiene education, selfexperimentation for behavior change tutorial, and GaLLaG (SH-SBT-GaLLaG 
condition). After 1-week baseline data collection, participants created their initial behavior change plans for self-identified sleep-related issues, and tested the plans for two weeks. Then, they revised their current plans two times, and each revision was followed by a two-week test. The hypothesis is:

Individuals who created behavior change plans with the proposed tools (SH-SBT and SH-SBT-GaLLaG) are expected to have greater sleep quality improvement compared to the sleep hygiene control (SH) over seven weeks.

Although quantitative analysis on sleep quality improvement did not find significant results validating the hypothesis, distinctive aspects in each group's behavior change plans were observed through qualitative analysis. Difficulties encountered by participants were also discovered.

\subsection{Summary and Next Steps}

In this chapter, a goal of the dissertation study is described. Initially, people's prevalent failure in behavior change despite the significant impact of sustaining desired behavior is the focus. Although the HCI community has proposed various approaches to respond to this issue, such as sensor-based self-monitoring, gamification, coaching systems, etc., it is problematic that no or little attention has been given to support for individual creation of behavior change plans. Considering differences between individuals and the difficulty of meeting such diversity with pre-defined solutions, the aim of the present research is to support users' self-experimentation of behavior change plans. This chapter also introduces two levels of supports proposed to achieve this aim, (1) support of users' behavioral plan creation based on behavior change techniques, and (2) support of users' implementation of context-aware just-in-time interventions. The comparative study conducted to evaluate these approaches is also briefly described. 
Chapters three and four present detailed descriptions of how the proposed two approaches were developed. The methods and results of the comparative study are described in chapter five. Prior to these chapters, chapter two provides a literature review and theoretical foundations for explaining purposive behavior and failures and existing practices of self-experimentation driven by personal issues. Established ideas that informed the development of the proposed two supports are also presented. 


\section{BACKGROUND AND RELATED WORK}

\subsection{Self-Regulated Behavior}

In this section, literature that provides a theoretical foundation in understanding behavior change is reviewed.

\section{Social Cognitive Theory and Purposive Action}

A central feature of Social Cognitive Theory, which is a well established and often used psychological theory, is the recognition of the distinctive human capacity to exercise control over one's own thought process, motivation, and action (Bandura, 1986). Bandura advanced a view of human functioning that ascribes a central role to cognitive, self-regulatory, and self-reflective processes in human adaptation and change. These three facets have been the foundation for a wide range of behavioral interventions, particularly variants of cognitive behavioral therapy for numerous conditions including mental health concerns such as depression (Fava, Rafanelli, Grandi, Conti, \& Belluardo, 1998) and anxiety (Kendall \& Southam-Gerow, 1996)) and physical health problems such as binge eating (Grilo \& Masheb, 2005) and insomnia (Edinger, Wohlgemuth, Radtke, Marsh, \& Quillian, 2001).

Social cognitive theory views people as self-organizing, proactive, self-reflecting, and self-regulating rather than as reactive organisms shaped and influenced by environmental forces or driven by concealed inner impulses (Bandura, 2001). According to Bandura, many human behaviors (and largely unique to humans) are directed toward specific purposes, which are often identified using a variety of terms such as 'standards', 'goals', and 'intentions'. Bandura (1991) emphasized that: 
people motivate themselves and guide their actions anticipatorily by exercising forethought. They anticipate likely outcomes of prospective actions, they set goals for themselves, and they plan courses of action designed to realize values futures. Future events cannot be causes of current motivation or action, but by cognitive representation in the present, conceived future events are converted into current motivators and regulators of behavior.

Social Cognitive Theory incorporates previous concepts of operant conditioning and classical conditioning, which describes how external stimuli and rewards influence the increased or decreased likelihood of a behavior occurring in the future regardless of intention (Fisher, Piazza, \& Roane, 2011) via the concept of reciprocal determinism. Reciprocal determinism defines the dynamic interplay between personal (i.e., cognitions, beliefs), behavioral, and environmental factors and suggests that they eventually cointeract. For example, as a person increases their confidence in their ability to engage in a behavior (a concept called self-efficacy within Social Cognitive Theory), this increases the likelihood that they will engage in said behavior. Increasingly engaging in the behavior can then result in a person changing their context in some way (e.g., putting out running shoes if the action is running). This behavior then increases their confidence and the cycle of reciprocal determinism continues.

This theoretical perspective is foundational to the current dissertation, which actively seeks to emphasize purposive behaviors while also empowering individuals with tools and resources to both think creatively of behavior change strategies that they could engage in to achieve their goals (thus influencing the cognitions) while also empowering them with sensor and feedback systems that provides them greater control over their context for further supporting their behavior change goals. 


\section{Behavior Change Capability}

As discussed in chapter one, it is common for individuals to often engage in a goaldirected behavior for a short period but ultimately fail in transforming the behavior into a sustained behavior (Franz et al., 2007). Conceptually, these failures can be linked to a variety of factors from cognitive, affective, and other personal factors to social and technological influences. For example, Baumeister and his colleagues (1994) highlighted three causes of “under-regulation" (i.e., not sustaining a goal-directed behavior): (1) deficient standards (i.e., under-specified goals), (2) inadequate monitoring, and (3) inadequate ego strength or willpower. Similarly, the following skills that Zimmerman (2002) identified as important for supporting sustained goal-directed behaviors indicate possible causes of failure: (1) set specific proximal goals for oneself, (2) adopt strategies for attaining the goals, (3) monitor one's performance selectively for signs of progress, (4) restructure one's physical and social context to make it compatible with one's goals, (5) manage one's time use efficiently, (6) self-evaluate one's methods, (7) attribute causation to results (find out causes of outcomes, e.g., attribute weight gain to increased sedentariness this week due to a project deadline), and (8) adapt methods based on selfevaluation or self-judgment on causation of results. As delineated by those proposals, there is a wide range of factors that may lead to individual differences in successfully engaging in goal-directed behavior (Rosenbaum, 1980). In other words, behavior change can be improved by enhancing those aspects.

\subsection{Self-Experimentation for Behavior Change}

This section reviews existing practices of self-experimentation that can be divided into two parts, exercises motivated by scientific inquiries and work driven by personal issues. 
Self-Experimentation with Scientific Interests

The concept of self-experimentation arose from a few physicians who conducted experiments in which they studied themselves. This approach is in contrast to conventional research in which the experimenter studies other people or animals (Roberts \& Neuringer, 1998). The earliest recorded self-experiment is the work of Santorio, a seventeenth-century physician from Padua (Roberts \& Neuringer, 1998). For thirty years, he weighed himself before and after meals, the food he ate, and his excrements in an attempt to account for the differences in weights, which led him to suggest the existence of insensible perspiration (Castiglioni, 1931).

Since Santorio's research, many physicians have conducted self-experiments due to their interest in the causes and treatment of disease. Likewise, there have been many examples of self-experimentation in the early history of psychology (Neuringer, 1981). For example, Dressler explored his own response rates of his tapping on a Morse-code key as a function of time of day and of physical and mental exercise. He found that the response rate was related, as depicted by U-shaped curve, to the time of day. That is, response rate decreased with prior physical exercise and increased after prior vigorous mental exercise (Dresslar, 1892).

\section{Self-experimentation with Personal Problems}

Contrasted with the above examples in which self-experimentation was motivated by scientific interest (Roberts \& Neuringer, 1998), Richard Bernstein's work is an example where self-experimentation was driven by personal issues. In 1969, Richard Bernstein an engineer with diabetes - started to measure his blood glucose several times per day. He discovered that it varied widely over a day even though he was carefully following his 
doctor's recommendations. In diabetes, both high and low glucose have negative health effects. To reduce the variation of blood glucose, he began to conduct simple experiments. He discovered that many small doses of insulin spread out over the day (similar to how the pancreas functions for non-diabetics) maintained more stable glucose levels than one large daily dose of insulin, which was the typical prescription at the time. Lack of professional standing made it difficult for him to publicize his results, but he persisted and eventually his ideas spread and became accepted.

The QS movement is another example of self-experimentation driven by personal issues. QSers explore personal questions about themselves with methods they have chosen or designed (Choe et al., 2014). They diligently track many types of data and some share their best practices and mistakes through Meetup talks, blogging, and conferences (Choe et al., 2014). Their interests span from their physical or psychological health to productivity and social relationships. Although the movement's slogan "Self-knowledge through numbers", created by Wired Magazine editors Gary Wolf and Kevin Kelly, provides the notion that their goals are to know themselves, analysis investigating QSers' reports on their practices reveals that the ultimate aspiration of the majority was often to improve health and other aspects of daily life such as tracking weight to reach a target weight, logging sleep and exercise to return from an erratic lifestyle, and tracking time use to be more productive (Choe et al., 2014; Li et al., 2013). Thus, their experimentation results often identify behaviors that should be attained. However, as discussed in chapter one, it seems necessary to extend the existing self-experimentation by QSers from only discovering behaviors to obtaining plans that can facilitate individuals' fulfillment of the behaviors. 
Karkar et al. (2015) proposed a framework to support scientific rigor in selfexperimentation. In the framework, users obtain self-knowledge by undergoing three phases: formulating a hypothesis with knowledge on what may affect an issue of interest, testing the hypothesis, and examining the results of the test to target a behavior most appropriate in addressing the issue. Similarly, users may be able to obtain a behavior change plan that fits them better and is more effective in leading to improvement in target issues by generating a provisional plan, testing it, and examining the results of the test to identify facets that are suitable and effective.

\subsection{Support for Creation of Behavior Change Plans}

In the general process of self-experimentation for behavior change that the present research identifies based on the framework proposed by Karkar et al. (2015), particular attention is paid to the generating plan ideas phase and how to facilitate easy acquisition of potentially effective plans is considered. In addressing such issue, the rational problem-solving paradigm as a plausibly useful approach, given its application in various domains including in addressing social issues, was conceived. Second, incorporation of behavior change techniques was believed to be beneficial, as they are used as components of behavior change interventions (created by researchers or clinicians)(Michie et al., 2011a). In this section, characteristics of the rational problemsolving paradigm and problem-solving therapy, an application of the rational problemsolving paradigm in resolving people' social issues, are reviewed. Then, existing practices in which behavior change techniques are integrated in creating behavior change interventions are reviewed. 


\section{Rational Problem-solving Approach}

Newell and Simon's human problem solving theory was first formulated in the early 1970s (Newell \& Simon, 1972). Despite significant changes in the conceptualization since then (Dorst, 2003), it is still a dominant paradigm and many general facets of it are still used today in various fields such as engineering design (Ertas \& Jones, 1996), policymaking (Porter \& Ronit, 2006), and individuals' developing solutions for emotional issues (D'Zurilla \& Goldfried, 1971). In understanding its important facets, the following four aspects were noticed. First, in the rational problem-solving paradigm offered a general process, and Couger (1996)'s process developed in parallel to this paradigm for business and management decision-making is a typical example that reveals the fundamental idea of the general problem-solving process. The process consists of the following steps, opportunity delineation / problem definition, compiling relevant information / fact finding, generating ideas, evaluating and prioritizing ideas, and developing an implementation plan.

Second, problem definition is often considered the most important in obtaining solutions effective in overcoming the situation (Spradlin, 2012). Generally, processes start with understanding a given situation and defining a problem. This aspect emphasizes establishing a concrete problem (objective to achieve).

Third, once a problem is defined, it is often dissected into sub-parts as a means to handle the complexity of a problem. Then, answers for each part are generated and a combination generates a large number of possible answers to the original question in its entirety (Liikkanen \& Perttula, 2009; Powell \& Baker, 2008). For example, design of a document copier can be broken down into design of document handler, a printing device, and an image capture device. 
Fourth, the rational problem solving perspective involves the use of heuristics for the effective generation of alternatives. Heuristics are rule-of-thumb strategies that may or may not work in certain situations (Artinger, Petersen, Gigerenzer, \& Weibler, 2015). For example, in the most basic form with mere verbal statements, heuristics in management could include "start small, grow big organically" or "minimize initial investments" (Manimala, 1992). Heuristics are not algorithms that always produce a correct solution through a step-by-step procedure, and although they do not guarantee that the best solution will be achieved they serve as "quick and dirty" (easier) methods that lead to acceptable solutions (Yilmaz \& Seifert, 2011).

Originally, this problem solving approach was invented for problems that have a right answer obtainable by applying an appropriate algorithm (Newell \& Simon, 1972). That is, the work initially targeted so-called well-structured problems (e.g., laboratory problems such as anagram tasks or hypothetical impersonal challenges such as physics problems). However, its general concepts were also determined as adequate for handling relatively ill-structured problems that do not have a right answer and clear solution finding path, for example, how to increase water supply for a growing community. Most real-life problems fall into this category (Sinnott, 1989; Voss \& Post, 1988), and much more complicated processes should be engaged (Reitman, 1965).

\section{Real-life Personal Problem Solving}

Problem-solving therapy, originally introduced by D’Zurilla and Goldfried (1971), is an example of how the problem-solving paradigm has been adopted to help people resolve psychological and somatic issues. The approach is a type of cognitive behavioral therapy employed for a wide range of adjustment problems and disorders including depression, stress and anxiety disorders, weight problems, and other medical problems (A. Nezu, C. 
Nezu, \& D'Zurilla, 2012). In treatment, the patient is trained to use the problem-solving method to overcome their problems.

According to D'Zurilla and Goldfried (1971), the term "problem" refers to a specific "situation" or "set of related situations" to which a person responds to function effectively in their environment. The approach involves a series of sequential stages, and contrasted with the tradition of psychotherapy whereby a clinician is the agent for supporting change in a patient, the clinician supports patients to work through the stages to determine and implement a solution selected by the patient for a targeted problem (Pierce, 2012). Although there are many variants, the following five general stages represent a consensus viewpoint (D'Zurilla \& Goldfried, 1971):

1. General orientation (i.e., 'set' and attitudinal factors)

2. Problem definition and formulation

3. Generation of alternatives

4. Decision making (i.e., evaluation and selection)

5. Implementation and verification (i.e., assessment of outcome)

Problem orientation is concerned with how one views their ability to cope with a problem, whereas problem definition informs the specific and concrete definition of the problem and setting achievable and definable goals. The third step, generation of alternative solutions, involves creating various methods for solving problems and meeting personal goals while withholding judgment on their effectiveness. The fourth step, decision making, uses a systematic process to select the optimal solution for a problem from the generated list. Finally, solution implementation and evaluation involves the planning and initiation of solutions and subsequently evaluating the success of the solution. This approach intends that the rational problem solver, who "gathers 
facts and information about a problem carefully and systematically, identifies demands and obstacles, sets realistic problem-solving goals, generates a variety of possible solutions, anticipates the consequences of the different solutions, judges and compares the alternatives, chooses the "best" solution, implements that solution, and carefully monitors and evaluates the outcome (D’Zurill \& Nezu, 2010).

Effectiveness of problem-solving therapy has been validated by a number of outcome studies that evaluated its efficacy for a variety of different psychological, behavioral, and health disorders (D’Zurill \& Nezu, 2010). Problem-solving therapy indicates the adoptability and usefulness of the rational problem solving approach for individuals' development of behavior change plans.

\section{Behavior Change Techniques}

With the perspective of self-experimentation for behavior change targeted in this dissertation study, a fundamental issue under investigation is failure to carry out desired behaviors. In pursuing a solution to such problem, the question arose of what heuristics could help individuals generate solution ideas. Subsequently, the idea that existing behavior change techniques could be a useful resource was formulated.

Researchers in the psychological field have proposed and evaluated a wide range of behavior change techniques. For professional practitioners designing behavior change interventions, behavior change techniques are considered as active components (Michie, Abraham, Eccles, Francis, Hardeman, \& Johnston, 2011). The creation of behavior change interventions often involves combining various behavior change techniques into a multi-component intervention. Practitioners make selections and combinations of behavior change techniques based on their understanding of a problem and the desired 
solutions, which is information held within the end-user of an intervention. Based on the likely complexity of matching techniques to individuals, a type of "trial and error" approach seems inevitable for finding the "right" solution for each individual. The mechanism of technique delivery (e.g., prompts or reminders delivered by a person such as a friend or healthcare professional or by environments such as hand-washing signs) must also be taken into account as the method further impacts whether a technique will be used (Michie et al., 2011a). Importantly, selection of delivery mechanism is also driven by the idiosyncratic needs of the user.

With the perspective of the rational problem-solving paradigm, people can use those techniques as "heuristics" in the pursuit of solutions to their behavioral problems in the same way that professional practitioners use them. However, there are no algorithms to produce the right solutions for each individual. Thus, a trial-and-error approach is inevitable and self-experimentation is considered as a way to achieve this goal.

\subsection{End-User Creation of Context-Aware Applications}

With regard to the research aim of providing a tool that allows users' own construction of sensor-based feedback systems as just-in-time interventions, two existing studies are reviewed in this section. First, research on people's creative practice in everyday life, and second, provision of end-user development tools in HCI are reviewed.

\section{Everyday Creativity}

Although people are frequently characterized as consumers in modern society, creation of artifacts for everyday use is part of our culture, and Buechley (2009) described such phenomena as follows: 
People are driven to customize their objects and build things. Passionate makers sew dresses, build furniture, cook meals and write computer programs. People also spend copious amounts of time tinkering with the things they own. They decorate their notebooks, hack their cell phones and fix their cars. Groups often get together to share these techniques for building, modifying and embellishing artifacts, and vibrant social communities develop as a result.

In addition, Wakkary (2009) describes the ongoing presence of designers in the home, “everyday designers", based on his ethnographic study in which he observed people's spontaneous action of devising ways and objects to satisfy their everyday needs. Systems and routines continually evolve through design-in-use to address individual requirements and test-in-use that judges the quality and success of a designed system. Alexander (1964) suggested the presence of unselfconscious design as follows:

Unselfconscious process is a design process undertaken on a cultural level and over a long period of time, in which designed items are shaped gradually and continually to fit the surrounding, ever changing context. Individuals participate in this process in an unselfconscious way, simply recognizing a failure in the system and reacting in a corrective way to achieve a well-fitting form.

According to Wakkary \& Maestri (2007), people do not consciously understand the full complexity of the system, but have tacit understanding that is clear through use. People appropriate or create artifacts that can better serve them than ones developed by professionals, because they have tacit knowledge about their daily life that is ever changing. Such observation corresponds to tacit "knowing-in-action" proposed by Schön (1983) in his paradigm of reflective practice:

When we go about the spontaneous, intuitive performance of the actions of everyday life, we show ourselves to be knowledgeable in a special way. Often we cannot say what it is that we know... Our knowing is ordinarily tacit, implicit in our patterns of action and in our feel for the stuff with which we are dealing (p.49)

Alexander and Wakkary's arguments not only highlights individual involvement in design for everyday use, but also reveals the existence of a "bad fit” (Schön, 1983) between given systems and personal needs. Such a mismatch is considered as an 
inevitable result when the development of artifacts is separated from actual use of them. Developers create systems by making decisions for users regarding situational contexts and tasks that the designers can only anticipate (Fischer \& Scharff, 2000). However, anticipating all possible uses in advance is currently impossible (Greenbaum \& Kyng, 1991; Nardi, 1993) or at least costly (von Hippel, 2002). In addition, users' needs often evolve over time (Lieberman, Paternò, Klann, \& Wulf, 2006). Given such drawbacks, the users' continuous involvement in creation is considered as a prospective strategy to obtain systems that are more useful and usable to individuals.

In addition to the practical benefits, users' involvement in creation can contribute affective and educational values, which are well described by the following analogical statements by Resnick, et al. (1996):

The stereo has many attractions: it is easier than the piano to play, and it provides immediate access to a wide range of music. But "easy of use" should not be the only criterion. Playing the piano can be a much richer experience. By learning to play the piano, you can become a creator, not just a consumer, of music, expressing yourself musically in increasingly ever-more complex ways. As a result, you can develop a much deeper relationship with (and deeper understanding of) music.

\section{End-user Development Approach}

In the previous section, drawbacks of conventional development processes, which separate the design time from the use time (Fischer \& Scharff, 2000), were discussed. User requirements are diverse and prone to change and at times hard to identify precisely (Lieberman et al., 2006). End-User Development (EUD) in HCI is an approach that aims to resolve such issues by enabling users to develop and program information technology systems. The idea of EUD has been widely accepted across fields since the introduction of the first computers into common workplace use and represents the ultimate level of user participation in design processes (Syrjänen \& Kuutti, 2011). There 
can be two types of end-user involvement in creation, (1) parameterization or customization, and (2) program creation and modification. EUD involves more of the second set of activities because modification of software described in the first set is restricted to strictly predefined options or formats (Lieberman et al., 2006). Favoring the second form of activity, the approach aims to enable end-users who have not necessarily been taught how to write code in conventional programming languages to write programs that instruct computers to conduct the individual's desired objective (Cypher \& Halbert, 1993). However, approaches in the first set are often utilized for a "gentle slope" of increasing complexity to allow users to easily progress from the first to the second set of activities (Lieberman et al., 2006).

Although there are many tactics relevant to building "better" end-user development tools, task-specificity, commonly credited to Nardi (1993), is considered fundamental for development tools to be adoptable:

We have argued that people are adept at learning formal systems and notations. If that is true, why then, have so many trouble learning conventional programming languages? The answer is that it is only when people have 'a particular interest in something', such as knitting or baseball, or..., that they readily learn the formal languages and notations that describe the elements and relations of the system of interest.

This argument highlights the idea that end-user development tools should support a specific interest of people (i.e., application domain) and use formal languages and notations that closely match these interests and domain knowledge. Similarly, Fischer (1993) emphasizes representations of evolving artifacts that are less abstract and less alienated from practical use situations to support human problem-domain interaction. However, these task or domain specificity approaches inevitably sacrifice generality for the power of specialized interactions. Thus, careful study of those circumstances is necessary to achieve the right level of task-specificity (Nardi, 1993). 
So far, the significance of end-user development has been discussed and has addressed the desirability for people to be designers. However, this position does not postulate that being a consumer is wrong. Rather, Fischer and Scharff (2000) asserted the existence of a continuum of user roles ranging from a passive consumer to a 'meta-designer' and the need to provide appropriate support for each role.

\section{Creation of Context-aware Applications}

Considering that building appropriate context-aware applications requires substantial personal knowledge about users' activities and environments, the end-user development paradigm is beneficial in this area. Dey and his colleagues (Dey, Sohn, Streng, \& Kodama, 2006) highlighted the need that users have the ability to create and modify context-aware applications. The researchers asserted that without such ability, contextaware applications acting implicitly often annoy users and fail to meet their needs and ever-changing activities and environments. Embracing the end-user development paradigm, several tools for users' implementation of context-aware applications have been proposed and aim to enable users with no or limited programming and other technical skills to prototype applications incorporating sensors and actuators (Beckmann \& Dey, 2003; Dey et al., 2006; Chin, Callaghan, \& Clarke, 2006).

Although there has been considerable research investigating end-user programming tools for the creation of context-aware applications in home environments, most tools intend to support control of appliances or environmental equipment, and little attention has been paid to the provision of toolkits focused on behavior change. Focusing on this area will likely involve addressing user needs and situations that are distinct from the ones currently used by existing smart home control and automation systems (GarcíaHerranz, Haya, \& Alamán, 2010; Dey et al, 2006; Chin et al., 2006). For instance, ease of 
use is typically considered as the most prominent issue regarding user satisfaction (Chin et al., 2006) in developing tools for home control and automation systems. Shifting a focus from general home environment control to support for behavior change requires expanded evaluation criteria beyond ease of use, including effectiveness in fostering behavior change. Thus, functionality and research methodology needs to be advanced to address these requirements.

\subsection{Summary and Next Steps}

In this chapter, the theoretical basis related to people's behavior change is firstly reviewed, including the behavioral theory highlighting people's purposive goal-directed behaviors and multiple influential factors including cognitions, behavior, and environments. Literature on existing practices of self-experimentation was surveyed. Especially, existing research on the QS movement revealed an individual's interest and capability of engaging in a personal journey to obtain self-knowledge for their lifestyle improvement. However, a limitation in its current focus was observed, that is, no or little effort of facilitating attainment of behaviors found necessary seemed problematic. Therefore, the need of acquisition of behavior change plans that can facilitate their attainment was highlighted, and in addressing how the plan can be supported, the rational problem-solving paradigm was selected as plausibly useful. Problem-solving therapy that was developed based on the rational problem-solving paradigm confirms such a belief. The key characteristics of the rational problem-solving paradigm were studied, and the necessity of having heuristics in making behavior change plans was established. Behavior change techniques were considered as a promising resource that serves such heuristics, and existing use by professionals validated this assumption. 
Finally, the end-user development paradigm and existing tools for end-users' programming of context-aware applications were reviewed.

In the next chapter, the development of a tool for constructing context-aware applications as JIT interventions, one of the two approaches explored in this dissertation, is described. First, components of the tool including its overall architecture integrating each component and the rationale of the design decision in choosing them is presented. Second, a programming interface of the tool, which was developed to allow users with no programming skills to create applications using the tool, is described. Third, the user study conducted to investigate usability of the programming interface is presented. 


\section{TOOL FOR CREATION OF JUST-IN-TIME INTERVENTIONS}

\subsection{Approaches}

In developing a tool that allows users to construct context-aware applications realizing the just-in-time information strategy, the existing platform, GaLLaG was adopted. The Motivational Environments Research group directed by Dr. Winslow Burleson originally developed this tool to enable researchers to readily develop hybrid reality systems (Burleson et al., 2009). The platform enables rapid prototyping of rule-based systems that include a variety of sensing (from simple magnetic sensors to smart phone GPS tracking) and media event components (from text messages to mobile phones to sound play and robot movements). While the tool is used for diverse purposes such as tangible learning environments (Burleson et al., 2009) or a system for helping people with mild dementia dress themselves (Lozano, Burleson, Ravishankar, J. Lee, Muldner, \& Mahoney, 2014), the current research identifies a use for realizing plausible applications for behavior change.

In adopting existing GaLLaG, users' ability of augmenting their home environments is the focus of the present research. People's home may be where they spend more time than any other space. In addition, by having control and ownership, people "invest extraordinary amounts of time, money, and emotional energy to mold homes into living spaces that meet their needs" (Initlle, 2002) for purposes from relaxing to conducting business. With those assumptions, it was determined that the home is a logical place to investigate first. In addition, the aim is to develop a tool that is simple in terms of technological complexity but useful in embracing potential needs. To that end, several key facets are incorporated as described in the following section. 


\subsubsection{Rule-Based Systems}

The rule-based approach for inferring events in context-aware computing has been widely adopted due to its logical simplicity and effectiveness in controlling various situations (Truong, Huang, \& Abowd, 2004; Dey et al., 2006; García-Herranz et al., 2010; Dalal, Alwan, Seifrafi, Kell, \& Brown, 2005; Vurgun, Philipose, \& Pavel, 2007). For example, a rule for detecting meal preparation can be: "IF resident was in the kitchen AND (resident accessed meals ingredients cabinet AND resident accessed plates or utensils cabinet) OR resident used an appliance THEN a meal was prepared" (Dalal et al., 2005). In developing an in-home health status monitoring system, the Medical Automation Research Center (MARC) at the University of Virginia compared several data analysis techniques to infer the occurrence of activities based on spatial-temporal relations among sensor events, including clustering and mixture models and the rulebased approach. They adopted the rule-based approach favoring its simplicity, computational efficiency, and scalability (Dalal et al, 2005).

The rule-based approach provides a mechanism for distilling relatively complex concepts into pragmatic decisions that an end-user programmer would feasibly engage with. Dey and colleagues (2006) collected 371 application descriptions during a user study in which participants were provided with a description of a smart home, a concept that most were already familiar with, and asked to (1) describe how, when, and where they would want music to play in their smart home and then (2) describe scenarios open to any purposes that they found useful and desirable in their home. By analyzing the collected descriptions, the researchers found that every subject described their application in terms of if-then rules, using the form "if I..." or "when I..." am in a particular situation, "perform this action". For example, "The nightlight in the bathroom 
should dim at night.” This result implies users' familiarity with rule description, and given this finding, users' rule-based definition of a situation seems plausible.

For better inference accuracy, more sensors should be included in the home. For example, if someone sits on a dining chair, it does not necessarily mean that they are eating a meal. To address this issue, an additional sensor can be attached to a utensils drawer. However, such addition increases cost in terms of sensor installation and management and rule making (Dalal et al, 2005). While high accuracy may be important for better user experience, this end-user development context may reduce users feeling disturbed by wrong triggers. Through creating rules, users can be familiar with potential limitations and such understandable errors can be expected to result in less overall annoyance.

Overall, considering the versatility of rule-based programming, ease in user understanding, and plausible tolerance of its limitation, this approach is considered appropriate for end-user development of just-in-time information applications.

\subsubsection{Sensors}

To develop the rule-based inference described in section 3.1.1, the construction tool uses wireless state-change sensors for detecting use of objects and user location. Simple statechange can often provide credible clues about activity (Dalal et al., 2005; Dey et al., 2006). For instance, a magnetic sensor attached to the garage door sensor can strongly suggest a user is coming home. Use of multiple sensors can increase accuracy of recognition of a variety of activity types (Tapia, Intille, \& Larson, 2004). In addition, this approach can mitigate users' concerns of privacy because the system does not collect 
personally identifiable information (e.g., facial or body images or voice recordings) (Tapia et al., 2004).

\subsubsection{Responses}

The tool allows three prompting methods, (1) audio content via wireless speakers, (2) text messages via mobile phones, and (3) appliance control. Audio prompts can include machine speech of user-inputted text or playback of user-added sound files (music and sound effects). Audio has an advantage in that it can often capture a person's attention, if they are in the vicinity and the space is quiet enough, regardless of where they are currently looking, which is required for catching attention via a video display. In addition, sound, especially music, is well known to readily induce emotions (Konečni, 2008), which is considered as one of crucial factors that influence users' self-regulatory behavior (Muraven \& Baumeister, 2000) and a persuasive medium (Wilson, 2003). However, audio prompts are limited by the need for individuals to be near the speakers. Meanwhile, people tend to keep mobile devices near them even within their homes (Oksman \& Rautiainen, 2003). As such, the use of text messages is considered useful as a complementary prompting strategy. Lastly, by using the appliance control component of the tool users can make appliances (e.g., lamps) turn on or off as a prompt.

\subsection{Architecture and Applications}

To implement the approaches presented previously, the GaLLaG tool was equipped with off-the-shelf hardware and software technologies. For sensing, the system incorporates passive infrared motion sensors and magnetic sensors that use the X10 wireless protocol (http://www.x10.com)(Figure 1), and Insteon on/off modules (http://www.insteon.com/technology/). X10 door sensors and motion sensors can be 
transformed for situations that the original forms cannot satisfy. For instance, for a mat to detect a user sitting on a chair, two sides of the mat are separated with a flexible material and each contact of a door sensor was attached to one of the two sides (Figure 2). To detect presence of a person in a small target area, for example, a user lying on the bed, a passive infrared motion sensor was attached at the end of a long tube (Figure 3).
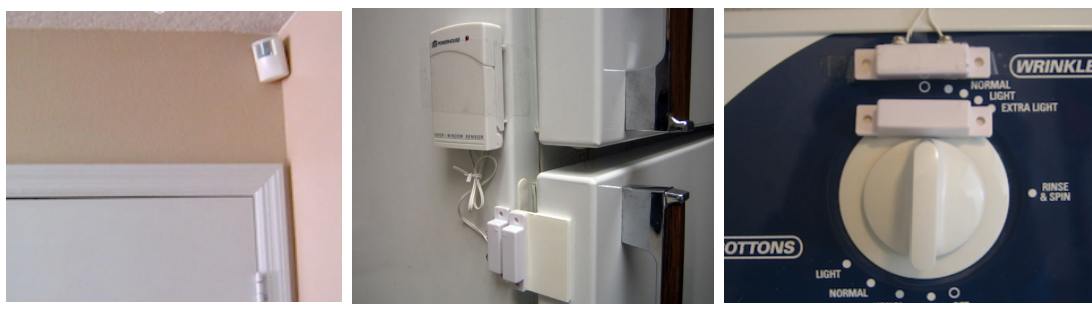

Figure 1. Sensor Use Examples: (from left to right) X10 motion sensor to detect users' entering a space (e.g., a room), or taking a book, X10 door sensor to detect users' opening the refrigerator, and X10 door sensor to detect the start or end of the laundry cycle.
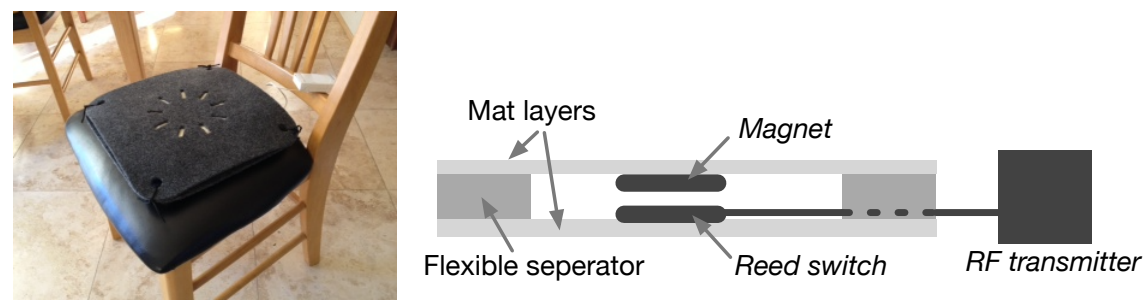

Figure 2. Example of X10 Door Sensor Transformation

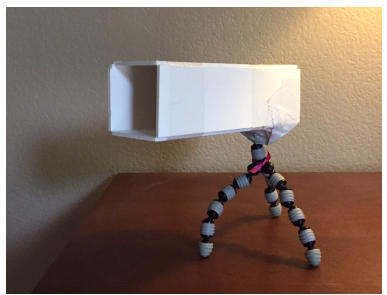

Figure 3. Example of X10 Motion Sensor Transformation

For sound play, the tool adopts Apple's wireless audio streaming technology, Airplay

(http://www.apple.com/). The central part of the tool is a Mac computer

(http://www.apple.com/) that runs Indigo home automation software 
(http://www.indigodomo.com/), receives data from X10 and Insteon sensors, builds inhome WiFi network with Apple's wireless routers, Airport Express for sound play, and connects to the Internet network (Figure 4).

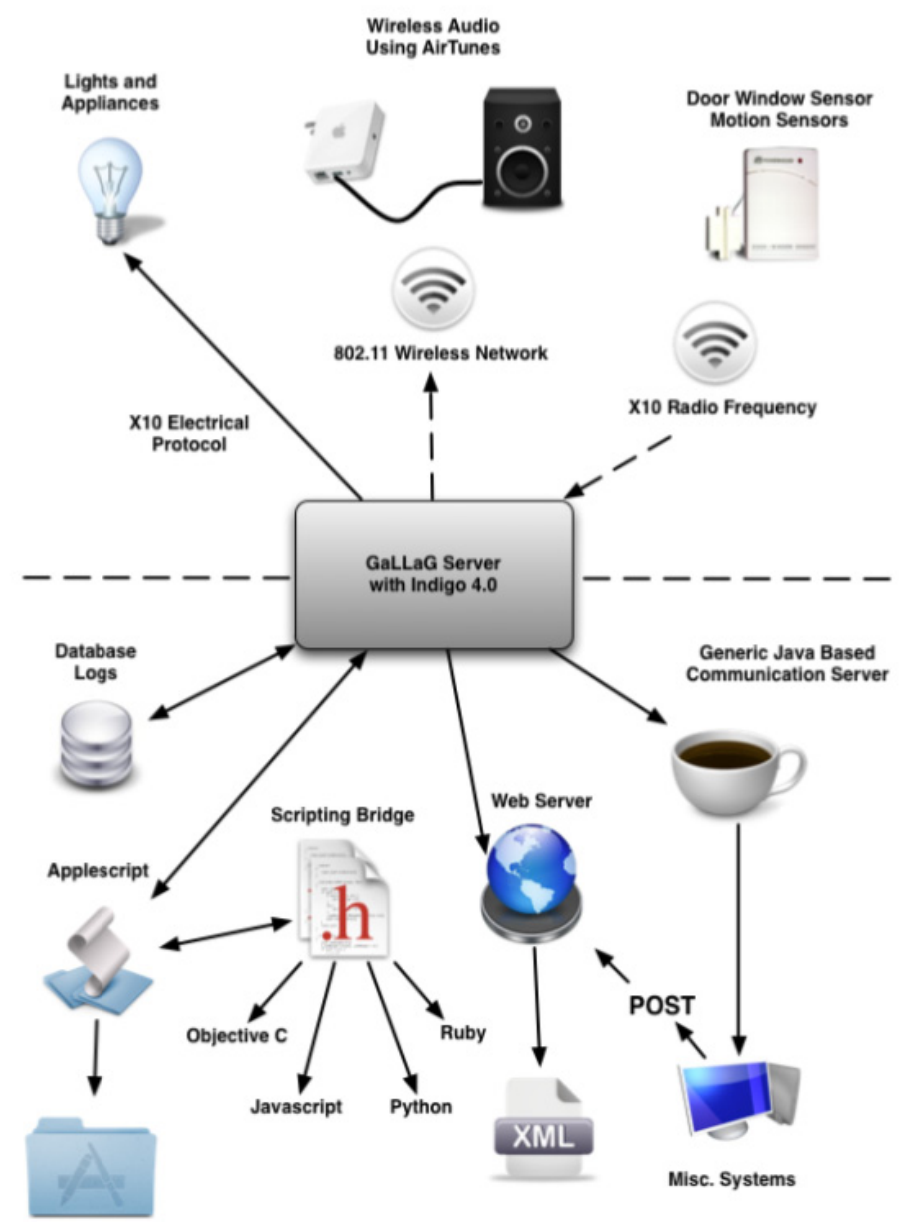

Figure 4. GaLLaG Construction Tool Architecture

The Indigo home automation software collects the sensor-generated data, runs application scripts, and produces outputs that includes playing sound via a speaker connected to an Airport Express, sending emails to cell phone carriers for text messages, and turning appliances on or off. The system can also store data in SQLite database (http://www.sqlite.org/) and developers create applications by writing codes of AppleScript, a scripting language created by Apple Inc. (http://www.apple.com). For 
easy use of related applications such as iTunes (http://www.apple.com/itunes/) for playing music and SQLite for managing data, the Motivational Environments Research group developed a set of AppleScript functions.

With the GaLLaG tool, a variety of applications varying in complexity can be built. Here, several examples are introduced. First, imagine a user who wants to watch less TV and read more books instead. To support this behavior change goal, the following system could be implemented: "the application senses when the TV is turned on and triggers an audio cue prompting them to turn off the TV and read a book." Furthermore, the system could be extended such as: "(after the audio cue) when the application senses the TV is turned off AND the user takes out a book then the system turns on the lamp that the user normally uses when reading". While these examples are largely simple and linear, the tool also allows developers to build more complex patterns such as (J. Lee, Walker, Burleson, \& Hekler, 2014b):

- Detection of an action based on duration of engaging in a behavior, for example, "After brushing my teeth for 2 minutes, play applause from the wireless speaker in the bathroom"

- Compounded actions being examined only after a previous action occurred, for example, "If I have not washed my hands in 10 minutes after coming home, a 'water' sound clip plays"

- Actions to engage in within pre-specified time-periods, for example, "If I brushed my teeth at three consecutive nights, my favorite songs play when I open my chocolate box" 
- The use of delayed sensing of subsequent actions, for example, "Two minutes after an entrance door is closed, I hear music from the bathroom inviting me to wash my hands"

\subsection{Development of a Programming Interface}

The hardware and software architecture of the GaLLaG tool allows users to create GaLLaG applications by writing AppleScript codes. Although the tool enables rapid development integrating a variety of technologies, it still requires some degree of programming skills, which limits use by people without programming skills. Thus, intending to lower such barrier so people who have no or limited programming skills can create GaLLaG applications easily, Luis Garduño and the Motivational Environments research team developed a visual programming interface, GaLLaG Strip³. This work was inspired by previous systems that use a simplified menu-based or metaphor-based Graphical User Interface (GUI) (Dey et al., 2006; Humble \& Crabtree, 2003; Truong et al., 2004) to allow end-users to specify applications visually without requiring them to write any code (Dey et al., 2006). Furthermore, the development of GaLLaG Strip hypothesized benefits of mobility and tangibility in end-user programming for contextaware applications (J. Lee, Garduño, Walker, \& Burleson, 2013).

While most end-user programming interfaces for context-aware applications imply desktop computers as their usage environments, GaLLaG Strip assumes mobile platforms. Its smartphone-based user interface allows users to roam within a sensorinstrumented space while programming their applications. In their previous work, the team observed participants' use of environments while they brainstormed ideas for

3 The development was part of thesis fulfillment of Luis Garduño. 
context-aware applications. The team conceived the potential utility of placing users in a location to which a target behavior is related. This mobile approach is validated by values of contextual design, rapid prototyping, and in situ creation acknowledged in the HCI community (De Sá \& Carriço, 2009; Seifert, Pfleging, del Carmen Valderrama Bahamóndez, Hermes, Rukzio, \& Schmidt, 2011).

Going beyond mobility, GaLLaG Strip is tangible, in that its interface enables programming by a physical demonstration of envisioned interactions with the same sensors and objects that users later encounter in their finished application. Users manipulate objects that are part of their daily lives rather than models (e.g., abstract blocks or miniatures) that most tangible interface systems have adopted (Horn, Solovey, Crouser, \& Jacob, 2009; Beckmann \& Dey, 2003). Therefore, the approach of GaLLaG Strip can be referred to as real-world tangibility. It may be frequently subtle for a person to identify the contextual cues that trigger their behaviors, especially habits that occur in an automatic way (Wood \& Neal, 2007). Therefore, the current research assumes that users may be better reminded by performing actions.

In GaLLaG Strip, users define their programs in a linear fashion by using simple if-then conditions, that is, it does not allow nested loops or conditionals. In developing a tool as an attempt to support users' experimentation for behavior change, it seemed appropriate to start with simple but essential programming logic. In early field studies conducted by the team, it was demonstrated that participants frequently generated application scenarios that only involved simple if-then rules (Dey et al., 2006). For example, "it plays a 2-3 minute song every time we walk by the dishwasher, with the intent of suggesting we clean just until the song ends." 


\subsubsection{Related Work}

Visual Programming Tools for End-user Creation of Context-aware Applications

There has been considerable research to enable users with little or no technical expertise to prototype context-aware applications. The majority of these efforts have employed visual programming methods (Dey et al., 2006; Humble \& Crabtree, 2004; Truong et al., 2004) by using either metaphor-based GUIs or simple input. One such tool was developed by Humble and Crabtree (2004), which is a GUI based on the "jigsaw puzzle" metaphor. They allowed users to connect digital jigsaw puzzle-like components that represented sensors and devices in various left-to-right combinations to form expressions. Humble and Crabtree believed that although their linear programming model constrained users in terms of expression possibilities, it allowed for easy reconfiguration and helped users to have a better sense of the information flow. Similarly, Truong et al. (2004) employed a GUI based in a magnetic poetry metaphor for $C A M P$, allowing users to define context-aware applications through the arrangement of fridge magnet-like words. With a more traditional PC-based GUI in iCAP, Dey et al. (2006) enabled users to create context-aware applications by selecting menus and dragging and dropping graphical elements such as objects, activities, locations, people, and time.

\section{Tangible Programming Tools}

Several studies have developed tangible tools for context-aware programming. For example, SiteView by Beckmann and Dey (2003) allowed users to build rule-based applications for home automation through tangible interaction with physical objects placed on a small-scale floor plan. Their system used RFID and a top-mounted camera to 
capture the rules that users wanted to program and an environmental display to show images of how the real environment (represented by the floor plan) would look when the rules were applied. Beckmann and Dey described the intuitiveness of their interface, which lowered the programming difficulty for novice users.

While use of models is dominant in tangible interface systems, some researchers have explored the use of real environments for context-aware programming. Chin et al. (2006) proposed PiP (Pervasive interactive Programming), an if-then rule system that allows users to show the behaviors they intend to program through physical interaction with a sensed environment. The programming interface in PiP is composed of several networked devices (e.g., lamp, phone, fridge) and a PC-based GUI called PiPView. Users have the choice to program their applications solely through physical demonstration, in addition to the GUI, or a combination of both. The researchers reported that majority of participants (72\%) preferred to program through physical interactions. Likewise, the HomeMaestro project by Salzberg (http://shaunsalzberg.com/medialab/homemaestro) allows users to move about a sensed environment and interact with physical artifacts to program context-aware applications using a mobile phone. GaLLaG Strip is parallel with these systems as all three employ real-world tangibility as a primary interface method, focusing on the potential benefits of its intuitive quality.

During the informal interviews conducted in the present research with six users who had experience making applications using the GaLLaG tool, respondents were asked about their thought process when developing application ideas. The users said that they typically imagined a particular location in their house (e.g., kitchen, living room) or looked around the space where they were currently located. Another common process was thinking about what they usually do during a particular period (e.g., morning, 
evening), that is, they mentally placed themselves in situations of everyday life.

Additionally, it was observed that interacting with physical objects served as a cue to

remind users of situations that they wanted to address. Based on these findings, taking a mobile and tangible approach to knit application creation closely with users' environment and behaviors was considered advantageous.

\subsubsection{User Experience and Interface Design}

In this section, the process of creating a new GaLLaG application using GaLLaG Strip, its structure, and the customization options available is explained.

\section{Example Application}

As a sample application, imagine that a user wants to create an application that senses when the TV is turned on and triggers an audio cue to remind the user that reading would be a better behavior than watching TV. When GaLLaG Strip starts, the user can see the list of applications they created previously (Figure 5) and then enable and disable them according to their needs. To add a new application, the user touches the 'plus' button and the demonstration screen is presented. The demonstration screen is where users demonstrate what they want to program. The screen has a recording mode and an edit mode. When creating a new application, the demonstration screen moves directly into recording mode, where the system listens for sensor events triggered by user actions. Following the previous example, the user turns on the TV (i.e., with the TV's remote control) and a frame with an icon of a TV turned on appears on the demonstration screen. Because that is the only event the user wants the application to listen to at the beginning, the user touches the pause button to stop the recording. When the user touches the pause button, the application goes into edit mode in which the 
current application can be reviewed and edited. Now, the user wants to add the audio cues to remind them that they should read instead, so the user touches the plus button to add an audio response, selects the sound to play, and adds another response to make the system speak (i.e., text to speech) the phrase: "You should read instead of watching TV". At this point, the user has added two response frames and has three frames in the application, one action frame (TV turned on) and two response frames (audio cues). Next, the user decides to make the application sense when they turn the TV off and provide a reward with an achievement sound cue. To achieve this, the user touches the record button and the demonstration screen moves to recording mode again, the user turns off the TV using the TV's remote control and an action frame, with an icon of a TV turned off, is appended in the application (Figure 6). The final frame the user wants to add is an achievement sound as a reward for turning off the TV, so the user touches the pause button to switch to edit mode, touches the plus button to add a response, and selects the achievement sound (Figure 7).
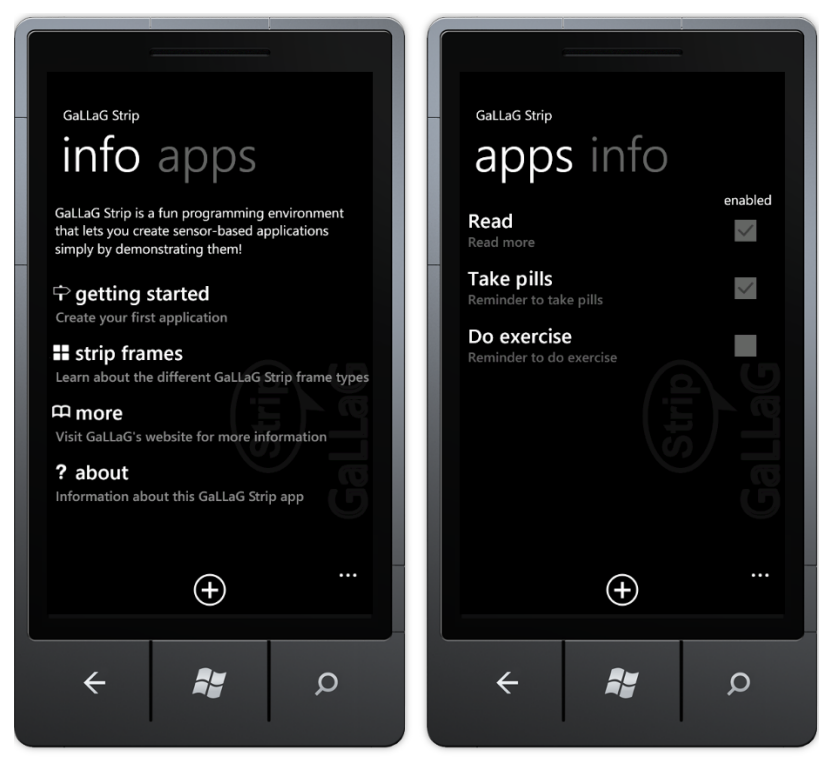

Figure 5. Main and Application List Screens of GaLLaG Strip 


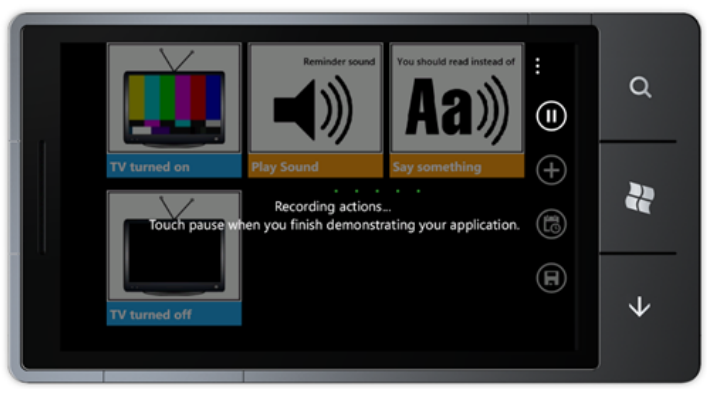

Figure 6. Adding an Action Frame in Recording Mode

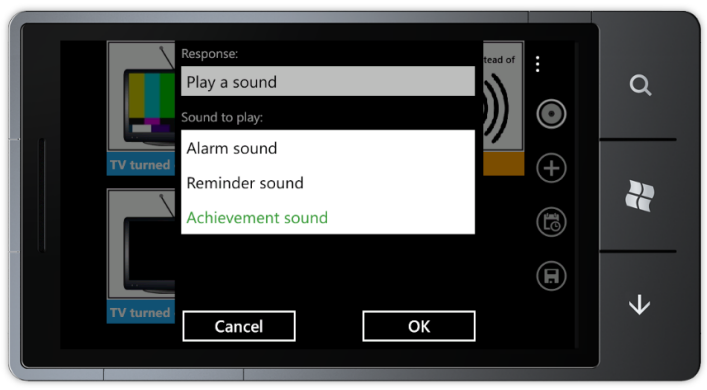

Figure 7. Adding a Response Frame

When the user finishes creating the application, they touch the save button and the system configures itself to do what the user just programmed. After the application has been configured in the server, it is ready to be run and can be tested simply by interacting with the sensed object (i.e., the TV) and performing actions previously defined in the application.

\section{Types of Frames}

In designing the graphic user interface, a comic strip metaphor, inspired by the work of Modugno and colleagues in Pursuit (Modugno, Corbett, \& Myers, 1997), was employed. In the GUI, a GaLLaG application is represented through a sequence of frames, “application strip", and three types of frames (action, response, and time-date) are provided. Action frames represent the user's actions within the sensed space and are 
shown as blue frames in the application strip. These frames have a default text label and image depending on the type of sensor (see Figure 8).

Time-date frames are conditions set by the user and they constrain the application's execution to a particular time, date, or both. These frames are shown in green and display the selected date or time as their text label. Additionally, time frames have a parameter to display the selected days of the week (see Figure 9). Time and date frames can be combined to create conditions based on both a date and a time, that is, an application can have up to two date and time frames.

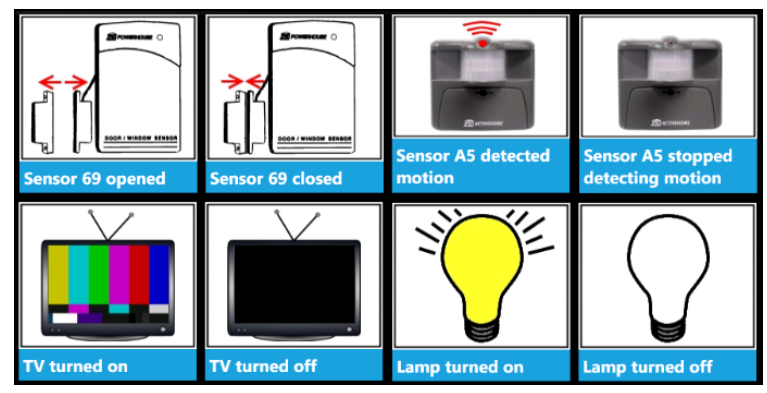

Figure 8. Action Frames

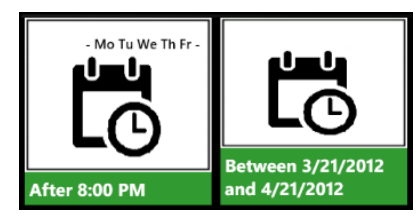

Figure 9. Time and Date Frames

Response frames represent actions that the system performs and are set by the user (Figure 10). This type of frame is displayed in orange and has a text label and image related to the type of response selected. Response frames can also have an additional parameter that is displayed in text above the frame's image. 


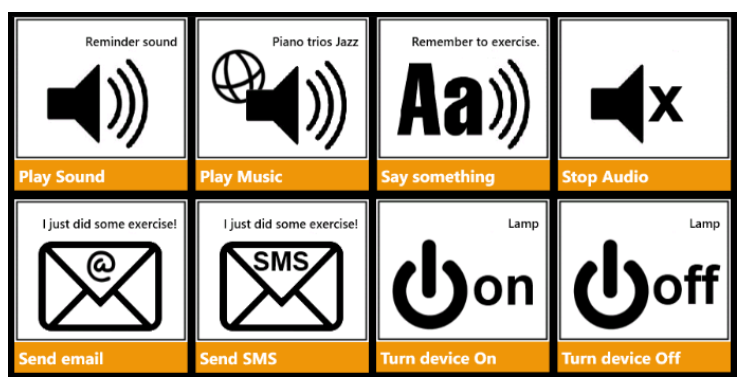

Figure 10. Response Frames

Action frames are initially displayed with a default text label and image depending on the sensor being activated. For easier recognition, users can customize the label by changing the text and taking a picture with the phone's built-in camera. Figure 11 provides an example of a frame represented by a captured book image and user-typed label, which was changed from the default motion sensor image and text.

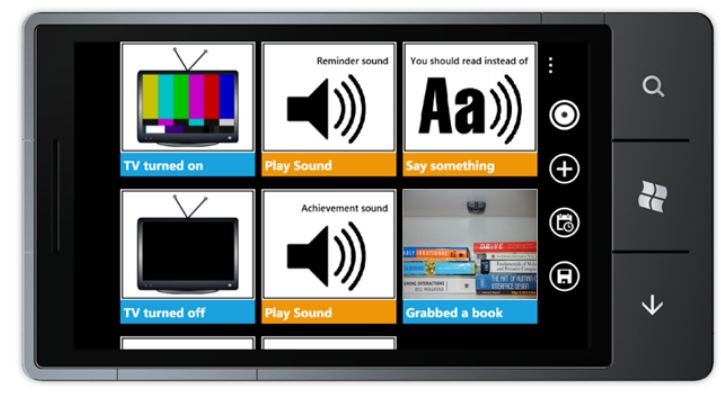

Figure 11. Action Frame after Being Customized

\section{Application Strip}

The programming model for GaLLaG Strip is a linear, if-then rule-based model and is read from left to right and top to bottom. Preceding actions or responses must occur in the same sequence as they appear before the current one can execute. Similarly, time and date condition frames need to evaluate to true for the rest of the application to execute. 
When reviewing or editing an application, users can scroll up and down the screen to see the whole application, as it may not be possible to see the entire application at once.

\subsection{User Study of the Programming Interface}

While there are a small number of studies that propose end-user programming tools integrating real-world tangibility (Chin et al., 2006; HomeMaestro project, http://shaunsalzberg.com/medialab/homemaestro), no substantial evaluation has been conducted to examine effects on the user's experience. Therefore, a controlled experiment was conducted in the present research that compares this approach with two other conditions (mobile non-tangible interface and non-mobile non-tangible interface), regarding ease of use, engagement, and the user's ideation experience (Horn et al., 2009; Xie, Antle, \& Motamedi, 2008). To explore the effects of real-world tangibility, three questions were formulated: (1) Does a combined mobile and tangible end-user programming environment make use of the programming tool more difficult? (2) Does it increase end-user engagement? (3) Does it facilitate ideation processes?

\subsubsection{Method}

The experiment compared three conditions: (1) mobile-tangible (MT), (2) mobile-menu (MM), and (3) stationary-menu (SM). A between-subject design was chosen primarily to avoid participants' potential bias to "please the experimenter." In all three conditions, participants were asked to complete the same tasks in the same laboratory setting as the

first study. MT participants programmed their applications, using the version of GaLLaG Strip described above, by physically interacting with the sensed environment.

For the user study, a space was arranged to resemble a living room with objects such as an armchair, a TV, books, etc. (Figure 12). A total of 15 sensors were placed around the 
space. For example, X10 magnetic sensors were placed on the laboratory's front door, the TV remote, a plant vase, two drawers, a digital portrait, and the air-conditioner's (AC) thermostat.

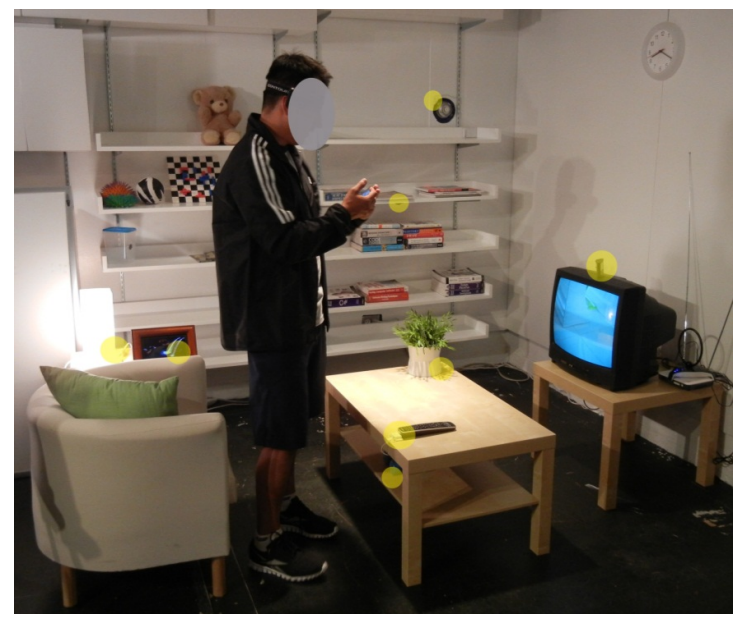

Figure 12. Living Room Setting Built for GaLLaG Strip User Study

Participants in the MM condition programmed their applications through an equivalent menu-based GUI on a mobile device. The menu-based GUI for the MM condition differed from the GUI of the MT condition in that actions were added to the application manually by selecting them from a list of available sensors and related sensing features (e.g., a motion sensor detecting motion or the ends of a magnetic sensor being separated, as illustrated in Figure 13).

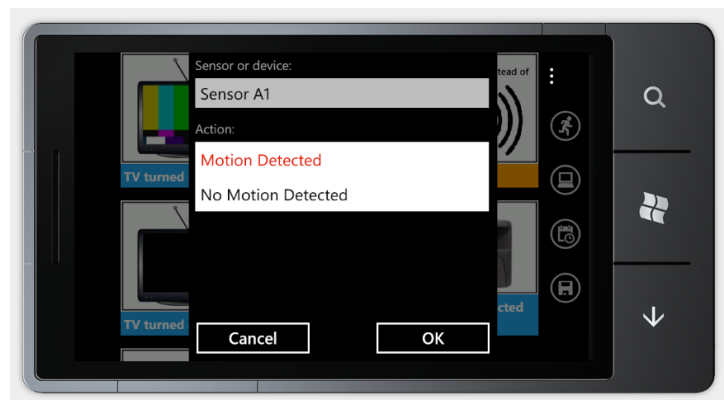

Figure 13. Manually Adding a Motion Detected Action 
With this new feature, users in the MM condition were able to create an application completely through the GUI, that is, without needing to physically demonstrate the application. In this mode, action frames and the desired sensors could all be added through menu options. Participants were given a list of the sensors with their IDs and a picture so they could locate them in the lab setting. However, users were not required to carry the list while programming. In both the MT and MM conditions, participants were able to move around the space while creating their applications.

Participants in the SM condition used the same menu-based GUI as the MM condition, but on a desktop computer through a Windows Phone emulator. Thus, they programmed their applications in a stationary manner. SM participants faced away from the living room but were able to turn their heads and look at the living room setting. Through these conditions, the goal was to isolate the effects of being able to move within an environment (as in the MM condition) from having the movement influence the developing program (as in the MT condition).

\section{Participants}

A total of 36 individuals were recruited through email lists and Craigslist. Seventeen were female and 19 were male. Ages ranged from 18 to 39 years of age. Participants were required to know the basics of how to use a computer and a smartphone. 19 participants (6 men, 13 women) had non-engineering backgrounds (e.g., dance, industrial design) and 17 (13 men, 4 women) had engineering backgrounds (e.g., chemical engineering, civil engineering, computer science). Participants were randomly assigned to one of the three experimental conditions (12 participants per condition). Five non-engineering and seven engineering background participants were in the MT condition, six non-engineers and 
six engineers were in the MM condition, and eight engineers and four non-engineers were in the SM condition.

\section{Procedure}

Participants joined one and a quarter-hour session individually. The session began with a video tutorial presenting primary components (sensors and responses available), sample application scenarios, and a demonstration showing how to program the sample application (which differed depending on the condition assigned to a participants). Then, the programming tool corresponding to their assigned condition (i.e., a mobile phone for the MT and MM conditions and a desktop computer for the SM condition) was provided to participants. The participants also received a printed list of the sensors available, showing a picture of their location and their assigned sensor ID.

Participants were asked to complete three tasks: simple, complex, and free-form. For the first two tasks, participants were provided textual description of interactions they had to program (Figure 14). For the simple application task, participants were asked to program an application with two actions and two responses, for example, "if you enter the living room and you turn the TV on, then make the system play the reminder sound and make the system say 'Remember to take your pills”'. In the complex application task, participants were asked to program an application with one time-date condition, three actions, and three responses, for example, "if the time is after nine in the morning and you turn on the AC and then open the front door, then make the system play the alarm sound, and make the system say 'Turn off the AC'”; if you then close the door (i.e., if you leave the house), then make the system send an SMS to your mobile phone with the message 'You left the AC on!'” For the free-form application, participants were asked to think about a personal scenario that they would like to program. They were given a blank 
to use", and "I would imagine that most people would learn to use this system very quickly”. To assess engagement, the following questions were asked: 'This experience was fun", "I felt creative when using the system", and "I think I was able to easily program a scenario idea that I came up with”. From the semi-structured interviews (based on participants' survey responses), further insights were gathered. Participantgenerated application ideas produced in the free-form task and after-session brainstorming were also collected.

\subsubsection{Results}

\section{Ease in Use}

A two-way ANOVA was performed with ease of use as the dependent variable and engineering background and condition as factors. While condition was not significantly related to ease of use $(F[2,30]=1.36, p=0.271)$, engineering background was significantly predictive of ease of use $(F[1,30]=5.41, p=0.027)$. Additionally, it was found that engineering background interacted with condition to predict ease of use $(F[2,30]=6.23, p=0.005$, see Table 1 for means $)$. Contrasts revealed that nonengineers found the $M T$ condition to be significantly more difficult than the $M M$ condition $(\mathrm{p}=0.006)$ and the $S M$ condition $(\mathrm{p}=0.003)$. For the $M T$ condition, nonengineers perceived the activity as significantly more difficult than the engineers did $(p<$ o.001). To explore possible gender effects, a two-way ANOVA was performed with ease of use as the dependent variable and gender and condition as factors. Gender did not have a significant effect on ease of use $(F[1,30]=2.63, p=0.155)$, and the interaction between gender and condition $(F[2,30]=1.27, p=0.297)$ was not significant. 
Table 1. Descriptive Statistics for Ease of Use and Engagement

\begin{tabular}{cccccc}
\hline \multirow{2}{*}{ Condition } & $\begin{array}{c}\text { Major (Number of } \\
\text { participants) }\end{array}$ & \multicolumn{2}{c}{ Ease of Use } & \multicolumn{2}{c}{ Engagement } \\
\cline { 3 - 6 } & Mean & SD & Mean & SD \\
\hline \multirow{2}{*}{ MT } & Non-Engineering (5) & 4.15 & .74 & 6.07 & .76 \\
& Engineering (7) & 6.12 & .82 & 6.61 & .73 \\
\multirow{2}{*}{ MM } & Non-Engineering (6) & 5.58 & .63 & 6.39 & .88 \\
& Engineering (6) & 5.39 & .39 & 5.92 & .50 \\
\multirow{2}{*}{ SM } & Non-Engineering (8) & 5.63 & .88 & 5.88 & .94 \\
& Engineering (4) & 4.75 & 1.27 & 6.58 & .50 \\
\hline
\end{tabular}

Interviews supported the idea that some non-engineering MT participants found the activity "inconvenient" (P33) or "cumbersome" (P35). Specifically, part of the difficulty that participants had with the MT and MM conditions was the size of the mobile interface. P3 commented “I want to have a bigger size of screen," while P17 commented “...the current one [interface] is a little confusing for a small screen.” One participant with an engineering background (P8) better articulated some of the difficulties with the small screen, stating that "It was bothering to switch between the screen and the scene while programming. It might be cumbersome since people may need to modify -change frequently". Observation of the participants confirmed that it was at times awkward for people to hold the mobile device while triggering the physical sensors, especially for the non-engineering participants. It is possible that engineers, more used to working with technology, had an easier time than non-engineers adapting to the difficulties the tangible interface presented.

There was also evidence that the engineers appreciated the advantages of the MT condition in ways that the non-engineers did not. P1 commented on the benefits of the tangible environment for debugging, stating that "I can test it, it's like a preliminary test 
to see whether sensors are working properly." P26 described the advantages: "I think physically making actions helped me remember -- follow a pattern I've created."

It should be noted that using the menu-based tool also posed its own obstacles for some participants, especially with the need to use the correct sensor IDs and with participants not being sure of which sensor state to select. P36 (MM condition) commented that it was "...not easy to figure out what it is with the pictures in the list; I needed to find sensor ID, and it's a little inconvenient; especially because I was not sure if I should select 'open' or 'close' for a particular item." P12 (MM condition) expressed concern about a potentially lengthy list of sensors when used in real situations that would include significantly more items, which may cause difficulty in finding intended options.

\section{Engagement}

A two-way ANOVA was performed with engagement as the dependent variable and engineering background and condition as factors. There were no significant differences between the effects of condition $(F[2,30]=0.179, p=0.837)$ or engineering background $(F[1,30]=1.023, p=0.320)$ on engagement, and no significant interaction effect $(F[2,30]=2.018, p=0.15$; see Table 1$)$ was observed. Again, the effects of gender were examined using a two-way ANOVA with engagement as the dependent variable and gender and condition as factors. Gender did indeed have a significant effect on engagement $(F[1,30]=5.16, \mathrm{p}=0.03$, Men: $\mathrm{M}=5.98, \mathrm{SD}=0.768$, Women: $\mathrm{M}=6.49$, $\mathrm{SD}=0.708)$. The interaction between gender and condition was not significant $(F[2,30]$ $=0.319, p=0.729)$.

Interview data revealed the specific aspects of the activity that people enjoyed. Results indicate that people liked the immediate feedback they received after programming the 
application, as per the following examples: "Almost instantly you could use an app. Really very fun!" (P17; MT condition), and "I liked how quick it was to program, and how quick the responses were to the actions." (P21; MM condition). Other participants liked particular features of the system, such as: "It's fun when music plays." (P19; SM condition) and "I liked the ability for the system to say something out loud" (P27; MM condition). The most engaging aspects of the system were not unique to a particular condition and engineering background.

Results also demonstrated that participants found possible applications using GaLLaG Strip useful. Most participants gave positive responses to the questionnaire question asking about their future use of this kind of context-aware applications. For example, P17 wrote: "Yes; having a reminder system allows us to stay strongly linked to our goals. Sometimes without external influence, we lose motivation. For the elderly, this would be invaluable!' and P9 stated: “Yes. Reminders to take pills, turn off lights, etc. because I don't always remember." However, there were two negative responses to this question. Although these respondents did not think the feature would be useful to them, they pointed out usefulness to the elderly. P25 wrote: "I guess it might be useful for the elderly (esp. old people living alone or having cognitive impairment) or disabled", P8 wrote: "Not much for me but I could see others (such as elderly) using the system. It would be more useful to learn habits in addition to performing actions. Some things seemed unnecessary but reminders are always good" and P36 stated: “... I thought I might feel suffocated if I really have this system in my daily life. Felt like my privacy \& quietness were being intruded... I thought it will be very useful for elders or patients whose memories are limited, to remind them of important things." 


\section{Ideation Experience}

\section{(1) Effects of physical information}

Gaining ideas. Observation revealed that participants tended to look around the scene while developing their scenario idea in the free-form task ( $59 \%$ of total participants). Even if they were in the $S M$ condition, participants turned their heads to face the scene. For example, P13 from the SM condition stated: "By looking at the room, I was able to get a picture of the whole area... I'm a visually oriented person so [I] like to see the whole picture...looking at the room, I thought of what is in my proximity, and whether it could be better done if I were sitting on the chair." Participants also tended to look around the scene while brainstorming (64\% of total participants, $92 \%$ in MT, $50 \%$ in $\mathrm{MM}$, and $50 \%$ in SM).

Considering intangible aspects. In addition, it was found that the tangible interaction might have interfered with participants' attention toward intangible elements. In the free-form task, only one person in the MT condition used time-date constraints, compared to five in the MM condition, and nine in the SM condition.

\section{(2) Intuitiveness}

Ordering actions. When participants in the MM and SM conditions had to mentally construct a series of actions, the resulting programs tended to be more unnatural. In adding actions for the phrase "leave the living room", one participant (P13, SM condition) added stepping off the mat first and then stepping on the mat, when the order should have been the opposite. Another participant (P16, SM condition) made her application send a SMS message when she closed the door at first. Then, she realized that it made more sense to receive a message before she shut the door, rather than after, 
as the message was a reminder that told her to turn off the air-conditioner before leaving for work. P19 (SM condition) remarked that the most difficult aspect for her in doing the free-form task was to organize her actions (i.e., add actions in the order that she does them in her daily life), stating that "It's hard to organize-- a couple of actions, picking up guitar, Turn on TV, Pick up dumbbells, but hard to order them...what do I do first?" Results indicate that it is likely that the MT condition made the organization of ideas more concrete.

Finding circumstance of an action. Furthermore, it was found that the tangible experience helped people discover particular features of the system. For example, $\mathrm{P}_{3}$ in the MT condition became puzzled because she did not have an idea what sensor to use for the phrase "leave the living room". After wondering for a while, she decided to walk to the entrance door hoping she might discover a clue. By chance, she noticed a sensoraugmented mat that was placed at the edge of the living room area and realized that it was an appropriate item for the phrase.

\subsubsection{Discussion}

The results of the study conducted to investigate benefits of the tangible approach indicate that while the system was usable, people with a non-engineering background perceived it as less easy to use than those with engineering backgrounds. In contrast, the participants with some degree of programming skills considered it easier than participants in the other conditions.

Richness brought by using real-world everyday objects can be considered as augmentations of "role expression" and "hidden dependency" dimensions among the Cognitive Dimensions suggested by Edge and Blackwell (2006). People are familiar with 
the uses of everyday objects and their forms and operations naturally elicit people's recognition and action. Furthermore, as objects are located in a living space, relationships between them are established. For example, the location of objects inside a container depends on where the container is placed, or by sitting on a chair located at a particular spot, a user notices a picture frame in front of the chair. In our case, such richness of artifacts might have been advantageous for the participants with an engineering background because they are more able to handle both the visual interface on a mobile phone and rich information from an environment relatively easily.

Despite the difficulty the non-engineering participants experienced in using the system, benefits of the tangible programming were observed, such as intuitive ordering of actions and diverse ideation with rich physical information. However, the tangible interface tended to distract the participants from the intangible elements of the system. These results indicate that it may be useful to only encourage tangible programming interactions for tasks where the tangible medium is particularly beneficial. The tangible feature might best be used to support people's creation of scenarios that are primarily related to object use and actions. With a GUI, in contrast, people might be better equipped to program applications with non-tangible or global states such as time or weather. Considering advantages of each method, a mixed tangible and menu-based approach seems appropriate to encompass user groups of different programming skills and use cases.

This comparative experiment had some limitations. First, it was conducted in a lab setting, and participants may have displayed more naturalistic responses in a place more familiar to them. Secondly, the sample size was too small to draw solid conclusions about the interaction between condition and engineering major. In addition, the numbers of 
participants of each educational background and gender were not evenly distributed over the conditions. An alternate explanation for the results could be that more women are non-engineers and more men are engineers, so the results obtained might be driven by gender and not engineering background.

\subsubsection{Conclusions}

The results indicate iterative refinement of the interface to allow for more naturalistic physical interactions. Participants found the small screen and switching attention between the scene and the screen problematic in the tangible-mobile tool. It was found that the current interface design should be improved to minimize interruptions of natural user actions. Also, sound could be employed to supplement the visual interface to provide information while the user is manipulating an object, for example, a sound could be played for a newly added action tile so that users do not have to look at the screen to check if it has been added successfully. A better design may also be to have the user record all actions first and then allow them to insert responses between the recorded actions. The shortcomings of the present interface design may have influenced the results of the experiment. Therefore, further study is necessary with a tool improved to better support users' natural performance.

\subsection{Future Work}

The user study of the GaLLaG Strip focused on test of usability and study results indicate that the system allows for easy learning and engaged experience. However, two issues beyond usability from the user study were encountered: (1) How to expand the current programming functionality so that it can allow diverse implementations but is still easy to use, and (2) how to foster user exploration of rich and meaningful solutions. 


\section{Balancing Between Simplicity and Expressiveness}

As an initial prototype of a programming interface, GaLLaG Strip was designed to provide fundamental programming elements. The interface provided a simple and thus easy to learn user interface, and users can still create applications diverse to some degree. However, participants of the user study found it necessary to expand the functionality such as logic elements (e.g., “OR”, “IF...ELSE”), recognition of current states (e.g., to enable development of this type of idea "trigger a sound if he did not use the dumbbell after 6 AM"), calculation of time duration (e.g., to enable realization of this type of idea "tells him to do work out if he watches TV longer than an hour"), etc.

However, inclusion of further functions may need to be implemented carefully as doing so can bring complexity and impair learnability. Importantly, expressiveness, the ability to produce a wide range of application types, and ease of use and learning are primary concerns in developing end-user programming tools (García-herranz et al., 2010). It is considered important to balance simplicity with expressiveness in ways that prioritize end-user capacity to create a wide range of experiences. In developing a tool for nonexpert users, simplicity is weighed over functionality. Resnick et al. (2005) emphasized the importance of simplicity as one of the key design principles for creativity support tools, asserting that reducing the number of features can improve the user experience (in the context of their observation of development of a programmable LEGO Brick in the mid-1990s). The current expressiveness of GaLLaG Strip should be further expanded to some degree, including features frequently wanted by users. Given that there are no decisive rules on how simple tools should be or how much tools can sacrifice in their expressiveness, iterative evidence-based study is necessary to reach appropriate balance between a tool's expressiveness and simplicity. 


\section{Supporting Creativity}

In the user study conducted with GaLLaG Strip, participants differed with respect to creativity in generating application ideas and satisfaction toward the ideas developed. While some participants produced rich ideas reflecting various daily life situations and involving many artifacts, other participants showed a lack of such quality. For example, during the task of programming personal scenarios, P3o generated the following idea:

Turn on TV > Say "Do exercise" > Take the dumbbell > Play "Achievement sound" > Play "Cheerful music"

This idea is simply a slight extension of the scenario provided for the first task. In contrast, P14 produced the following idea:

Arrive at home $>$ Say "Welcome home. Time to take shower" $>$ Enter the shower stall $^{* *}>$ Continued for 10 minutes*, Say "No more" $>$ Come out of the shower stall $>$ Play "Reminder sound" $>$ Say "Time for some food" $>$ Open the refrigerator** $>$ Say "Have some fruit" > Close the refrigerator $>$ Say "Bon apettit"

* As GaLLaG Strip did not support this time counting, the participant was asked to omit it when programming.

** As the lab did not provide those objects, P14 was asked to use other objects in the lab for programming and testing.

Compared with P30's idea, P14's fluency in recalling his daily life demonstrated abundant awareness about what he desires or should do. Participants often expressed their discomfort with the sophistication of their application ideas. For example, P3o stated that “Though I feel I can make it better, I don't know what I can do more." P16 said that "I know there are many more things that can be done, but I only made it like this." In addition, P24 stated that "To me, it was hard to come up with scenarios. System offers many options such as play music, etc. but cannot help but think of reminders. Especially it was hard to think of more than two steps..." These observations indicate that some users may need support when designing, especially during the start-up phase. 
A number of existing studies investigating end-user development have emphasized support as an important factor. The scarcity of ideas to attempt or uncertainty about requirements in meeting their goals is considered as one of the primary barriers that users encounter (Cao, Fleming, \& Burnett, 2011; Ko, Myers, \& Aung, 2004). With the concept of "domain-oriented design environments", Fischer asserted the necessity to support people's creativity going beyond simple construction kits to "assist their truly interesting objects" (Fischer, 1993, 2007). To the best of my knowledge, there is no research that recognizes the need to support a user's ideation with toolkits for end-users creation of context-aware applications. One reason for this gap in the research may be that existing ubiquitous computing approaches tend to address the most apparent problems and then apply a range of relatively straightforward solutions. However, in moving beyond today's smart home applications to the realm of behavior change, socalled "wicked" problems are encountered. That is, the problem of behavior change shares much of characteristics of wicked problems proposed by Rittel \& Webber such as "There is no definitive formulation of a wicked problem", "Solutions to wicked problems are not true-or-false, but good or bad", and highly dependent on the perspective of the stakeholders involved (1973), which require stakeholders to exert more resourceful, collaborative, and nuanced approaches to problems.

\subsection{Summary and Next Steps}

In this chapter, GaLLaG, a suite of hardware and software for building sensor-based feedback systems for just-in-time interventions was presented. It is characterized by several features: rule-based event-driven composition, user action detection with simple two-state sensors, and multiple feedback channels including SMS, sound and music playback, and appliances. Then, GaLLaG Strip, a visual programming interface for 
GaLLaG was described, which was developed to allow users with little or no programming experience to program GaLLaG applications. The user study of GaLLaG Strip indicated that participants, including participants with no programming skills, were able to complete the tasks after viewing the relatively short video instruction. In addition, participants were highly engaged in the experience. These findings support learnability and adoptability of end-user programming tools to create context-aware applications for behavior change. However, a need for further work to expand the current programming functionality that allows more complex composition but remains simple enough for users to easily learn and use was evident. Another issue highlighted was supporting users' creativity in the generation of application ideas. Those topics are not addressed further in this dissertation, but considered important for improved usefulness and user experience.

In chapter 4, development of the tutorial for users' behavioral plan creation is described. First, the fundamentals that directed development of a design support tool are detailed. Second, an initial prototype of a design support tool and user study conducted to investigate its effectiveness is presented. Next, a revised version of a tool and user study is described. 


\section{SUPPORT FOR BEHAVIORAL PLAN CREATION}

\subsection{Approaches and Initial Prototype}

Based on the rational problem-solving paradigm and behavior change techniques reviewed in chapter 2 , fundamental approaches in designing a behavioral plan creation support were established. Provision of a structured process consisting of several steps is pursued, and initial steps of the process should lead users to reflect on an issue of interest and determine (multiple) sub-parts clearly. In addressing each part, the process invites users to learn and apply general strategies to obtain appropriate ideas easily. Three components of a behavioral plan for an issue were identified: (1) goals, attainment of which may eliminate or relieve the issue; (2) action plans to facilitate attainment of the goals; and (3) self-tracking methods to assess how successfully the goals were attained.

Following the framework above, a low-fidelity prototype (i.e., PowerPoint presentation) of the tutorial was created, which was fully scripted and images were provided in succession. The tutorial consisted of five steps: (1) choosing a (target) behavior to attempt to change, (2) setting a goal, (3) generating action plan ideas by applying behavior-change techniques, (4) formulating a final plan, and (5) devising self-tracking measures to determine if the goal was accomplished. The tutorial included visual and verbal materials that described tasks to complete and concepts of behavior change techniques. Because it assumes presence of a facilitator, the tutorial also defines their remarks and actions. In the following section, details of the prototype design are detailed by presenting how each of the three components is achieved. 


\section{Setting Goals}

It was possible that users might have broad ideas about their problems but are not aware of what specifically they should do. Thus, several steps were implemented to lead users to identify sub-parts of a given issue and reach tangible goals. The tutorial first asks users to externalize aspirations in their minds and then choose one that they would most like to pursue. For easy understanding on what they are supposed to think of, a prompt to think about recent New Year's resolutions is given (Figure 15, first row). Then, in an attempt to promote coverage, a list of domains to consider (health, self-development, work, etc.) is presented (Figure 15, second row). Once users generate all ideas, they are asked to select one to work towards. Users then learn about the concept of sub-goals and identify sub-goals of the goal previously selected (Figure 15, third row). Finally, users are asked to choose one or more goals among the generated goals.

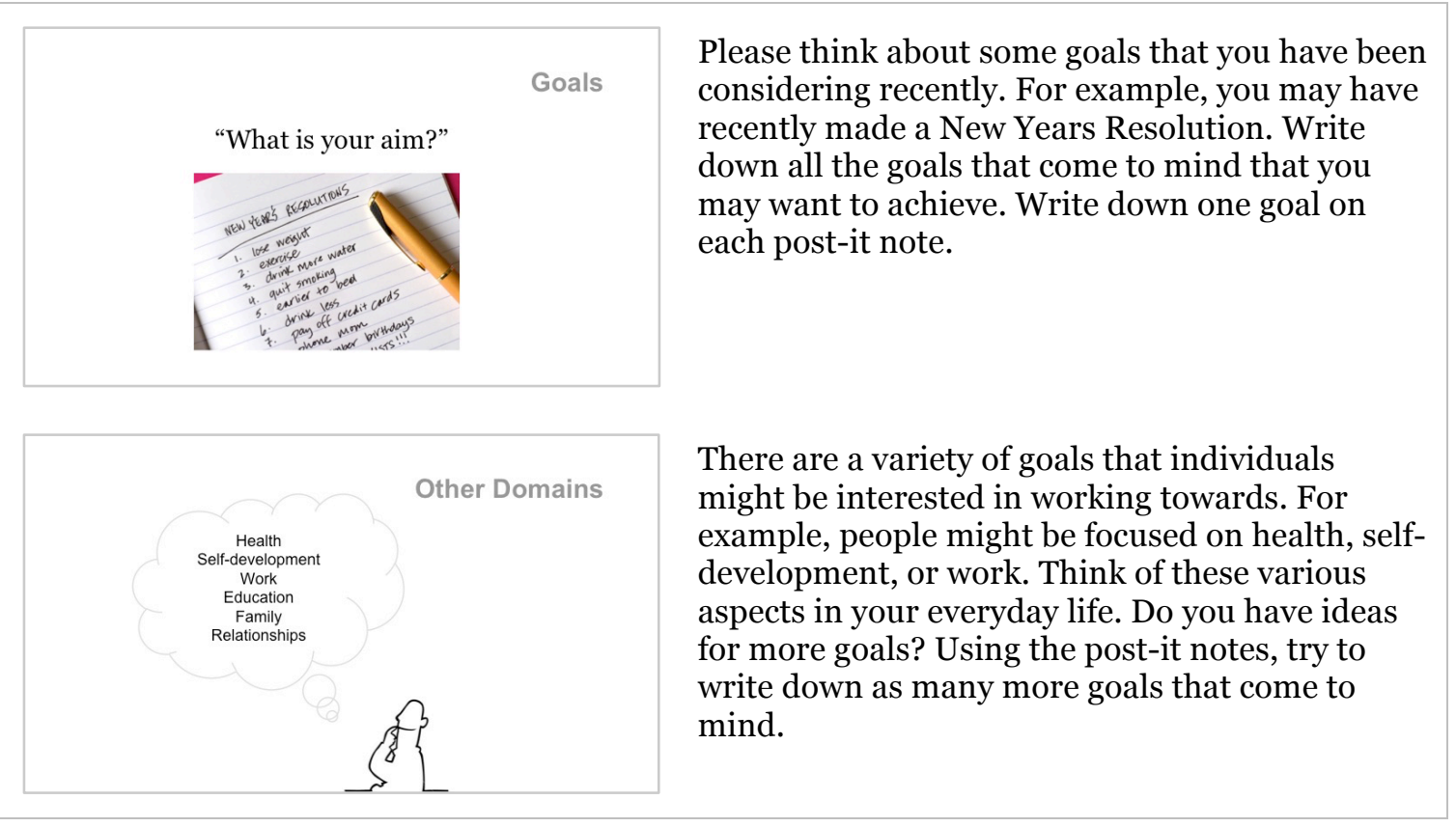




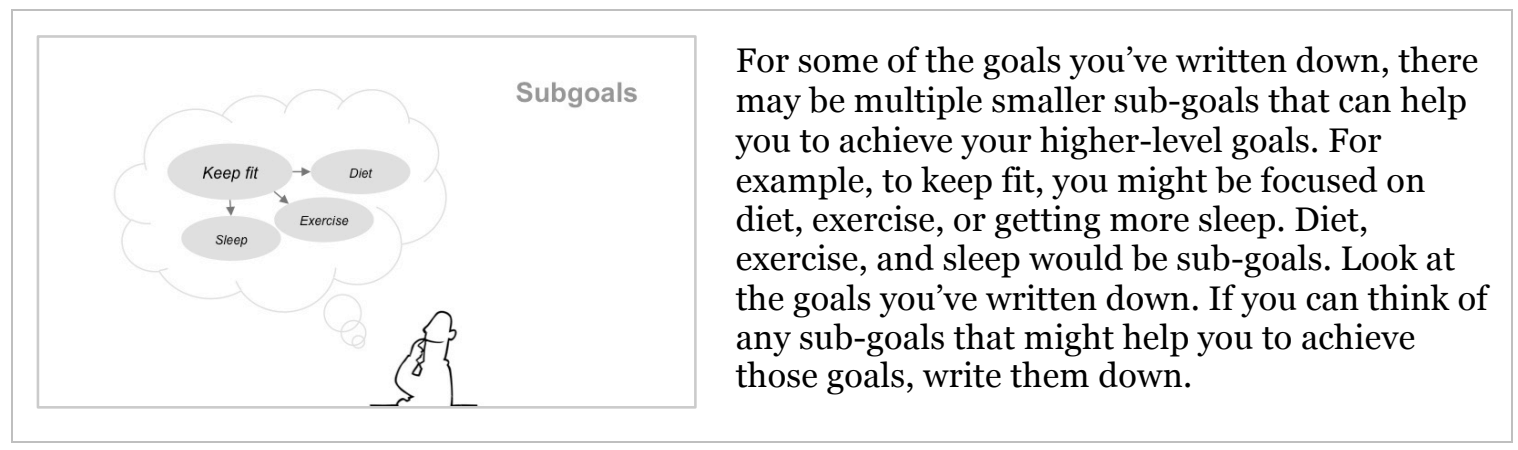

Figure 15. Initial Prototype of the Tutorial for Supporting Behavioral Plan Creation: Setting a Goal

\section{Making Action Plans}

After setting a goal, users devise a plan for goal attainment through three steps involving different approaches for ideation. First, users are asked to generate behavioral plan ideas with a prompt shown in Figure 16 (first row). Then, they are asked to talk about their past experience regarding the chosen goal, which is followed by generating further ideas or rewriting their current plan ideas based on their reflection (Figure 16, second row).

\begin{tabular}{|c|c|}
\hline $\begin{array}{l}\text { Plan Ideas } \\
\text { "How will you reach your goal?" }\end{array}$ & $\begin{array}{l}\text { Now it's time to think of some plans to help you } \\
\text { reach your goal. It is known that having } \\
\text { appropriate plans is a key factor for goal } \\
\text { achievement. }\end{array}$ \\
\hline $\begin{array}{l}\text { Specify what you will do, when you will do it, and how } \\
\text { Incorporate means to ensure your success }\end{array}$ & $\begin{array}{l}\text { Formulate plans to help you reach your goal. } \\
\text { These should be effective and achievable, } \\
\text { specifying what to do, when and how to do it, } \\
\text { and outlining ways to ensure its completion. It's } \\
\text { good to think of multiple number of plan ideas. } \\
\text { You can choose a better one later. }\end{array}$ \\
\hline & $\begin{array}{l}\text { One tip for easy creation is to single out all small } \\
\text { or big ideas first and then sort, combine, or } \\
\text { shape them into a plan. }\end{array}$ \\
\hline
\end{tabular}


Past Experience

Look back on any past experiences regarding this goal.
By reflection on your past experience regarding the goal, you may be able to find some lessons for making plans.

OK, first, can you tell me what you did regarding this goal, and what it resulted in?

With such experience, do any thoughts or ideas regarding plans come to your mind? That is, you may want to make changes to the present plan or come up with new plans with them. If so, please work on them, writing down ideas that come to your mind.

Figure 16. Initial Prototype of the Tutorial for Supporting Behavioral Plan Creation: Making Action Plans

After the ideation with the past experience, a set of 13 behavior change techniques that covers most categories identified in the behavior change techniques taxonomy (Michie, et al., 2013) are provided, participants are asked to choose three techniques to incorporate into their further ideation (i.e., generate ideas on how to apply a technique to their pursuit of goals). Users are told the following: "Here are behavior change techniques suggested by the experts to use, for successful goal achieving. Please review these and understand what each technique is. After you are familiar with the techniques, choose three, which you will use to revise the current plan(s) or think of new ideas." A quantity of three was chosen to prevent participants from being overwhelmed with too many techniques to work on and allow some room in case users preferred multiple techniques. Users then look through description of each technique, which consists of a label, one-sentence definition, and use example presented in comic strip style (Figure 17). Once users finish ideation with the behavior change techniques, they are asked to look through generated ideas and finalize the plan. 


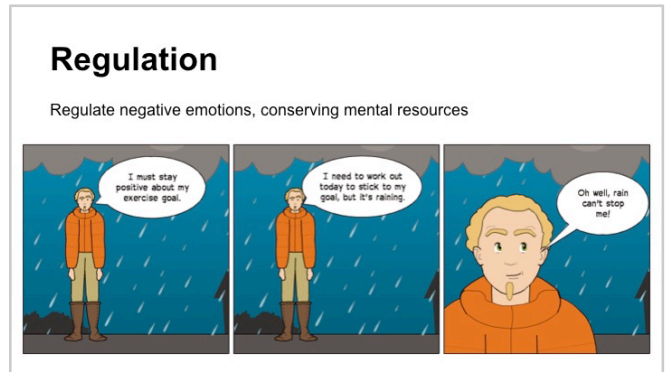

\section{Facilitating}

Change surroundings to encourage activities

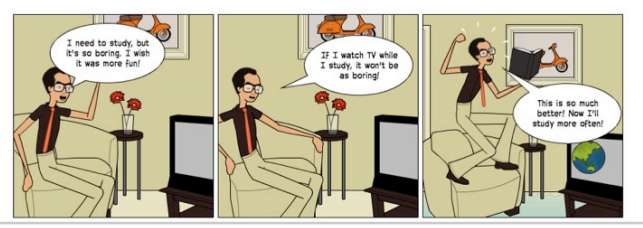

Figure 17. Initial Prototype of the Tutorial for Supporting Behavioral Plan Creation: Exemplar Slides of Behavior Change Techniques

\section{Establishing Self-tracking Methods}

After users finalize their behavioral plan, they are introduced to the concept of selftracking (Figure 18, first row), and are provided with two options for the method: structured and unstructured (Figure 18, second and third rows). Of the two self-tracking methods, users are asked to choose one they think they would most likely be able to implement easily and that would be most helpful for them in fulfilling their goals and plans. Users who chose the structured journaling develop questions to answer, and both options require users to set specific times of day to complete their journaling.

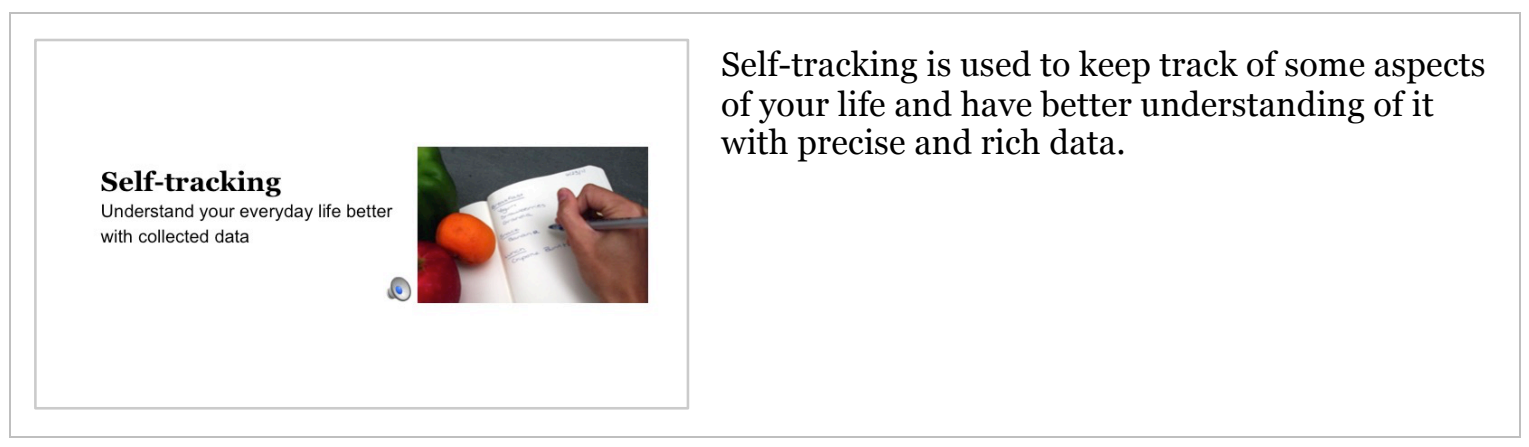


Structured Questions

Fill out same questions at the end of each day.

I met my goal of running 30 minutes today.

Response Options: yes / no

I put my 30 minute run into my schedule.

Response Options: yes /no

- If you did not meet your goal, what made it hard?

Response Options:

Overall, how satisfied or content did you feel today?

Response Options: 0-not at all - 10 - completely

Unstructured Journaling

Pick up to three times each day to journal about your goal and plan. Write whatever you want but consider answering these sorts of questions.

Did I reach my goal today?

If so, what helped me to reach my goal?

- If not, what kept me from reaching my goal?

-Is my plan helping?

Can limprove my plan in some way?
First, "Structured Questions Approach". In this style of tracking, the purpose is to gather very specific information that you think will help you understand how you are doing with enacting your plans to reach your goals. It involves creating very specific questions that you think will help you to understand not only if you met your goal or not but factors that might impact your goal and plan.

For example, lets say that you had a goal of running thirty minutes every day. Further, as you think about it, you think your mood, your confidence level in running, and running with others will greatly impact if you will be able to run or not. Based on this, you come up with the questions on the slide.

To use this style, you will need to come up with a set of concrete questions that you can ask yourself every day, and set a time in the morning, afternoon, or evening to answer.

This approach is used if you aren't quite sure what you should be tracking or if you just don't like all of the structure of the other technique. For this one, the goal is to set aside times throughout the day to write down any thoughts or observations you might have related to your ability to reach your goal. During these times when you write, spend your time writing any observations you may have made about your goal and/or plan. You might try to answer questions like, "Did I reach my goal today?", "If so, what helped me to reach my goal?", "If not, what kept me from reaching my goal?", "Is my plan helping?”, "Can I improve my plan in some way?"

We recommend that you do this, at the very least, one time at the end of each day but you could also set a time in the morning, afternoon, and evening to take 5 minutes to write down any thoughts you have on your goal.

Figure 18. Initial Prototype of the Tutorial for Supporting Behavioral Plan Creation: SelfTracking Methods

\section{Revising}

The tutorial assumes users' iterative work to obtain behavioral plans optimized to their needs. That is, after users implement the goal, plan, or self-tracking for a certain period 
(e.g., two weeks), they revise the current plan. Users first check goals and decide whether to switch to others, add another goal, or modify details on the current goal description. For the action plan, users are asked to revise their current plan based on their implementation experience. If they want, they can attempt some other behavior change techniques, that is, select one from the list, learn about it conceptually, generate ideas on how to apply it to their goal pursuit, and combine those ideas into the current plan. Users also determine if the current self-tracking method is appropriate.

\subsection{User Study of the Initial Prototype}

To evaluate usability and usefulness of the prototype, a user study in which participants created or revised behavior change plans to their self-identified problems with the prototype was conducted. Specifically, the study investigated if there were difficulties in participants' ability to follow the prototype and whether behavior change plans were created or revised as the prototype intended.

\subsubsection{Method}

\section{Procedure}

During the study, participants completed three one-on-one sessions over a two-week period. In session one, participants developed their initial plan, and in session two (after implementing the initial plan for one week), they revised the plan. In session three, participants again revised their present plan after one-week implementation. Although the study ended after session three, participants were asked to revise the plan supposing they continued to use it. Session two and three were scheduled at the conclusion of the prior meeting (within six to nine days). For the self-tracking, participants were given a pocket-sized notepad in which they wrote down their behavioral plan to work on and 
times to journal until the next session. Participants who chose the structured journaling method wrote down questions on the notepad.

All sessions were held in the same room, which was a small workshop-like room on Arizona State University's Tempe campus. By design, the sessions were delivered by individuals with no formal training in providing behavioral counseling to ensure clinical training did not contribute to any potential effects of the session design.

\section{Participants}

Using mailing lists, recruitment emails were distributed to graduate and college students at Arizona State University. Age constraint was set to recruit individuals 18 years old or older. There were no further exclusion and inclusion criteria. As compensation for participation, each participant was given Amazon gift cards over the three sessions (\$20, $\$ 30$, and $\$ 40$, for a total $\$ 90)$.

Participants were a convenience sample ( $\mathrm{N}=11 ; 9$ female and 2 male) of college and graduate students at Arizona State University. Ages ranged from 18 to 39 years of age. The types of goals generated by the participants spread over various domains including health, study, career, finance, spirituality, etc.

\section{Measures and Analysis}

To examine perceived usability, a survey composed of 7-point Likert scale questions was developed, and participants completed it at the end of each session. Specifically, the survey aimed to investigate: (1) ease in learning concepts and completing tasks, (2) engagement, and (3) satisfaction with final plan created. To assess usefulness of the prototype, the quality of participants-created behavior plans and performance during the 
sessions were examined by examining participant-created plan descriptions (i.e., text written on a notepad by a participant finishing each session) and session logs recorded by two researchers who administered the sessions. The two researchers read those materials repeatedly and salient issues found by each researcher were compared to locate those that both commonly noticed.

\subsubsection{Results}

\section{Perceived Usability}

Table 2 lists averaged points of participants' responses to questions related to the degree of ease in understanding concepts such as instructions of tasks and behavior change techniques and working on tasks. For questions of positive statements, participants chose between 5.7 and 6.9 in average, and for questions of negative statements, between 1.3 and 2.0. Overall, results indicate that participants did not have any significant difficulties.

Table 2. User Study of the Initial Prototype of a Self-experimentation for Behavior Change Tutorial: Perceived Easiness

\begin{tabular}{lccc}
\hline \multicolumn{1}{c}{ Questions } & Session 1 & Session 2 & Session 3 \\
\hline I think the session was difficult to follow. & 1.4 & 1.3 & 1.5 \\
I think the session went smoothly. & 6.8 & 6.7 & 6.9 \\
$\begin{array}{l}\text { I think the materials and instructions were clear and } \\
\text { easy to understand. }\end{array}$ & 6.7 & 6.9 & 6.8 \\
$\begin{array}{l}\text { It was difficult to focus while thinking of plans. } \\
\text { I was able to easily understand the concepts of the }\end{array}$ & 2.0 & 2.0 & 1.6 \\
$\begin{array}{l}\text { Behavior Change techniques. } \\
\text { I felt overwhelmed when presented with the Behavior } \\
\text { Change techniques. }\end{array}$ & 6.5 & 6.7 & 6.6 \\
$\begin{array}{l}\text { I was easily able to determine what Behavior Change } \\
\text { techniques would and would not work for me. }\end{array}$ & 5.7 & 6.6 & 1.7 \\
\hline
\end{tabular}


For questions designed to investigate if participants felt engaged during the sessions, participant responses are displayed in Table 3. Considering points between 6.0 and 6.6 of the positive statement questions, and between 1.4 and 2.0 of the negative statement questions, results indicate that participants had a fun and satisfying experience.

Table 3. User Study of the Initial Prototype of a Self-experimentation for Behavior Change Tutorial: Perceived Engagement

\begin{tabular}{lccc}
\hline \multicolumn{1}{c}{ Question } & Session 1 & Session 2 & Session 3 \\
\hline The tasks did not catch my interest. & 2.0 & 1.4 & 2.0 \\
My experience with the session was rewarding. & 6.6 & 6.5 & 6.3 \\
$\begin{array}{l}\text { I felt the experience of creating behavior change } \\
\text { solutions was fun. }\end{array}$ & 6.5 & 5.9 & 6.0 \\
\hline
\end{tabular}

Results provided in Table 4 reveal that participants were satisfied with self-created plans overall.

Table 4. User Study of the Initial Prototype of a Self-experimentation for Behavior Change Tutorial: Satisfaction with Self-created Plans

\begin{tabular}{lccc}
\hline \multicolumn{1}{c}{ Question } & Session 1 & Session 2 & Session 3 \\
\hline I am not satisfied with my final plan. & 1.8 & 1.2 & 1.4 \\
I am excited to carry out my plan. & 6.6 & 6.5 & 6.5 \\
I think my plan fits my lifestyle well. & 6.3 & 6.2 & 6.0 \\
\hline
\end{tabular}

\section{Plan Creation}

Three issues were found during examination of participants-generated plans and participants' performance during the sessions.

(1) Plans broadly defined

First, participant-generated plans were not specific enough. In session one, the prompt to think about sub-goals appeared to be valuable for leading participants to transform 
items that were generated in the first step into more specific forms. However, final goals set were still broad. Plans for the goals were also defined broadly. The outcomes by P4 and P6 below exemplify such problematic qualities. P4's goal, “eat healthier”, and parts of her action plan such as "allow more time of cooking food" and "Build a better time schedule to accommodate making new food to eat and enjoy" lacked details on how they would be carried out. As another example, "Wholesome food diet", P6's plan item defined in sessions two and three, included no details on how to realize the goal.

Table 5. Exemplar Participants-generated Plans of the Initial Prototype User Study

$\mathrm{P} 4$

Session 1

Goal: Eat healthier

Plan: Shop for healthy groceries to be incorporated into recipes I will be able to make. If the temptation of junk food arises, I will counter the urge with a packed lunch from home and stick to that food only. If going out to eat is inescapable, it is ok to get something nutritious from the menu!
Session 2

Goal: Eat healthier

Plan: I will make sure to bring a list with enough food with me for when I go grocery shopping to last me a week (at least). I will remember how good the food I made was and think about how good new recipes will be to try. This will (hopefully) make me feel better still. I will take this food with me for school/friend time to cope with the temptation to buy extra food that is unhealthy.

P6

Session 1

Goal: Get physically fit

Plans: Running every morning.

No crash diet.

Sleep for 7 hours from 12 to 7 AM.

Drink lot of water.

Remind yourself or make my friends remind me of my goals.

Reward myself (if you follow
Session 2

Goal: Get physically fit

Plans: Running every morning.

Wholesome foods diet.

Sleep can be fragmented but try to cover 7 hours.

Drink lot of water.

Regulation technique -Regulate procrastination. Coping planning -- Think ahead of time \& account for
Session 3

Goal: Eat healthier

Plan: Allow more time for cooking food

Try for 4 new different recipes per week

Build a better time schedule to accommodate making new food to eat/enjoy. 


\begin{tabular}{lll}
\hline it throughout for 2 weeks). & potential obstacles. & potential obstacles. \\
& Reinforcement -- Use & Reinforcement -- Use \\
immediate rewards (like & immediate rewards (like \\
playing for 1 hour if work & playing for 1 hour if work \\
done). & done). \\
& Find an active partner who is \\
& doing similar tasks (for \\
& keeping me motivated). \\
\hline
\end{tabular}

(2) Less engagement with behavior change techniques

Second, participants were less engaged with behavior change techniques. Frequently, behavior change techniques served as a way for participants to attribute labels to elements they had devised in the previous steps. As illustrated in Figure 19, participants chose "Goal-setting" and "Monitoring" relatively frequently in session two, which were already realized as part of the process. Although some participants tried to generate further ideas with techniques chosen, they often failed in defining their actions specifically. For example, P6 chose a technique, "Coping planning”, and his plan with this technique "Think ahead of time \& account for potential obstacles" is simply a restatement of the concept of the technique.

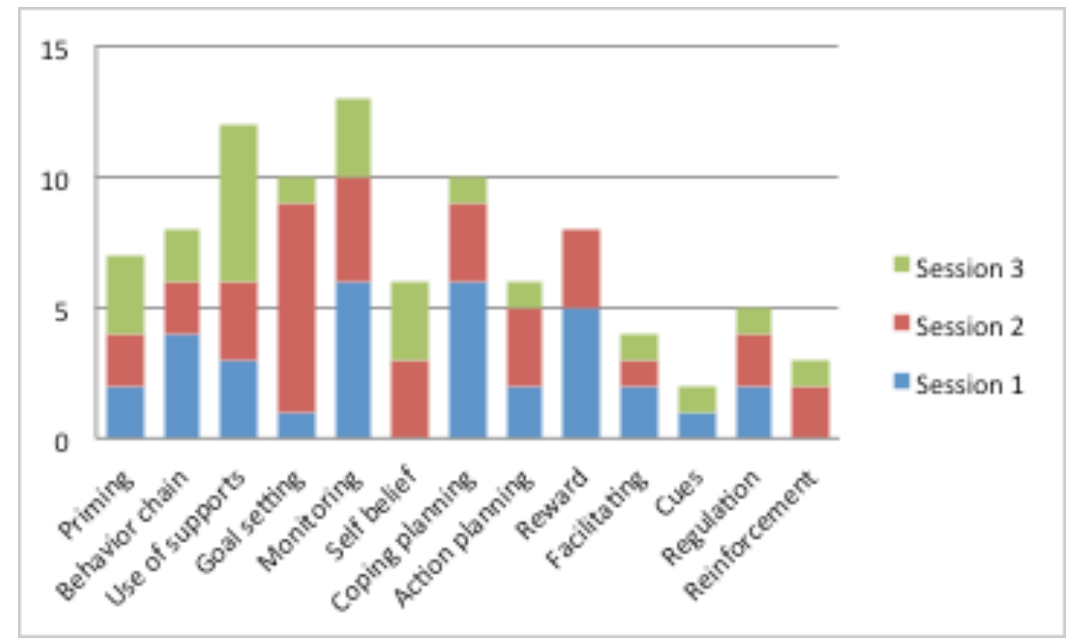

Figure 19. Frequency of Behavior Change Techniques Chosen 
(3) Less attention to environmental factors

Finally, as illustrated in Figure 19, frequency of choice varied between participants. In particular, "Cues”, "Facilitating”, and “Reinforcement”, which involve augmenting environmental and external factors for promoting automatic behavior, were chosen less frequently compared with other options such as "Coping planning”, "Monitoring”, and "Reward". Assuming the necessity of balancing between automatic and rational behavior in promoting behavior change (Hekler, Burleson, \& J. Lee, 2013), such bias is considered problematic.

\subsubsection{Discussion}

Results obtained from the user study indicate that participants did not have significant difficulty during the sessions and had a fun and satisfying experience. However, participants-produced plans were quite broad and behavior change techniques were not explored deeply. The step-by-step structured process approach appears adequate, and participants were easily able to understand the goal-plan construct and self-tracking with the given description. However, the current method to induce creation of specific plans is not considered sufficient. Although participants were asked to produce "sub-goals" and notified on "specificity" (specifying what to do, when, and how to do it, and outlining ways to ensure its completion, see the first row in Figure 16), they did not elaborate as instructed, as was expected. The tutorial also failed to facilitate participants' active use of behavior change techniques. In addition, their reduced attention to manipulating environmental factors is considered problematic. Although there is no clear evidence on why behavior change techniques on environmental aspects were chosen less, one assumption could be people's typical tendency of attributing their failure in behavior change to their lack of motivation or will power. 
4.2.4 Conclusion

Overall, the fundamental structure of the tutorial is reasonable from a user perspective and effective in establishing behavioral plans, and the various integrated concepts are understandable for users via appropriate delivery methods. However, the tutorial should be revised to help users become more clearly aware of attributes desired in defining plans and immersed in working with behavior change techniques.

\subsection{Revised Prototype}

Based on the results of the user study, the initial prototype was revised, mainly by integrating two features. First, in response to the issue of participants' under-specifying goals found in the user study, the concept of SMART goals (Latham, 2003) was included during the goal-setting phase. The SMART (Specific, Measurable, Actionable, Realistic, and Timely) goal concept is a reinterpretation of Locke and Latham's goal setting theory (Locke \& Latham, 2002). According to the SMART concept, goals that meet each word in the acronym (e.g., specific, measurable, actionable) will likely be more useful for supporting behavior change. The revised prototype teaches this concept to users and invites them to make SMART goals as their final outcomes of the goal-setting steps. Users generate SMART goals by going through the following steps: (1) reflecting on any current issues that they want to work on, (2) learning about the concept of "behavioral goals" and contrast those with "outcome goals" to ensure the goals were behavioral targets, and (3) learning about the concept of a SMART goal with instructions on how to create one.

Second, the revised prototype provides an organizing structure to help individuals in the selection and personalization of behavior change techniques. Behavior change 
techniques are categorized via a meta-model of behavior. The meta-model was created based on two existing meta-models: Fogg's behavior model (Fogg, 2009) and Michie et al's COM-B model (Michie, van Stralen, \& West, 2011), which were initially developed to help professionals create interventions. Specifically, behavior change techniques are grouped into four domains: opportunity (availability to engage in a behavior), triggers (prompts to perform the behavior), ability (having the required skills or attributes to perform the behavior), and motivation (drive to do the behavior). Fogg's model proposes "Motivation, Ability, and Triggers", while Michie et al's COM-B model emphasized "Capability, Opportunity, and Motivation". Ability and capability are collapsed in the present research because they are similar constructs. In contrast, triggers and opportunity are considered to be related but distinct. The following is a list of techniques categorized with the model:

- Trigger: Define a trigger, Information or inspiration as triggers, Counteracting negative emotional triggers

- Opportunity: Find the opportune or dangerous time and place, Turn off your "auto-pilot", Make it the "default" option

- Ability: Script critical actions, Shrink the change, Build habit chains

- Motivation: Define your inspiration, Ride the wave, Reward yourself.

With those two features, the initial prototype was revised as described in the following section.

\section{Setting Goals}

Figure 20 displays slides and verbal instructions provided by a facilitator for the goalsetting option. While the initial prototype asked participants to think of "goals" at first, 
the revised prototype uses the word "issues". By using "issues", some unsatisfying aspects might first arise in the user's mind before they conceive what should be achieved.

After selecting the issues, users choose one to work toward. Then, they learn about the concept of "behavioral goals" instead of "sub-goals" of the initial prototype. This approach was considerd as better for helping users genenrate more specific and actionable goals, which are closer to the concept of SMART goals and thus users can easily obtain them. In the next step, users learn about the concept of SMART goals and adapt behavioral goals produced previously to the newly learned concept.

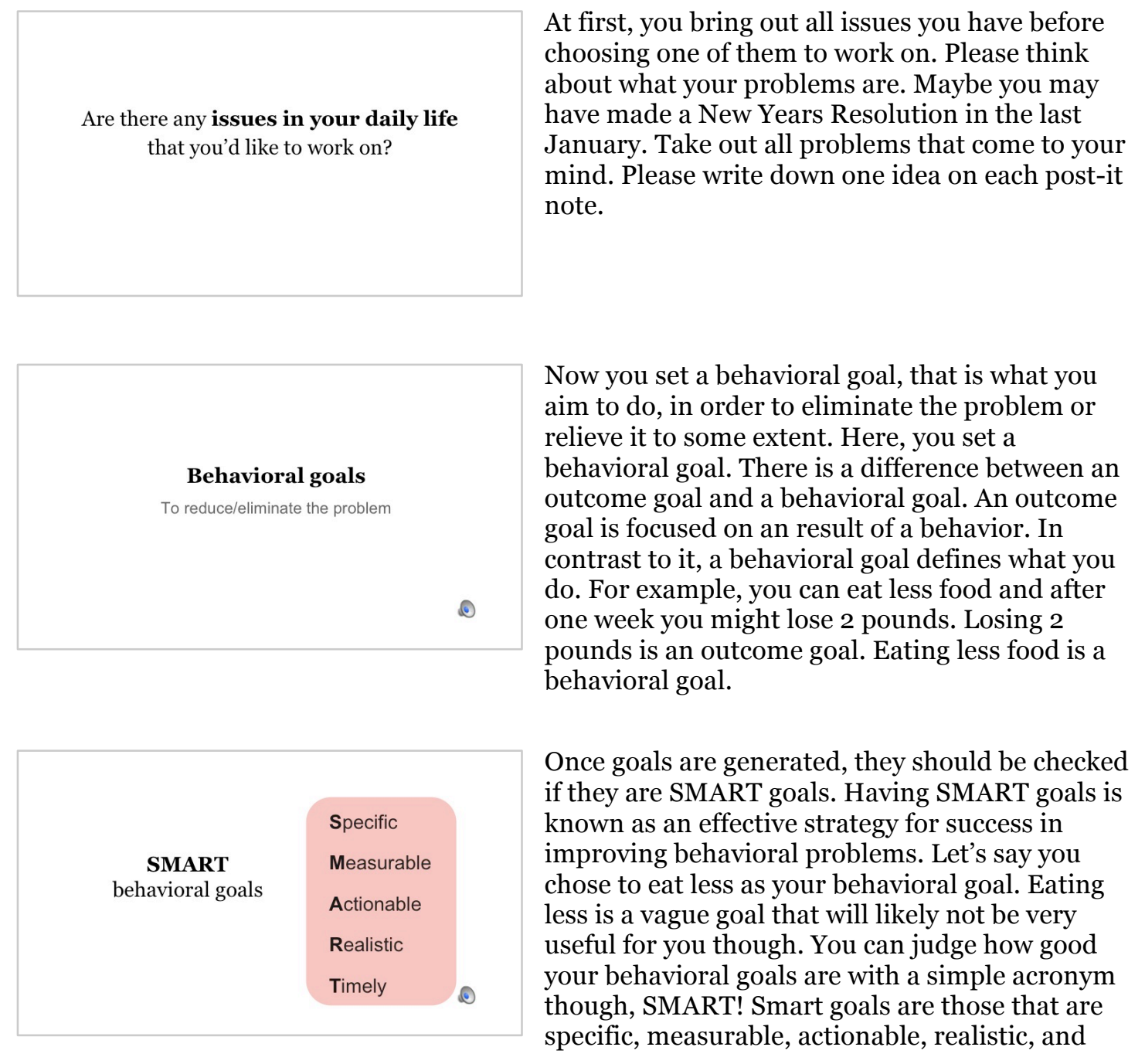


timely.

So, if your behavioral goal is to eat less, you could make it specific by choosing a calorie goal such as 1500 calories per day.

Measuring calories though is very difficult; it is possible but a pain and most people don't continue to measure it for a long time. To make your "eat less" goal more measurable, another option you could try would be to pick specific foods to set goals around. For example, you could set a goal related to drinking soda and other sugar-sweetened beverages. It is easy for you to measure how much soda you drink in a day (e.g., cans, glasses, or bottles of soda) so you could set yourself on a goal of drinking no soda.

Drinking no soda or other sugar sweetened beverages is now measurable and it also happens to be actionable as you know exactly what you need to do (or in this case, not do).

Let's say you currently drink 4 cans of soda a day though...dropping to no sodas might not be very realistic for you. To make your behavioral goal more Realistic, you could try for a more manageable goal such as to set a limit of no more than 2 sodas per day.

Finally, since this goal is being set within a specific time, within a day, you can also know when and how often to do it (i.e., no more than 2 sodas per day). Timely also is there to remind you that the goal might change over time. For example, your goal of no more than 2 cans a day of soda might be realistic right now but later, once 2 cans is a realistic behavioral goal for you, perhaps you can further cut down to 1 or even no soda per day. The "Timely" part of a SMART goal is there to help you remember to adjust your goals based on your progress.

Figure 20. Revised Prototype of the Behavioral Plan Creation Support: Setting Goals

\section{Making Action Plans}

In creating initial plans, users learn about four behavior-change techniques (trigger, opportunity, ability, and motivation) and generate ideas on how to apply them to the goal pursuit. Finally, users shape their final action plan by organizing the ideas 
generated. This revised version removed the two steps (Figure 16) that came before the behavior change techniques step in the initial prototype. The aim was to strengthen participants' engagement with behavior change techniques. As illustrated in Figure 21, behavior change techniques are presented in a different style. Previously, short textual description and an example depicted in a comic-book style were provided (Figure 17). After the changes, the revised prototype offers much longer verbal description that explains a technique concept using additional details and multiple examples.

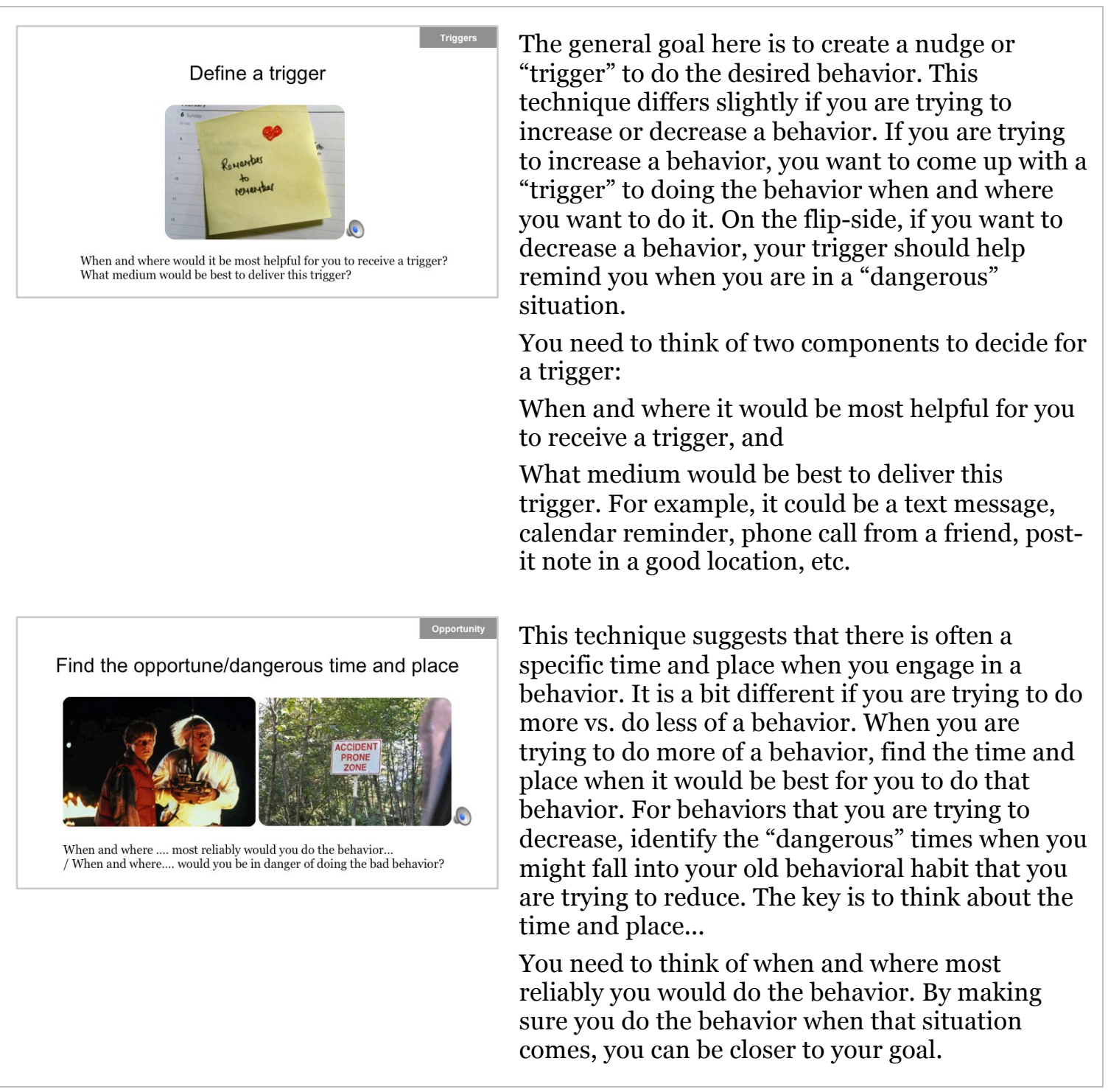


/ when and where you would be in danger of doing the bad behavior. By making sure you avoid the behavior at that situations, comes, you can be closer to your goal.

Figure 21. Revised Slides of Behavior Change Techniques

In revising plans, users are presented with the meta-model phrase and each technique they previously tried is linked to the meta-model (Figure 22).

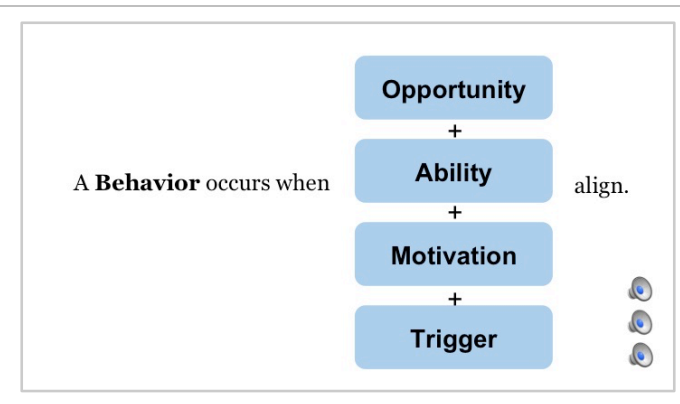

At the last meeting, you were given these four behavior change techniques; "Find the opportune/dangerous time and place", "Script critical actions", "Define your inspiration," and "Define a trigger".

Before we tell you about a few more techniques, we want to give you a model to think about for understanding how behavior works. In brief, a behavior only can occur when the situation to do it, the ability to do it, the motivation to do it, and the trigger telling to do it are in alignment.

For example, let's say your behavioral goal is to go on a 30 minute walk every day. To accomplish a 30 minute walk you need the opportunity to walk, such as finding a good walking route. You need to have the ability, such as having enough time to take out of your day to walk 30 minutes, the right shoes so that you don't hurt yourself, or to be fit enough to walk 30 minutes without causing harm. You also need to have some degree of motivation to walk 30 minutes. Finally, there should be some trigger or reminder that tells you to start it now. As a reminder, we taught one technique from each of these domains.

Each of the behavior change techniques given last week is related to each of them. The technique, "Find the opportune/dangerous time and place", is to increase opportunity that you start a desired behavior or avoid an undesired habit. By specifying situations and being aware of them, opportunity that you start a desired behavior can be increased or opportunity that you avoid an undesired habit can be decreased.With pre-defined action steps, you can manage an important situation promptly. Your inspiration boosts your motivation. And you were invited to build a trigger to nudge yourself.

Figure 22. Slide for Self-diagnosis with the Meta-model

Participants are asked to self-diagnose whether there may be a lack of opportunity, ability, motivation, or triggers when it comes to enacting their goals, which is prompted 
by the following command: "The next step is to continue to refine your plan by working on better picking up your techniques. When it comes to picking the right behavior change technique, a good strategy is to figure out if you aren't achieving your behavioral goal because of an issue with opportunity, ability, motivation, or trigger. Please think about what the weakest part is in pursuing your current goal.” Following the selfdiagnosis, users are presented with additional behavior-change techniques for the diagnosed problem domain.

Finally, the self-tracking methods were integrated into the revised prototype without any changes.

\subsection{User Study of the Revised Prototype}

To evaluate usability and usefulness of the revised prototype, a user study in which participants created or revised behavior change plans to their self-identified problems with the prototype was conducted.

\subsubsection{Method}

\section{Procedure}

Participants completed three one-on-one sessions one week apart. By design, the sessions were delivered by individuals with no formal clinical training to ensure their behavior did not contribute to potential effects of the session design. In session one, participants chose an issue to work toward and created their initial plan. Then, in sessions two and three, they were asked to reflect on the quality of their plan and revise it if necessary. In session two only, participants were taught the model and informed that the four techniques taught in session one were examples of each domain. Participants 
were asked to self-diagnosis the most problematic domain for them (i.e., is this a trigger, opportunity, motivation, or ability problem) and were then presented two additional techniques for the problem domain. In all sessions, participants were given a chance to either incorporate or ignore each technique.

\section{Participants}

Using mailing lists, recruitment emails were distributed graduate and college students at Arizona State University. Except for the age constraint to only recruit individuals 18 years or older, there were no further exclusion and inclusion criteria. Participants were a convenience sample ( $N=7 ; 5$ female and 2 male, with one dropout) of graduate students from Arizona State University. Ages ranged from 21 to 29 years of age. Participants received an Amazon gift card after each session ( $\$ 20, \$ 30$, and $\$ 40$, for a total $\$ 90)$.

\section{Measures and Analysis}

A survey consisting of 7-point Likert scale questions was used to examine session experience, satisfaction with their plans in the post-session surveys, and perceived success at achieving a goal during sessions two and three. A semi-structured interview was also conducted after each session based on participants' survey responses. A general inductive approach was utilized, whereby all recordings (both of the interviews and the sessions) were listened to and themes that arose were documented.

\subsubsection{Results}

\section{Perceived Usability}

Participants' response to the survey questions asking about overall experience at each session revealed that they considered the session work easy in general (Table 6). 
Specifically, participants were able to easily understand the concepts of "behavioral goals", "SMART goals", behavior change techniques provided, and meta-models. Consequently, they were able to work with those concepts, as shown in Table 7.

Table 6. Overall Session Experience

\begin{tabular}{|c|c|c|c|}
\hline \multirow[t]{2}{*}{ Question } & \multicolumn{3}{|c|}{ Mean (SD) } \\
\hline & Session 1 & Session 2 & Session 3 \\
\hline $\begin{array}{l}\text { Rate your overall experience with this session from Very } \\
\text { Negative to Very Positive. }\end{array}$ & $6.5(0.5)$ & $6.4(0.8)$ & $6.7(0.5)$ \\
\hline I think the session was difficult to follow. & $1.1(0.4)$ & $1.6(1.1)$ & $1.2(0.4)$ \\
\hline $\begin{array}{l}\text { I think the materials and instructions were clear and easy } \\
\text { to understand. }\end{array}$ & $6.6(0.7)$ & $6.4(1.1)$ & $6.8(0.4)$ \\
\hline
\end{tabular}

Table 7. Experience with Goal-setting

Question Mean (SD)

Experience in goal-setting

I was able to understand what to do immediately when asked to think $6.4(0.7)$ of behavioral goals.

It was easy to understand the concept of SMART goals.

$6.9(0.4)$

It was easy to establish SMART goal(s).

$6.4(0.7)$

Experience with behavior change techniques

It was easy to understand the technique of defining your inspiration.

Define your inspiration

Find the opportune/dangerous time and place

$6.2(1.2)$

Define a trigger

$6.0(1.7)$

Script critical action

$6.5(0.5)$

$5.6(1.7)$

I felt confident in generating ideas applying this technique.

Define your inspiration

$6.4(1.1)$

Find the opportune/dangerous time and place

$5.0(1.4)$

Define a trigger

$6.1(0.6)$

Script critical actions

$5.1(1.5)$

Experience with the meta-model

It was easy to understand the concept of the model.

$6.2(1.6)$

I was quite sure that the chosen one (Opportunity, Trigger, Ability, or Motivation) needs to be improved/enhanced most for my current goal

$6.5(0.5)$ 
pursuing, compared with the other three.

As illustrated in Table 8, the responses to the questions related with engagement reveal that participants had an engaged experience during the sessions overall. As shown in Table 9, participants chose high scores regarding their final plan compared to the sessions as revealed by questions examining their degree of satisfaction.

Table 8. Engagement with the Sessions

\begin{tabular}{lccc}
\hline \multicolumn{1}{c}{ Question } & \multicolumn{3}{c}{ Mean (SD) } \\
\cline { 2 - 4 } & Session 1 & Session 2 & Session 3 \\
\hline The tasks did not catch my interest. & $2.3(1.5)$ & $1.9(1.1)$ & $2.5(1.2)$ \\
$\begin{array}{l}\text { My experience with the session was } \\
\text { rewarding. }\end{array}$ & $5.8(0.9)$ & $6.1(1.1)$ & $6.3(0.5)$ \\
It was difficult to focus on the tasks. & $1.4(0.7)$ & $1.4(0.5)$ & $1.2(0.4)$ \\
\hline
\end{tabular}

Table 9. Satisfaction with Final Plan

\begin{tabular}{llll}
\hline \multirow{2}{*}{ Question } & \multicolumn{3}{c}{ Mean (SD) } \\
\cline { 2 - 4 } & Session 1 & Session 2 & Session 3 \\
\hline Overall, I am satisfied with my plan. & $6.5(0.5)$ & $6.9(0.4)$ & $6.7(0.5)$ \\
I am excited about carrying out my plan. & $6.6(0.5)$ & $6.4(0.8)$ & $6.7(0.5)$ \\
My plan fits my lifestyle well. & $5.9(1.0)$ & $6.1(0.9)$ & $6.5(0.5)$ \\
$\begin{array}{l}\text { I am completely satisfied with how I'm going to use } \\
\text { the techniques to achieve this goal. }\end{array}$ & $6.1(0.6)$ & $6.7(0.5)$ & $6.5(0.5)$ \\
$\begin{array}{l}\text { Carrying out the plan will be essential for me to solve } \\
\text { my problem. }\end{array}$ & $6.4(0.7)$ & $6.7(0.5)$ & 7.0 (0.0) \\
\hline
\end{tabular}

\section{Behavioral Plan Creation}

(1) Participant's chosen area of focus and SMART Goal

The majority (i.e., 71\%, n=5) of participants focused on work-related tasks (e.g., write a manuscript), while two focused on health (e.g., sleep or exercise more). The participants 
chose their issues to work toward either because there was a pressing need (i.e., P3, P4, $\left.\mathrm{P}_{5}, \mathrm{P} 6\right)$ or they had a fundamental concern (P1, P2, P7). Commonly, the issues were long lasting, from a couple of months to multiple years. Responses to perceived success (i.e., "Please rate how successful you were at achieving your goal") were on average 7.43 out of 10 in session two, and 8.67 out of 10 in session three.

Although the survey results indicated that participants felt it was easy to make SMART goals (e.g., for the question, "It was easy to establish SMART goal(s)", Mean $=6.4, \mathrm{SD}=$ 0.7), many individuals $(57 \%, \mathrm{n}=4)$ found that they were unsure how best to set both a specific and realistic goal. For example, P6 hesitated on the timescale for achieving her goal and P3 often had guests or dinner appointments with friends. Based on this, she said that she would write during the day but would not specify any further details.

(2) Using behavior change techniques

Participants appeared to demonstrate better use of the behavior change techniques presented in this study compared to the first user study with the initial prototype. However, participants still experienced difficulties grasping some of the subtleties of the exercise. Unlike the user study with the initial prototype, participants reported liking and actively using the behavior change techniques when creating their plans (e.g., P5, "It's good to have all of them, at once"). However, majority of participants did not understand how to develop a satisfactory trigger $(71.5 \%, \mathrm{n}=5)$ or script a critical action $(71.5 \% . \mathrm{n}=$ 5) during session one. Many participants set triggering times that were not at the time when they would engage in the activity, which is a requirement for a good trigger. $\mathrm{P}_{4}$, for example, set a notification on her mobile phone at $12 \mathrm{PM}$ to remind her to work at 2 PM. These small details were not grasped at first but started to become understood after one week of experience. 
As another issue in participants' applying behavior change techniques observed was resistance to trying new options but favoring their present lifestyles. For instance, $\mathrm{P} 4$ defined "home" as her "opportune place" for studying as she typically studies there and it was confortable for her. However, after failing to achieve her goal, she finally tested studying in the library and found this approach effective. P6 previously worked only at school, with an inclination of separating work and living places. In trying an alternative approach to work at home in the morning before going to school, she demonstrated her feeling of doubt about effectiveness of her idea. However, she eventually found the approach to be a useful strategy.

Participants' negative assessment of a particular technique before testing it likely prevented their active use of it. For instance, with the technique of defining an inspiration, majority of participants responded that their idea was only for some creative or special activity such as training for marathon. Although P6 generated such ideas in the session as a trigger, she did not stick a post-it note on her bathroom mirror because she believed that that the thought was prevalent and she was highly motivated.

It was also observed that the example provided for a technique greatly impacted how creative most individuals were with extending a technique to something personally applicable. Specifically, majority of participants used the triggering example (i.e., notification from the phone) as the only type of trigger. While this may be adequate for some participants, there were others who exhibited a creative reinterpretation. For example, P2's "trigger" to be more empathic and understanding of others was the pressure he felt from his ring when he shook hands with others. This example demonstrates the wide range of self-definition that can occur and also the difficulty with providing enough information to facilitate it. While some participants did "own" their 
behavior-change technique implementation, many simply used the example provided in the study.

\section{(3) Benefits of iteration}

In this study, there were improvements in perceived understanding on how to set a SMART goal and selecting a technique during sessions two and three compared to session one (which was reinforced by the self-reported success in achieving the goals). This effect seemed strongly influenced by the person's personal experience using the plans. Unlike the user study with the initial prototype, plans almost always $(86 \%, \mathrm{n}=6)$ became more elaborate and personalized to the person's daily life. For instance, $\mathrm{P} 7$ set her target time to go to bed differently for weekdays and weekends, which was not differentiated initially, but set based on her failure the previous weekend. $\mathrm{P} 4$ originally set a goal of "work for 2 hours" but during session two changed the goal to the more actionable goal of "practice speaking through presentation twice per day". Participants also presented more vivid descriptions on how they carried out their plans. For instance, while initially P6 was going to work "in the morning," this goal was changed to "after having breakfast and checking emails and news”.

Finally, majority of participants $(71 \%, \mathrm{n}=5)$ demonstrated far better understanding of the different techniques after some experience testing them. For instance, many participants did not understand the idea of scripting critical actions during session one. Further, some participants considered that there were no discrete actions between primary activities they cared about (e.g., actions between watching TV and starting to study) and some thought it was not necessary to be mindful of actions as they were able to easily stop one activity and start another. However, after testing the activity, they found it to be an essential and important technique. 


\subsubsection{Discussion and Conclusion}

Results indicate that participants created and revised their behavior change plans easily, and importantly, the findings suggest that the addition of a SMART goal and provision of behavior change techniques structured with the meta-model enabled participants to produce specific and strategic behavioral plans, which evolved through iteration. Compared with plans made in the user study with the initial prototype, plans in this user study have better quality in terms of specificity and integration of behavior change techniques.

However, the study had several limitations. A convenience sample of educated individuals was used, thus generalizability is limited. In addition, the study was run over two weeks. Finally, the researcher's presence in sessions and involvement in delivering materials may have biased the ideation of the individuals. However, a protocol with limited added "customization" support was intentionally created by the researcher to minimize this issue.

Overall, the improvements introduced for the second prototype appeared to facilitate creation of specific behavioral plan and iterative improvement between the sessions.

\subsection{Future Work}

Although the revised prototype led to improved user performance, several problems were found including difficulty in specifying details and limited understanding and creativity in working with behavior change techniques. Although these issues tended to be improved through iteration, they could cause users to cease the tutorial at the beginning in the real-life use setting (i.e., purely self-directed work without planned sessions with facilitators). A key future direction in improving the current tutorial and 
develop digital interactive materials is to better understand how to further facilitate the creative personalization of the techniques. Further research on facilitating increased creativity and the techniques is important for aiding individuals to develop effective personalized plans. For instance, providing more examples, particularly extreme examples of a technique, could be an effective strategy to expand users' creativity (Cao, Fleming, \& Burnett, 2011), particularly given participants' tendency of adopting provided examples in the user study. Based on the positive effect of participants' realization of the usefulness of a technique or how to apply it to their lifestyle, support that can foster socalled "reflection-on-action" or "reflection-in-action" (Schön, 1983) may lead to more effective iteration.

\subsection{Summary and Next Steps}

As an initial effort to help users' creation of behavioral plans, iterative design processes were conducted in which the initial prototype was designed and evaluated and the results of the evaluation led to a revised prototype. In the initial work for future development of interactive digital materials, the fundamental approaches with the low-fidelity prototypes (slide presentations with facilitators' presence) were explored. Overall, participants in the user study of the revised prototype had better quality plans compared with those from the initial prototype user study. Such a result supports the adequateness of designing interactive digital materials based on the current prototype.

In this dissertation study, two approaches in supporting users' self-experimentation for behavior change were envisioned: provision of interactive digital materials that support users' creation of behavioral plan, and toolkits that enable users' implementation of context-aware just-in-time (JIT) interventions. Each of these approaches led to the tutorial that guides users' plan creation with behavior change techniques and the 
GaLLaG toolkit that allows rapid prototyping of sensor-based interactive systems. In chapter five, a comparative experiment that was conducted to investigate if these supports can result in improvement of target issues is presented. This evaluation was considered necessary because effectiveness is the ultimate goal of issues and confirmation on it would further drive work for improving the current tools with confidence. 


\section{COMPARATIVE USER STUDY}

In the previous chapters, the GaLLaG tool for users' implementation of context-aware JIT interventions and the tutorial for behavioral plan composed of SMART goals, the action plan based on behavior change techniques, and self-tracking methods were described. To evaluate effectiveness of these supports in improving target issues, a comparative user study was conducted. In this chapter, the experiment design and study results are presented. Then, the results are discussed and future work considered necessary based on findings from this user study is presented.

\subsection{Overview}

The user study selected people's aim to achieve better sleep quality, as a theme in investigating the proposed two supports. Quality of sleep is an essential factor that affects individual's physical vitality, emotional balance, and productivity, and it is impacted by various lifestyles including bedtimes, diet, physical activity during the daytime, etc. (Lacks \& Rotert, 1986). Importantly, poor sleep quality is frequently found in people's daily life and various causes such as psychological disturbances, painful medical conditions, genetic factors, stress, age, physiological and cognitive arousal, etc. are known to be related. Researchers have also emphasized the importance of certain habits or environmental factors (commonly referred to as sleep hygiene), which may facilitate or hinder good quality sleep (Lacks \& Rotert, 1986).

To investigate effectiveness of the proposed two supports in people's improving sleep quality, a between-subject randomized trial was designed, which compared the proposed supports with a psycho-education control. The study compared the following three conditions: 
- Sleep hygiene alone (SH)

- Sleep hygiene + Self-experimentation for behavior change tutorial (SH-SBT)

- Sleep hygiene + Self-experimentation for behavior change tutorial + GaLLaG tool (SH-SBT-GaLLaG)

Sleep hygiene education was provided for all three conditions. Sleep hygiene is a prevalent treatment used for supporting individuals in improving their sleep quality and although it is often necessary, it is typically an insufficient component of an effective sleep strategy (Grandner, Jackson, Gooneratne, \& Patel, 2014; Lacks \& Rotert, 1986). Further, its inclusion also ensures any a priori knowledge about sleep that might have occurred between the intervention conditions and controls is minimized. Based on this, its inclusion even in control groups provides a particularly robust control condition.

All participants in the comparative study were asked to track their sleep with two tools provided as part of treatment. The first was a daily sleep diary implemented via a mobile phone application called "PACO" (www.pacoapp.com). In the application, participants enter answers to the following questions (ideally immediately after waking up for accuracy but data can be entered at any time during the day): (1) When you went to bed, (2) How long (in minutes) it took to fall asleep, (3) When you woke up, and (4) How much you are satisfied with your sleep (from 1 to 10, higher scores are better). PACO triggers a reminder inviting a user to answer the questions at a user-specified time. The other tool was a watch-style sleep sensor called Jawbone UP Move (www.jawbone.com). The tool collects data types including: (1) Time a user fell asleep, (2) Time a user woke up, (3) Number of times a user awoke during the sleep period, and (4) Total time length of sleep during the sleep period. The device is not completely automatic and thus users 
should switch the mode manually by pushing a button when they go to bed and wake up in the morning.

I hypothesized the SH-SBT and SH-SBT-GaLLaG conditions would improve sleep quality more than a sleep hygiene control over seven weeks. The primary outcome measures were the Pittsburgh Sleep Quality Inventory (PSQI)(Buysse, Reynolds, Monk, Berman, \& Kupfer, 1989) and daily sleep satisfaction that participants recorded using PACO.

\subsection{Method}

\subsubsection{Procedure}

Study participation lasted seven weeks after initial enrollment and consent obtainment (Figure 23). Participants attended five individual in-person sessions with the following durations: session 1 for 30 minutes and sessions 2 - 4 for 15 minutes for the SH condition and 1.5 hours for the SH-SBT and SH-SBT- GaLLaG conditions. Session 5 lasted 30 minutes for all conditions.

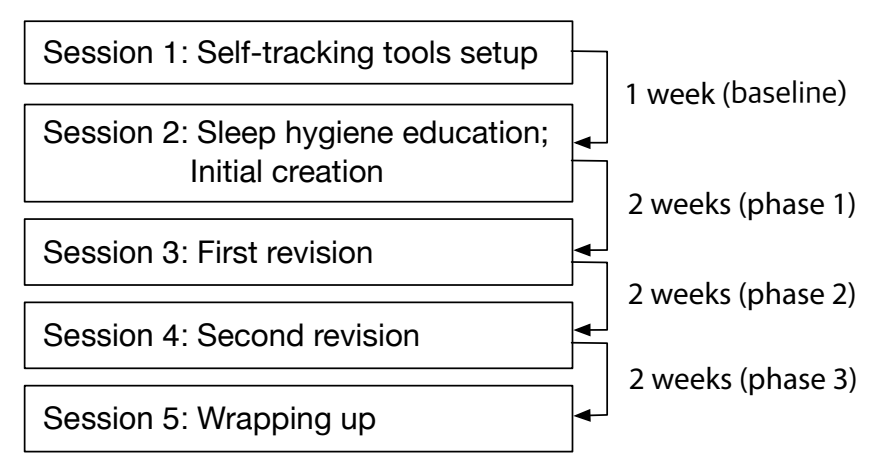

Figure 23. Study Procedure

In session one, participants of all conditions were given use instructions for the two selftracking tools, which they used until the end of the study. For the baseline data collection, participants were asked not to view the collected data until the next sessions. 
In session two, participants were asked to describe their main sleep issues, provided with sleep hygiene information, and asked to choose behaviors that they may want to eliminate or relieve for the coming two weeks at least. Then, each condition followed a different path, as described below:

- The SH-none condition made plans without any further support. Participants were asked to make their plans on how to attain the chosen behaviors including any details on what they would do.

- The SH-SBT condition made plans with the tutorial proposed (Section 4.3. The Revised Prototype). Participants set SMART goals with the chosen behaviors, and made plans for their attainment with lessons on behavior change techniques.

- The SH-SBT-GaLLaG condition participants followed all activities in the SH-SBT condition and also designed GaLLaG applications. For the application designing, they were first provided with an introduction to the concept of context-aware computing and the GaLLaG tool. Then, participants received application examples associated with behavior change techniques. For each example, a slideshow to describe the targeted behavior, the behavior change technique used, and a series of images showing the user interactions that could be implemented to realize the technique with the GaLLaG tool was presented.

Both of the SH-SBT and SH-SBT-GaLLaG groups were provided with worksheets to help them generate SMART goal and action plan ideas (Figure 24). The SH-SBT-GaLLaG group participants were asked to generate their ideas of the rules for implementation within the tool using sticky notes (Figure 25). This format was introduced to reinforce the rule-based logic and enable easy rule changes via post-it note movement. 


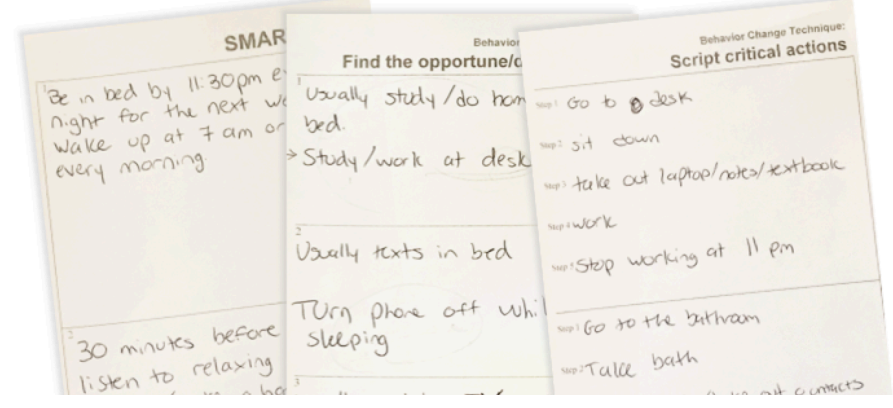

Figure 24. Use of Work Sheets

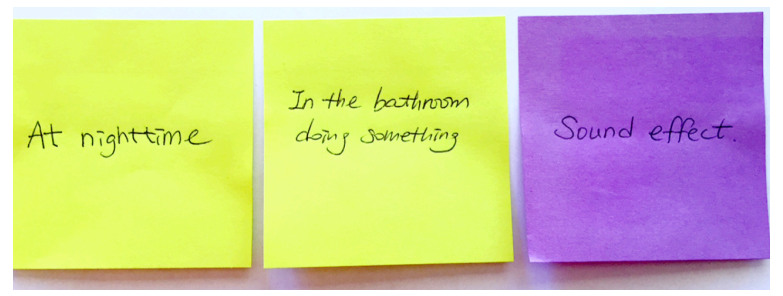

Figure 25. Example Ideation of GaLLaG Applications

All participants wrote a note describing their final plan and sent it to themselves via email as a personal reminder. They were encouraged to pursue set goals with the strategies developed prior to session three, complete the daily self-tracking, and freely check collected data whenever they wanted. For the SH-SBT-GaLLaG group, the researchers developed systems and visited their homes on a day agreed to by individuals.

Sessions three and four (the two revision sessions) began with a survey and interview investigating the past two-week sleep and plan implementation. If participants found it necessary, they could modify their goals and current main sleep issue they aimed to improve in the previous session. The SH group was encouraged to change or add any details to their plan. The SH-SBT and SH-SBT-GaLLaG condition participants learned additional behavior change techniques based on their self-diagnosis with the metamodel (Section 4.3. The Revised Prototype). For the SH-SBT-GaLLaG group condition, application examples corresponding to additionally provided behavior change 
techniques were provided. Given this new knowledge, participants generated ideas on how to apply them to their situation using the same approach as implemented in session two. All participants revised their plan description by merging the newly generated ideas into the previous plans. In session five, participants completed a wrap-up interview without plan revision.

\subsubsection{Measures}

\section{Screening Survey}

The screening survey examined the following four aspects: (1) Do participants have significant dissatisfaction toward their current sleep, not having have unmanaged sleeprelated disorders? (2) Do they have no circumstances (e.g., shift work) substantially influencing their sleep? (3) Do they have appropriate technologies for the self-tracking and GaLLaG applications installation (smartphone, WiFi at home)? (4) Do they have no trip planned or will they join a physical activity or behavior change program in the next seven weeks? Individual survey submissions were reviewed to determine their eligibility for the study. Sometimes, further inquiry to the participants was made via emails, primarily due to lack of information on the frequency of sleep issues or mobile phone models and operating systems. According to eligibility, emails notifying rejection (including reasons for rejection) or requesting to schedule the first session were distributed. The emails for scheduling also highlighted the scope of study and asked applicants to assess if the study seemed beneficial to their sleep issues, that is, if their main sleep issue could be improved with behavioral change. Before consenting to the first in-person session, eligibility was briefly checked again. 


\section{Sleep Quality}

To examine effectiveness of each intervention in resolving sleep problems, the Pittsburgh Sleep Quality Index (PSQI)(Buysse, et al., 1989; Grandner, et al., 2014) was used. The PSQI is a self-report questionnaire used in a wide range of sleep research including sleep interventions and consists of 19 questions. The questionnaire generates one global score (from o to 21) by summing the following seven "components" scores (from o to 3): (1) Subjective sleep quality, (2) Sleep latency (i.e., how long it takes to fall asleep), (3) Sleep duration, (4) Sleep efficiency (i.e., the percentage of time in bed that one is asleep), (5) Sleep disturbances, (6) Use of sleeping medication, and (7) Daytime dysfunction. Lower scores denote a healthier sleep quality. Daily self-tracked data on sleep patterns and subjective satisfaction were also collected (5.1. Overview). The small sample size, 9 for each condition (see 5.2.4 Participants for details), made it inappropriate to do significant testing. Thus, instead of it, I calculated descriptive statistics and then visualized the results to gain insights on possible change over time and any possible differences between groups.

\section{Behavior Change Plans}

To examine how participants created their behavior change plans, all sessions were video recorded and user-generated materials were collected including worksheets, GaLLaG application ideation using sticky notes, and notes of behavioral plans that were sent via email.

\subsubsection{Participants}

Targeted participants were adults (18 years and older) who had significant dissatisfaction with their current sleep but did not diagnosed sleep disorders as these 
would likely be too severe to expect improvement with the current experimental design. Using flyers and consent forms, participants were informed that a Jawbone UP Move device used during the study would be provided at the end of the study as compensation.

The screening survey was completed by 151 individuals. Seventy individuals were excluded for sleep issues beyond the scope of the study (e.g., not having noticeable sleep issue or having sleep apnea), mobile phone incompatibility, planning to take a trip or starting a physical activity programs, previous study participants, and couples (people who share their bedroom with someone else). Of the remaining 81 recruited participants, 41 individuals failed to join session one and 40 indicated their willingness to participate in the study and joined session one (Table 10).

Table 10. Follow-up of Screening Survey Respondents

\begin{tabular}{lc}
\hline \multicolumn{1}{c}{ Action } & $\begin{array}{c}\text { Number of } \\
\text { individuals }\end{array}$ \\
\hline $\begin{array}{l}\text { Rejected } \\
\text { Inappropriate sleep issues -- 49 }\end{array}$ & 70 \\
$\quad \begin{array}{l}\text { Incompatible mobile phones -- 6 } \\
\text { Plan of trip or start of physical activity programs -- } 4\end{array}$ & \\
$\quad$ Previous study participants -- 2 & \\
$\quad$ Couples (only in the additional recruitment) - 9 & 3 \\
Withdrawal* & 16 \\
No response to mail asking further questions for eligibility & 14 \\
No response to mail asking to schedule the first session & 4 \\
No show in the first session & 4 \\
No or wrong contact info & 40 \\
Consent & 151 \\
Total
\end{tabular}

*After querying on a scope and method of the study, possible subjects reported that they were not available during the study period (two respondents) or the study was not what they expected (one respondent). 
Among 40 participants, 13 were later excluded from the study for either failing to complete all sessions or sharing their bedroom with others. Thus, 27 participants (9 for each condition) were examined for main analysis (Figure 26).

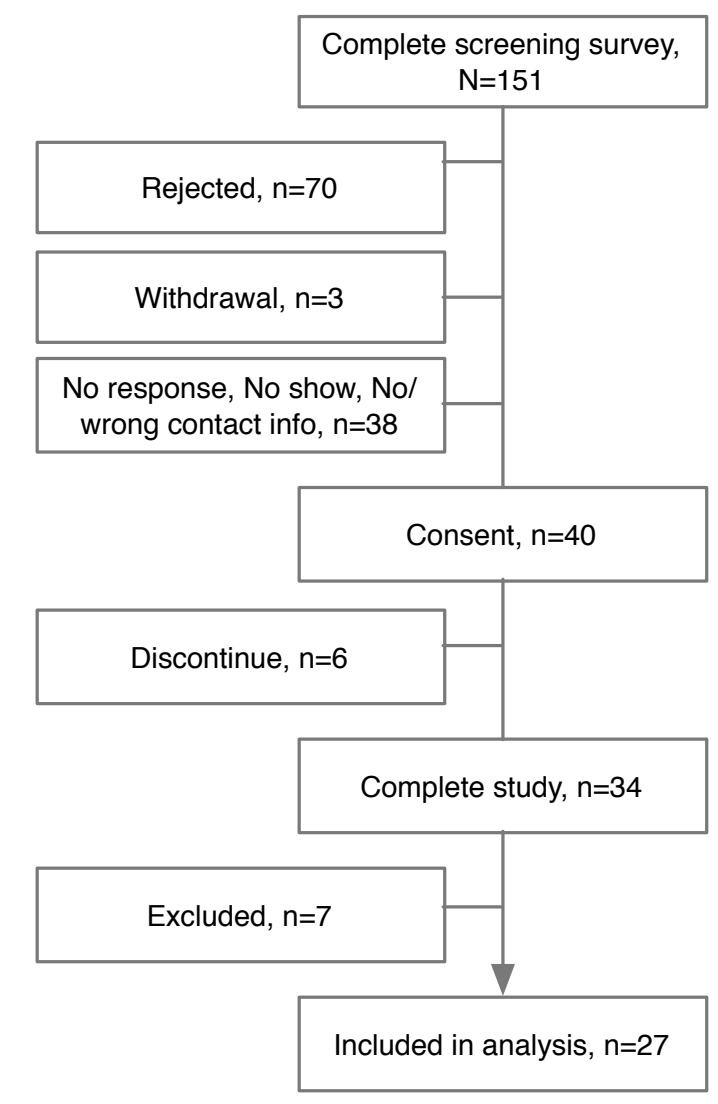

Figure 26. Participation Flow

Among the six dropouts, two did not attend a session or respond to email and phone contact. Two others had to leave town to manage personal emergency and the remaining two started a job that did not allow further participation.

\section{Demographics}

Of the 27 participants, there were 14 male participants and 13 female participants. The conditions were balanced in participant gender distribution. However, distribution of 
ages and occupations was not balanced over the conditions as illustrated in Tables 11 and 12.

Table 11. Age and Gender Distribution of Participants

\begin{tabular}{ccccccc}
\hline & \multicolumn{2}{c}{ SH } & \multicolumn{2}{c}{ SH-SBT } & \multicolumn{2}{c}{ SH-SBT-GaLLaG } \\
\cline { 2 - 7 } Age range & Male & Female & Male & Female & Male & Female \\
\hline $18-20$ & 1 & & & 1 & 2 & \\
$21-29$ & 4 & 3 & 3 & 2 & & 2 \\
$30-39$ & & 1 & 1 & 1 & 2 & \\
$40-49$ & & & & 1 & & 1 \\
$50-59$ & & & & & 1 & 1 \\
\hline
\end{tabular}

Table 12. Participants Occupations

\begin{tabular}{lccc}
\hline & SH & SH-SBT & SH-SBT-GaLLaG \\
\hline Employed for wages & - & 3 & 4 \\
Self-employed & 1 & - & - \\
Student & 8 & 6 & 4 \\
$\begin{array}{l}\text { Out of work, not currently } \\
\text { looking for work }\end{array}$ & - & - & 1 \\
\hline
\end{tabular}

\section{Sleep Issues}

Issues participants identified as relating to their sleep were classified to obtain a more detailed impression of their situations. Participants' sleep issues were classified into four groups: (1) Cannot fall asleep, (2) Do not keep a regular sleep schedule (stay up late and/or wake up late), (3) Wake up during the night (including waking up too early), and (4) Do not feel refreshed in the morning. Table 13 displays the numbers of participants assigned to each issue. 
Table 13. Sleep Issues and Numbers of Participants

\begin{tabular}{lcccc}
\hline \multicolumn{1}{c}{ Issue } & SH & SH-SBT & $\begin{array}{c}\text { SH-SBT- } \\
\text { GaLLaG }\end{array}$ & Total \\
\hline Cannot fall asleep & 2 & 3 & 1 & 6 \\
Not keep a regular sleep schedule & 3 & 4 & 2 & 9 \\
Wake up during the night & 3 & 2 & 4 & 10 \\
Not feel refreshed in the morning & 1 & o & 1 & 2 \\
\hline
\end{tabular}

\section{$5 \cdot 3$ Results}

\subsubsection{PSQI scores}

As illustrated in Table 14, all groups reduced their PSQI scores. For instance, the SHSBT-GaLLaG group's average score evaluating the baseline period was $10(\mathrm{SD}=1.6)$. However, the score evaluating the last two weeks of the study changed to $6.4(\mathrm{SD}=2.7)$. Figure 27 reveals a gradual decrease of the PSQI scores of the groups over the duration of the study (see the black line indicating the group average). As illustrated in Figure 28, all groups made improvement in most components. Notably, no or little substantial difference was found between the groups. It may be not plausible to observe a significant difference using this small a sample size and from a short-term experiment. However, the slightly larger decrease of the SH-SBT and SH-SBT-GaLLaG conditions compared to SH hints at the effectiveness of the two supports proposed.

Table 14. PSQI Score Comparison

\begin{tabular}{lccc}
\hline & SH & SH-SBT & SH-SBT-GaLLaG \\
\hline Session 2 & $8.2(2.0)$ & $9.4(2.4)$ & $10(1.6)$ \\
Session 5 & $5.8(3.5)$ & $5.3(3.9)$ & $6.4(2.7)$ \\
\hline
\end{tabular}




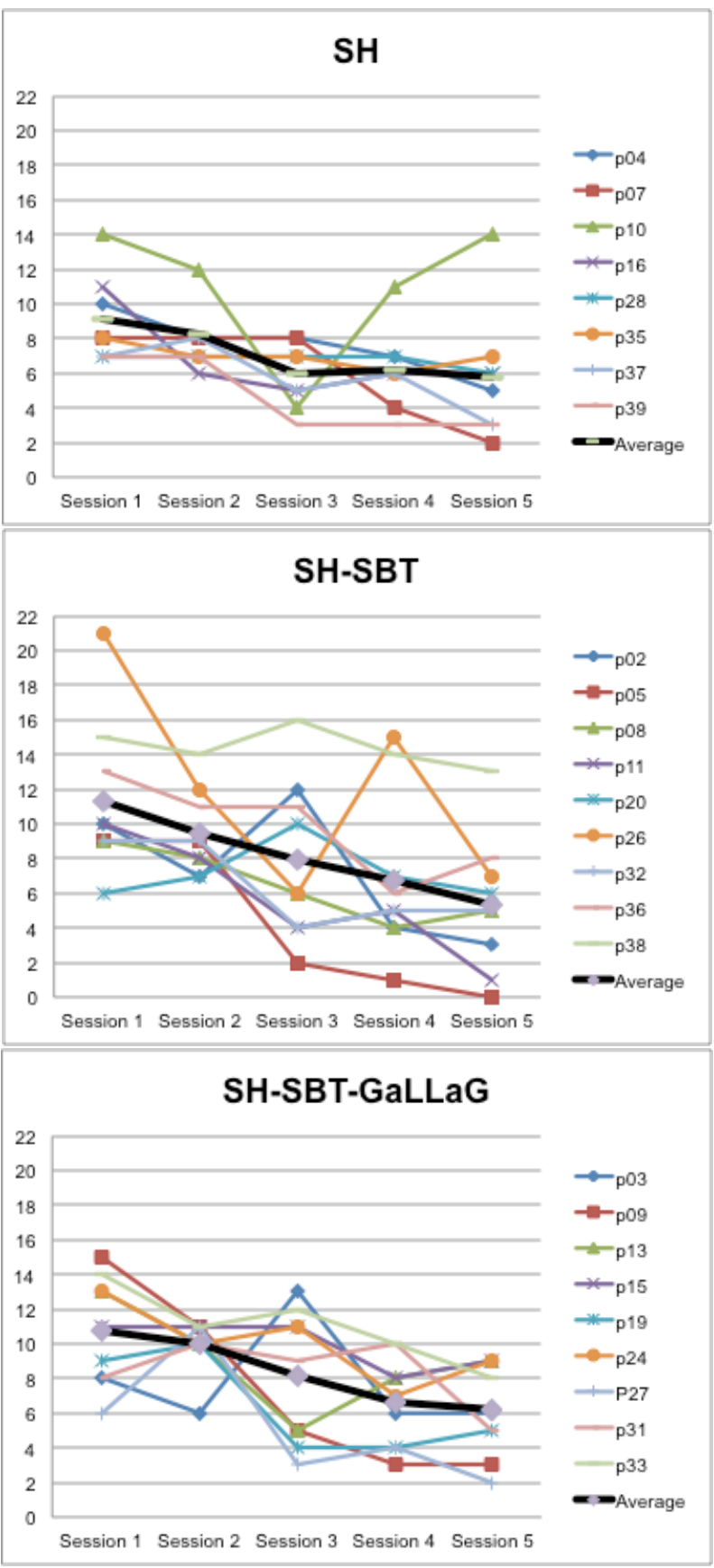

Figure 27. PSQI Score Trend 


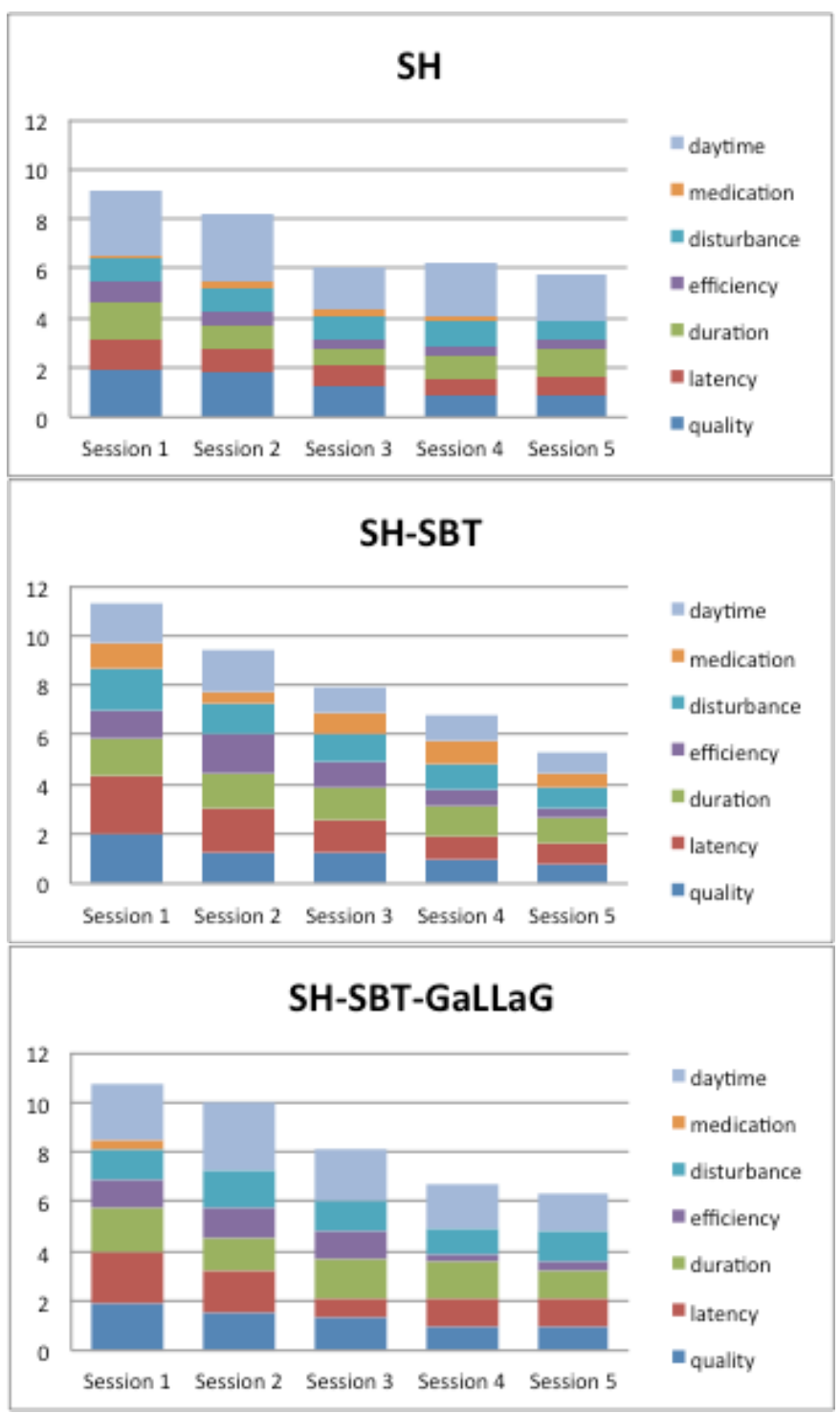

Figure 28. PSQI Components Scores

\subsubsection{Daily Sleep Satisfaction Ratings}

As illustrated in Table 15, all groups' daily satisfaction improved during phase three (i.e., between session four and five) compared with the baseline period. Figure 29 displaying the trend during the study indicates gradual increase over the study of the SH-SBT and SH-SBT-GaLLaG groups, which contrasts with the irregularity of the SH group. Similar 
to the PSQI result, a larger increase of the SH-SBT and SH-SBT-GaLLaG than the SH can be observed, although the difference is subtle.

Table 15. Daily Satisfaction Rating Change

\begin{tabular}{cccc}
\hline & SH & SH-SBT & SH-SBT-GaLLaG \\
\hline Baseline & $5.6(1.4)$ & $6.0(1.7)$ & $5.7(1.4)$ \\
Phase 3 & $5.8(1.2)$ & $6.8(1.2)$ & $6.5(1.5)$ \\
\hline
\end{tabular}

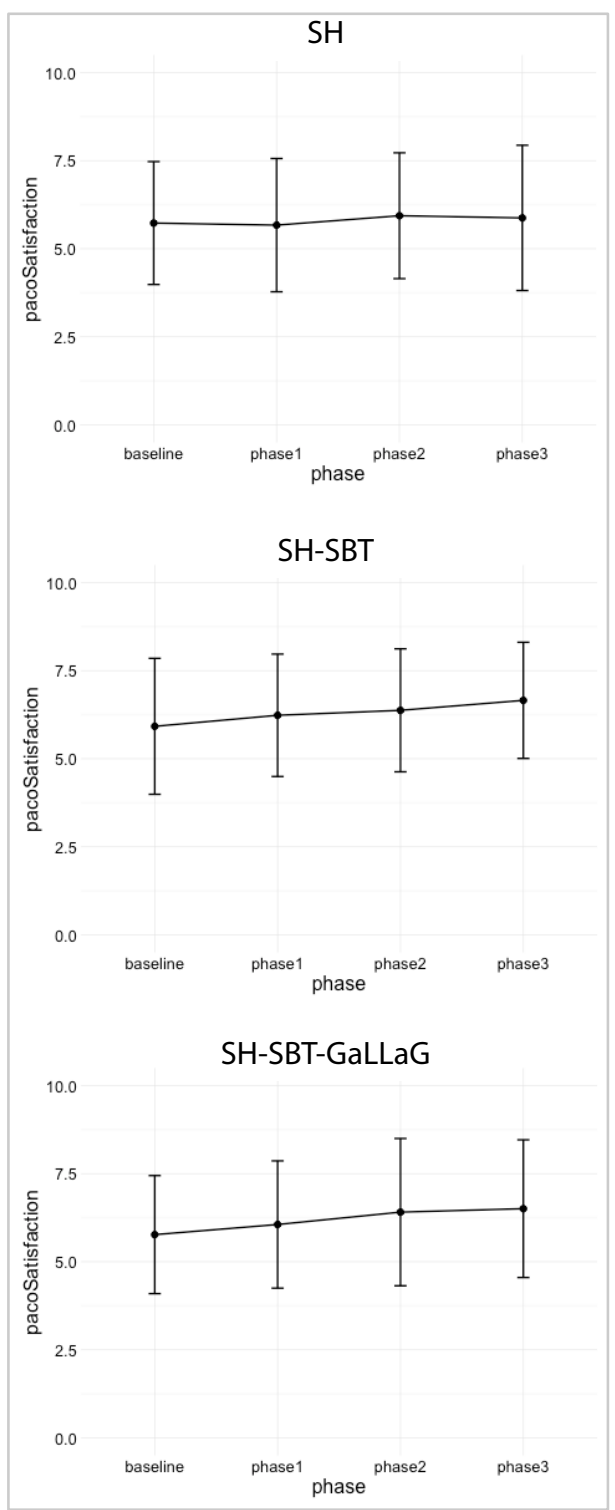

Figure 29. Daily Sleep Satisfaction Change 


\subsection{User Experiences}

In the previous section, the quantitative results of the comparative study did not reveal substantial effectiveness of the two supports proposed compared with the control condition, however, some potential was identified. To further evaluate participants' plan creation, written plans created by participants at each session and user interview responses were examined, in this section.

\subsubsection{Behavioral Plan}

Across all three conditions, participants selected similar behaviors based on sleep hygiene recommendations. Four behaviors were particularly popular amongst participants, and were as follows: "Adjusting/sticking to a sleep schedule" (16 participants), “Doing relaxing routines near bedtime” (15 participants), “Doing physical activity" (12 participants), and "Stopping stimulating activities near bedtime/Putting electronic devices away from the bed" (11 participants).

One key difference between the intervention and control groups was the specificity of the behavioral plans created. After choosing behaviors, participants made behavioral plans that defined how they would attain the behaviors. Results revealed that the SH-SBT and SH-SBT-GaLLaG conditions included more specificity in their plans than the SH control. An example can be found in the descriptions of how P28, Po8, and Pog defined times for the pursuit of "Adjusting/sticking to a sleep schedule" (Table 16).

Table 16. Exemplar Behavioral Plans in Pursuing "Adjusting/Sticking to a Sleep Schedule"

\begin{tabular}{lcc}
\hline P28 (SH), Session 2 & Po8 (SH-SBT), Session 2 & $\begin{array}{c}\text { Po9 (SH-SBT-GaLLaG), } \\
\text { Session } 2\end{array}$ \\
\hline Go to bed between 10 and 11 & Goals: Go to bed at 11 to 11:30 & Goals: No phone use near \\
\hline
\end{tabular}


PM, and wake up between 5:30 AM and $7 \mathrm{AM}$. In the morning, don't go back to bed.

Don't take a nap.

Avoid stimulants near bedtime.

\begin{tabular}{|c|c|}
\hline PM & bedtime. 10 PM. \\
\hline Wake up at 8:30 AM & Open curtains in morning. \\
\hline Plans: Set up calendar & Keep room light. \\
\hline $\begin{array}{l}\text { reminder at 10:30 PM to get } \\
\text { ready for bed }\end{array}$ & \multirow{5}{*}{$\begin{array}{l}\text { Plans: Remove chargers } \\
\text { earlier than } 10 \text { PM, and } \\
\text { transport all devices and } \\
\text { chargers into another room, } \\
\text { and finish up any tasks related } \\
\text { to the computer within the } \\
\text { guest room, and do not take } \\
\text { back any of the devices to your } \\
\text { room. }\end{array}$} \\
\hline $\begin{array}{l}\text { Turn off electronics at that } \\
\text { time }\end{array}$ & \\
\hline Start getting ready for bed & \\
\hline Relax before sleep & \\
\hline $\begin{array}{l}\text { Don't use electronics before } \\
\text { bed }\end{array}$ & \\
\hline Keep computer on desk & \multirow{2}{*}{$\begin{array}{l}\text { When waking up at } 8 \mathrm{AM} \text {, as a } \\
\text { first activity, walk to curtains } \\
\text { and open them to ensure a } \\
\text { steady flow of natural light. }\end{array}$} \\
\hline $\begin{array}{l}\text { Reward: if I go to bed on time } \\
\text { for a week, go shopping }\end{array}$ & \\
\hline
\end{tabular}

PM

Wake up at 8:30 AM

Plans: Set up calendar reminder at 10:30 PM to get ready for bed

Turn off electronics at that time

Relax before sleep

Don't use electronics before bed

Reward: if I go to bed on time for a week, go shopping bedtime. 10 PM

Open curtains in morning. Keep room light.

Plans: Remove chargers earlier than $10 \mathrm{PM}$, and transport all devices and anther room, to the computer within the guest room, and do not take back any of the devices to your om.

When waking up at $8 \mathrm{AM}$, as a and open them to ensure a steady flow of natural light.

This pattern continued in participants' revision in sessions three and four. In the $\mathrm{SH}$ control, participants did not typically change their plans. Instead, if they changed anything, they tried different sleep hygiene strategies. Specifically, in session three, six participants added one or two new behaviors and four participants removed one or two behaviors. In session four, three participants added one new behavior and four participants removed one behavior. In contrast, in the SH-SBT and SH-SBT-GaLLaG conditions, participants did not change their targeted sleep hygiene behaviors as much. During session three in the SH-SBT condition, three participants added one new behavior and two participants removed one. In session four, two participants added one new behavior and one participant removed one behavior. In addition, participants in the intervention conditions (SH-SBT and SH-SBT-GaLLaG) made changes to their behavioral plans including adding or removing behavior change techniques or modifying and further personalizing behavior change techniques, such as adding elements that had been poorly defined. For instance, Po8 (SH-SBT) specified items to avoid near bedtime (e.g. TV, movies, phone) in session three, which was only labeled "electronics" in session 
two. In session three, four of the SH condition participants did not make any changes to the details of their plans, while two of the SH-SBT and one of the SH-SBT-GaLLaG participants did not. In Session four, five participants in the SH condition did not change their plans, while two participants in the SH-SBT and all of the SH-SBT-GaLLaG participants changed their plans.

This exploratory work indicates that individuals across conditions that created more realistic, specific, and personalized plans had greater sleep improvements. However, the sample is too small to reach any firm conclusions. For example, $\mathrm{P}_{37}(\mathrm{SH})$ had more specific plans compared to others in the SH group. In the revisions, she gradually modified her plans to be more realistic and personalized (an initial 6:15 AM wake up time changed to 6:45 AM and $7 \mathrm{AM}$ and an initial plan of taking a warm bath with soothing music changed to reading or writing a journal). P37's PSQI score improved by 5 (baseline $=8$, phase $3=3$ ). In contrast, P10 realized the need to define activities during nighttime in session three and added "Make a relaxing bedtime routine" but included no further details. By session four, she reported that her bedtime routine did not feel more relaxing. In this scenario, the PSQI scores worsened (baseline $=12$, phase $3=14$ ).

\subsubsection{GaLLaG Applications}

Related to the SH-SBT-GaLLaG condition only, participants created one or more triggers for each target goal that they chose. For instance, Pog had a targeted behavior of establishing a bedtime routine and, as part of that developed a series of triggers focused on supporting their routine, particularly related to managing their smartphone. The first trigger was at 9pm, which sent a text message saying "charge the devices." At 10 PM if the smartphone was not being changed, music would play in the bedroom. Finally, if the phone was charged on time (meaning plugged in prior to 10 PM for 3 nights in a row) 
AND when a person opened a box of candy THEN happy music would play. If the phone had not been charged and the box opened then sad music played. Pog also created a trigger at 8 a to play happy music to invite them to open the blinds.

Participants also revised their plans as one might expect. New triggers were created as new targets were chosen. For example, P27 added a trigger to not drink coffee after 4 PM in session 3 after realizing that was important but not specified in session 2. Existing triggers were also modified. For example, P13 added a trigger to an existing one to support going to bed. Initially, only music played in the living room at 9:45 PM, but in session 3, another piece of music played in the bedroom 10 minutes later. To support waking up, initially, music played only in the bedroom at 4:30 AM, but in session 3, music played if she entered the kitchen in 30 minutes.

While the trial was too small to detect significantly different effects between conditions, I had hoped that the addition of the JIT intervention would have supported even greater sleep improvements, even just as a trend. That does not appear to be the case. One possible explanation for this lack of improvement may have been a misalignment between triggers and plans. Sometimes, no triggers were created for a behavioral goal. While most users created triggers for the majority of their targets, P33 included a trigger for only 1 among his 4 targets, and P24, for 2 targets among her 5 to 7 targets (depending on session).

Further, participants who increased their sleep satisfaction the most in the SH-SBT-JIT group appeared to have better alignment between their plans and triggers. For example, P24, who made only minimal sleep quality improvement, only incorporated application responses for one target behavior among six. For waking up, she designed her application to play peaceful music at 5:45 AM and switch to loud rock music at 6AM if 
she did not awake. For her other behavioral targets including drinking water, no working in bed, increased exercise, relaxation near bedtime, and no liquid after $9 \mathrm{PM}$, she did not create any triggers. In contrast, P27, who did display improved sleep quality, created triggers for most of his behavioral goals. For exercise in the morning, he designed his application to play music when he entered the kitchen and to help him eat smaller meals, he made the application play music when he entered the kitchen after work hours. For no coffee after $4 \mathrm{pm}$, he created a SMS reminder at $4 \mathrm{PM}$. For no phone use at nighttime, he placed two responses: sound play when he connected the phone to the charger, and sound played when he was around the kitchen after 10:30PM if the phone has not been charged. The only behavior he did not create a trigger for way going to bed between 10:30 and 11:30PM.

A second area of improvement for the JIT intervention involves the full use of contextaware computing. In particular, participants mostly developed time-based triggers, such as P15 sending himself an SMS at 11AM on Sunday, saying "Meal plan". Action-based conditions that involve use of sensors (e.g., when opening the refrigerator between 7 and $8 \mathrm{PM}$, play sound to invite preparation of snack/lunch for tomorrow) were limited, and, in fact, 3 users (P15, P31, and $\mathrm{P}_{33}$ ) did not use any triggers other than time-based ones. There are substantial drawbacks to using primarily time-based triggers. If a user has irregular daily schedules, it is likely that the triggers will arrive at inopportune times, and perhaps even at times when users are unavailable. Future work should likely focus on further supporting individuals in theorizing and developing more complex JIT state rules to trigger targeted behaviors.

Mostly participants found their system support beneficial, which can be summarized into three types of support. First, it broke continuation of mindlessly busy or idle state: P19, 'I 
usually spent long time using computer', P33, 'It kept me doing the goals', P31, 'Yeah, like if I was distracted, playing video game or working on the homework, it was nice to get that text message...and then I realize 'it's late...', P13, 'First of all...it helped me become aware of the time. Because I.. I was just like moving back to the day before, I didn't know what time it was...All right now it's time to start to prepare, start to relax...' Second, it reminded of forgotten goals. Third, it induced positive emotion, influencing good sleep or not related to it: P13, '(the music play when she comes home after work, which was designed to remind her about prep for the next day) not necessarily about snack/lunch prep. Now you're are at home... now be relaxed', Po3, 'I really liked the music when I open the closet, and on Friday mornings. Though I failed in reaching the exercise goal, it was just fun, good to hear.'

\subsection{Discussions}

Results of this study reveal a trend that all three conditions may have improved sleep quality over seven weeks, with some likelihood that the two interventions resulted in somewhat more improvement in sleep quality relative to the control group. Given the sample size, the results can be considered promising. However, the experiment had several limitations. The sample was small and participants' ages were unbalanced across conditions. In addition, the repeated in-person meetings may have affected users' behavior significantly, limiting the ability to claim that the same result would be gained with a parallel digital tool (though this was controlled for between conditions).

Nevertheless, the results provide several important insights on how to support people's self-experimentation for behavior change. First, the results highlight the value of selfexperimentation for the behavior change framework (setting goals, making action plans for attainment of the goals, and establishing self-tracking methods) as a starting point 
for supporting individuals in the self-creation and evaluation of personalized behavior change plans. Future researchers should consider building on the framework if they want more personalized behavior change to be supported.

Second, the results revealed better sleep quality improvement for individuals who created specific and personalized plans, and elaborated on them over time. The finding validates the usefulness of the features added in revising the initial tutorial prototype. They include providing guidance for setting goals following the concept of SMART goals, teaching behavior change techniques with materials enriched with principles and examples, and prompting assessment based on a meta-model in revising. Future work should consider providing additional support for better performance in those activities.

Third, this study confirms participants' capability of understanding the utility of contextaware computing and the fundamentals of the GaLLaG toolkit. However, limitations found in participants' application designs imply that users may require further support in creating JIT interventions with the GaLLaG tool. Future work should consider better integration of training materials and prompts to help individuals devise triggers that take into account not merely time but also actions (e.g., motion sensors, opening and closing doors) that, when combined, could be used to better infer if a person had the opportunity to engage in a target behavior and would be receptive to interacting with the system. In addition, further work to enable users' own construction of GaLLaG applications is essential. Likewise, more hands-on experience in their daily life may increase their creativity. 


\subsection{Conclusion}

The results of the comparative study highlight the value of self-experimentation for the behavior change framework proposed and the possibility of individuals' creating their own JIT interventions using the GaLLaG tool. Such findings contribute to the HCI research field working for people's behavior change, particularly with the vision of supporting more personalized behavior change technologies.

Future work should further explore refining this approach via implementation of automated support (e.g., interactive web materials). The goals of this automated support would be to help individuals devise specific, realistic, actionable, and personalized behavior change plans and provide them with better training on the concepts of opportunity and receptivity for helping individuals in designing their own JIT triggers using the GaLLaG tool. Another aspect to investigate further is development of a construction tool that allows users' own creation, involved in their daily activities and that may prompt creativity. 


\section{CONCLUSION}

\subsection{Summary}

Although it is widely recognized that people's quality of life and wellbeing is significantly influenced by their engagement in particular behaviors, failure in sustaining desired behavior seems prevalent. Driven by the importance of behavior change and the struggle to implement and maintain it, HCI has been increasingly exploring supportive strategies. In particular, advancement of ubiquitous and context-aware computing has led to a number of approaches. However, these concepts primarily consist of the provision of pre-fabricated solutions designed and implemented by experts. Given the plausible difference between individuals' personality and lifestyles, such directions are considered deficient in meeting the unique needs of the users and thus resulting in better improvement in attaining particular behaviors.

By pursuing approaches for users' acquisition of behavior change plans optimized to their needs, I demonstrated the benefit of taking advantage of users' personal knowledge about themselves in devising the plans. An alternative and complementary approach to enable more personalized and precise behavior change is likely to help individuals create and test their own behavior change plans. Existing practices for better lifestyles, such as the QS movement and the end-user development paradigm that has evolved as an important element of HCI, support the feasibility and usefulness of users creating personal behavior change plans.

In the development of supports to help users' creation and testing of behavior change plans, two different approaches were studied: (1) provision of interactive digital materials that support users' creation of behavioral plans incorporating behavior change 
techniques, and (2) provision of a tool that allows users' construction of JIT interventions employing context-aware technology. As initial work for the interactive digital materials that facilitate behavioral plan creation, a framework that defines three primary components of a behavioral plan was established. The components were: goals, action plans for the attainment of the goals, and self-tracking tools. A tutorial prototype that guides users' step-by-step to obtain plans composed of these three components was designed. Specifically, the tutorial facilitates creation of SMART goals in the goal setting and use of behavior change techniques in the action plan creation. Furthermore, the guidance leads user' reflection on their current plans in terms of four components that should exist for a behavior to occur, these are: opportunity, ability, motivation, and triggers. The user study investigating the prototype indicated usability of the tutorial, that is, participants were able to understand various concepts provided and complete tasks without any significant difficulties. In addition, participant-generated behavioral plans were satisfactory, that is, participants generally set goals that fit the concept of SMART goals, and created or revised plans incorporating behavior change techniques provided.

A context-aware JIT intervention tool that allows users to build sensor-based feedback systems within their home was created by adopting an existing platform, GaLLaG (Game as Life, Life as Game), which was developed by the research group, Motivational Environments (Burleson et al., 2009). The tool enables rapid prototyping of rule-based systems integrating simple sensor technology (magnetic sensors and PIR motions sensors) and media event components (sound plan, SMS, and appliance control). Such capability was chosen to allow easy learning and implementation of diverse applications. The user study conducted to evaluate its programming interface indicated that users 
could easily follow fundamental ideas on what could be realized with the GaLLaG tool and develop application ideas.

Development of the two supports described in the previous paragraph prompted a question of whether their use could result in better improvement in target issues compared with no use. To answer this question, a between-subject randomized trial was conducted, which compared three conditions in sleep quality improvement over seven weeks. All participants were asked to choose behaviors, based on sleep hygiene information commonly provided, that they would like to pursue to improve their sleep issue. The control group was provided no further supports and asked to make plans on how they w ould attain chosen behaviors including any details. Each treatment group received the corresponding support. Quantitative analysis results did not reveal significant difference between the conditions. Instead, a subtle inclination of greater improvement of the two treatment groups was observed. Examination of participantcreated behavioral plans revealed that specificity of plans and elaboration of details through revising was related with better sleep improvement. These findings indicate the usefulness of the tutorial proposed for user creation of behavioral plans and suggest a potential benefit of pursuing future work that adopts this framework in fostering userdriven behavior change plans. Conversely, results did not indicate effectiveness of involving GaLLaG applications compared with the behavioral plan only with the tutorial condition. Participants' limited employment of sensor-based triggers is considered related to such a result. 


\subsection{Contributions}

This dissertation study approaches the problem of supporting users' selfexperimentation for behavior change and provides contributions to users and HCI as described in the following section.

\subsubsection{Contributions to Users}

First, the framework of behavioral plan and tutorial that guides users' creation of plans based on behavior change techniques are proposed and can immediately be used by people who intend to improve their current lifestyles (e.g., QSers). The framework and tutorial are applicable to a wide range of issues such as taking medication regularly, doing more physical activity, quitting smoking, spending less money, being productive at work, etc.

Second, the meta-model proposed in this dissertation may serve as a classifier that people can use when they are exposed to many other behavior change techniques. By considering which of the four components in the meta-model this belongs to, a person may be able to better grasp core ideas of a newly found technique.

Third, this dissertation highlights the usefulness of JIT interventions and demonstrates how existing technologies can be integrated to implement more context-sensitive triggers. In addition, the GaLLaG tool may inspire people in the do-it-yourself culture to replicate the tool based on detailed information provided in this dissertation, and possibly expand the usage by adding newly available elements.

\subsubsection{Contributions to HCI}

First, this dissertation study highlights the need of expanding the scope of HCI with regard to helping people's effort for lifestyle improvement, which has been limited to the 
provision of pre-fabricated solutions and support of self-experimentation for discovery. As an alternative approach of such an existing trend, this study suggests selfexperimentation for behavior change and presents ideas on how to support this approach. Participants' sleep improvement in the final user study highlights the value of users' involvement in creating behavioral strategies in resolving target issues. This result may draw the attention of researchers in HCI, leading to further work based on the suggestions provided in this study to support users' self-experimentation for behavior change.

More specifically, support for users' creation of behavioral plan was suggested. In designing this support, several strategies were proposed, including the framework that defines core elements of a plan, combination of behavior change techniques for potentially effective plans for behavioral goal attainment, and use of a meta-model for self-diagnosis in revising. These ideas could be integrated in HCI researchers' work to help user-driven behavior change. In addition, this dissertation study reveals several problems in users' creating behavioral plans, including difficulty in specifying details and limited creativity in applying behavior change techniques.

In addition, support for implementing context-aware JIT interventions was suggested. With the GaLLaG tool, the idea of employing simple but robust technologies to create end-user development was implemented. This approach may inspire designers and developers who intend to develop end-user tools for context-aware applications. As previously discussed, limited creativity in generating GaLLaG application ideas was observed, which re-confirms existing study's assertion that there is a need to support users' design process. Support should not only provide tools for constructing designs but also help users develop rich and meaning design ideas. 


\subsection{Limitations and Future Work}

The comparative user study conducted to investigate the usefulness of the two proposed interventions compared to the controlled condition included a relatively small number of participants (nine participants in each condition). The small sample size made it inappropriate to use statistical methods to find significant differences. Another limitation of the comparative study is that a researcher intervened in participants' experiences. To complement the limited functionality of the present prototypes, a researcher administered participants' work with the proposed tutorial and implemented GaLLaG applications. To validate the value of self-experimentation, future work should include a user study with a larger sample size and in which participants have full autonomy and capability in creating and revising behavior change plans. This future work would require advancement of the current prototypes, that is: first, the current tutorial for creation of behavioral plans should be transformed into interactive tools; and second, the GaLLaG tool should be further developed so end-users can program GaLLaG applications and install all required hardware (sensors, speakers, etc.).

In developing the current prototypes to be tools allowing end-users' individual work, approaches to enhance users' creativity should be considered. In developing interactive digital tools for creation of behavioral plan, two types of augmentation leveraging information technology seem plausible, and are as follows: (1) support in authoring plans and (2) support in assessing plans. Existing strategies to enhance users' creativity such as using templates, auto-completion or suggestion, or a wizard could be incorporated for easy generation of plans with rich details (Shneiderman, 2000). Users' assessment of the appropriateness of created plans (e.g., "Is this goal realistic?”) could be enhanced with existing approaches such as provision of an expert system (Velicer et al., 1993) and 
support of user reflection based on collected behavioral data (M. Lee, Kim, Forlizzi, \& Kiesler, 2015). In addition, the current programming interface of the GaLLaG requires expansion of programming functionality, being mindful that increased complexity could immediately repel users. 


\section{REFERENCES}

Alexander, C. (1964). Notes on the Synthesis of Form. Harvard University Press.

Arroyo, E., Bonanni, L., \& Selker, T. (2005). Waterbot: exploring feedback and persuasive techniques at the sink. In Proceedings of the SIGCHI conference on Human factors in computing systems (pp. 631--639).

Artinger, F., Petersen, M., Gigerenzer, G., \& Weibler, J. (2015). Heuristics as adaptive decision strategies in management. Journal of Organizational Behavior, 36(S1), S33-S52.

Attin, T., \& Hornecker, E. (2005). Tooth brushing and oral health: how frequently and when should tooth brushing be performed?. Oral health \& preventive dentistry, 3(3).

Bandura, A. (1986). Social foundations of thought and action: A social cognitive theory.. Prentice-Hall, Inc.

Bandura, A. (1991). Self-regulation of motivation through anticipatory and self-reactive mechanisms. In Perspectives on motivation: Nebraska symposium on motivation (pp. 69--164).

Bandura, A. (2001). Social cognitive theory: An agentic perspective. Annual review of psychology, 52(1), 1--26.

Bang, M., Gustafsson, A., \& Katzeff, C. (2007). Promoting new patterns in household energy consumption with pervasive learning games. Persuasive Technology, 55-63.

Baumeister, R. F., Heatherton, T. F., \& Tice, D. M. (1994). Losing Control: How and Why People Fail at Self-Regulation. Academic Press.

Baumeister, R. F., Vohs, K. D., \& Tice, D. M. (2007). The strength model of self-control. Current directions in psychological science, 16(6), 351--355.

Beckmann, C., \& Dey, A. (2003). Siteview: Tangibly programming active environments with predictive visualization. In adjunct Proceedings of UbiComp (pp. 167-168).

Bonanni, L., Arroyo, E., Lee, C. H., \& Selker, T. (2005). Smart sinks: real-world opportunities for context-aware interaction. In CHI'O5 Extended Abstracts on Human Factors in Computing Systems (pp. 1232-1235). ACM.

Britton, B. K., \& Glynn, S. M. (1989). Mental management and creativity. In Handbook of creativity (pp. 429-440). Springer US.

Britton, B. K., \& Tesser, A. (1991). Effects of time-management practices on college grades. Journal of educational psychology, 83(3), 405.

Buechley, L. (2009). DIY for CHI : Methods, Communities , and Values of Reuse and Customization. In CHI'o9 Extended Abstracts on Human Factors in Computing Systems (pp. 4823-4826). ACM. 
Burleson, W., Ruffenach, C., Jensen, C., Bandaru, U. K., \& Muldner, K. (2009). Game as life--life as game. In Proceedings of the 8th International Conference on Interaction Design and Children (pp. 272-273). ACM.

Buysse, D. J., Reynolds, C. F., Monk, T. H., Berman, S. R., \& Kupfer, D. J. (1989). The Pittsburgh Sleep Quality Index: a new instrument for psychiatric practice and research. Psychiatry research, 28(2), 193--213.

Cao, J., Fleming, S. D., \& Burnett, M. M. (2011). An exploration of design opportunities for" gardening" end-user programmers' ideas.. In VL/HCC (pp. 35--42).

Castiglioni, A. (1931). Life and work of Sanctorius. Medical Life, 38, 729-785.

Chin, J. S., Callaghan, V., \& Clarke, G. (2006). An end-user programming paradigm for pervasive computing applications. In Pervasive Services, 2006 ACS/IEEE International Conference on (pp. 325--328).

Choe, E. K., Lee, N. B., Lee, B., Pratt, W., \& Kientz, J. A. (2014). Understanding quantified-selfers' practices in collecting and exploring personal data. In Proceedings of the 32nd annual ACM conference on Human factors in computing systems (pp. 1143-1152).

Couger, J. D. (1996). Creativity \& innovation in information systems organizations. Boyd \& Fraser Publishing Company.

Cypher, A. \& Halbert, D. C. (1993). Watch what I do: programming by demonstration. MIT press.

D'Zurilla, T. J. \& Goldfried, M. R. (1971). Problem solving and behavior modification.. Journal of abnormal psychology, 78(1), 107.

D’Zurilla, T. J., and Nezu, A. M., (2010). Problem-solving therapy. In K. S. Dobson (Ed.), Handbook of cognitive-behavioral therapies, 3rd edition. Guilford Press.

Dalal, S., Alwan, M., Seifrafi, R., Kell, S., \& Brown, D. (2005). A rule-based approach to the analysis of elders activity data: Detection of health and possible emergency conditions. In AAAI Fall 2005 Symposium (pp. 2545--2552).

De Sá, M., \& Carriço, L. (2009). A mobile tool for in-situ prototyping. In Proceedings of the 11th International Conference on Human-Computer Interaction with Mobile Devices and Services (p. 20). ACM.

de Ridder, Denise T. D. and de Wit, John B. F. (2006). Self-regulation in health behavior: Concepts, theories, and central issues. In de Ridder, Denise T. D. and de Wit, John B. F. (Eds.), Self-regulation in health behavior (pp. 1-23). John Wiley \& Sons Ltd.

Dey, A. K., Sohn, T., Streng, S., \& Kodama, J. (2006). iCAP: Interactive prototyping of context-aware applications. In International Conference on Pervasive Computing (pp. 254-271). Springer Berlin Heidelberg.

Dorst, K. (2003). The problem of design problems. Expertise in design, 135-147. 
Dresslar, F. B. (1892). Some influences which affect the rapidity of voluntary movements. The American Journal of Psychology, 4(4), 514--527.

Dunbar-Jacob, J., \& Mortimer-Stephens, M. (2001). Treatment adherence in chronic disease. Journal of clinical epidemiology, 54(12), S57-S60.

Edinger, J. D., Wohlgemuth, W. K., Radtke, R. A., Marsh, G. R., \& Quillian, R. E. (2001). Cognitive behavioral therapy for treatment of chronic primary insomnia: a randomized controlled trial. Jama, 285(14), 1856-1864.

Ertas, A., \& Jones, J. C. (1996). The engineering design process. New York: Wiley.

Fava, G. A., Rafanelli, C., Grandi, S., Conti, S., \& Belluardo, P. (1998). Prevention of recurrent depression with cognitive behavioral therapy: preliminary findings. Archives of general psychiatry, 55(9), 816-820.

Fischer, G. (1993). Beyond Human Computer Interaction: Designing Useful and Usable Computational Environments. In People and Computers Viii: Proceedings of the HCI '93 Conference (pp. 17--31). Cambridge, UK: Cambridge University Press.

Fischer, G. \& Scharff, E. (2000). Meta-design: design for designers. In Proceedings of the 3 rd conference on Designing interactive systems: processes, practices, methods, and techniques (pp. 396--405).

Fischer, G. (2007). Meta-design: expanding boundaries and redistributing control in design. In IFIP Conference on Human-Computer Interaction (pp. 193--206).

Fisher, W. W., Piazza, C. C., \& Roane, H. S. (Eds.). (2011). Handbook of applied behavior analysis. Guilford Press.

Fogg, B. J. (2002). Persuasive Technology: Using Computers to Change What We Think and Do. Morgan Kaufmann.

Franz, M. J., VanWormer, J. J., Crain, A. L., Boucher, J. L., Histon, T., Caplan, W., ... \& Pronk, N. P. (2007). Weight-loss outcomes: a systematic review and meta-analysis of weight-loss clinical trials with a minimum 1-year follow-up. Journal of the American Dietetic Association, 107(10), 1755-1767.

Funnell, M. M., Brown, T. L., Childs, B. P., Haas, L. B., Hosey, G. M., Jensen, B., Maryniuk, M., Peyrot, M., Piette, J. D., Reader, D., \& others (2008). National standards for diabetes self-management education. Diabetes care, 31(Supplement 1), S87--S94.

García-Herranz, M., Haya, P. A., \& Alamán, X. (2010). Towards a Ubiquitous End-User Programming System for Smart Spaces.. J. UCS, 16(12), 1633--1649.

Grandner, M. A., Jackson, N., Gooneratne, N. S., \& Patel, N. P. (2014). The development of a questionnaire to assess sleep-related practices, beliefs, and attitudes. Behavioral sleep medicine, 12(2), 123--142.

Greenbaum, J. M., \& Kyng, M. (Eds.). (1991). Design at work: Cooperative design of computer systems. Routledge. 
Grilo, C. M., \& Masheb, R. M. (2005). A randomized controlled comparison of guided self-help cognitive behavioral therapy and behavioral weight loss for binge eating disorder. Behaviour Research and Therapy, 43(11), 1509-1525.

Hekler, E. B., Klasnja, P., Froehlich, J. E., \& Buman, M. P. (2013a). Mind the theoretical gap: interpreting, using, and developing behavioral theory in HCI research. In Proceedings of the SIGCHI Conference on Human Factors in Computing Systems (pp. 3307--3316).

Hekler, E. B., Michie, S., Pavel, M., Rivera, D. E., Collins, L. M., Jimison, H. B., ... \& Spruijt-Metz, D. (2016b). Advancing Models and Theories for Digital Behavior Change Interventions. American Journal of Preventive Medicine.

Horn, M. S., Solovey, E. T., Crouser, R. J., \& Jacob, R. J. (2009). Comparing the use of tangible and graphical programming languages for informal science education. In Proceedings of the SIGCHI Conference on Human Factors in Computing Systems (pp. 975-984). ACM.

Humble, J. and Crabtree, A. (2003). "Playing with the Bits" User-configuration of Ubiquitous Domestic Environments. Proc. UbiComp 2003, Springer, 256-263

Intille, S. S. (2002). Designing a home of the future. IEEE pervasive computing, 1(2), 7682.

Jafarinaimi, N., Forlizzi, J., Hurst, A., \& Zimmerman, J. (2005). Breakaway: an ambient display designed to change human behavior. In CHI'O5 extended abstracts on Human factors in computing systems (pp. 1945-1948). ACM.

Karkar, R., Zia, J., Vilardaga, R., Mishra, S. R., Fogarty, J., Munson, S. A., \& Kientz, J. A. (2015). A framework for self-experimentation in personalized health. Journal of the American Medical Informatics Association, ocv150.

Kendall, P. C., \& Southam-Gerow, M. A. (1996). Long-term follow-up of a cognitivebehavioral therapy for anxiety-disordered youth. Journal of consulting and clinical psychology, 64(4), 724 .

King, A. C., Hekler, E. B., Grieco, L. A., Winter, S. J., Sheats, J. L., Buman, M. P., ... \& Cirimele, J. (2013). Harnessing different motivational frames via mobile phones to promote daily physical activity and reduce sedentary behavior in aging adults. PloS one, 8(4), e62613.

Klasnja, P. V., Consolvo, S., McDonald, D. W., Landay, J. A., \& Pratt, W. (2009). Using mobile \& personal sensing technologies to support health behavior change in everyday life: lessons learned. In AMIA.

Ko, A. J., Myers, B. A., \& Aung, H. H. (2004). Six learning barriers in end-user programming systems. In Visual Languages and Human Centric Computing, 2004 IEEE Symposium on (pp. 199--206). 
Kobsa, A., Chen, Y., \& Wang, T. (2012). Discovering personal behavioral rules in a health management system. In 2012 6th International Conference on Pervasive Computing Technologies for Healthcare (PervasiveHealth) and Workshops (pp. 224-227). IEEE.

Konečni, V. J. (2008). Does music induce emotion? A theoretical and methodological analysis.. Psychology of Aesthetics, Creativity, and the Arts, 2(2), 115.

Lacks, P. \& Rotert, M. (1986). Knowledge and practice of sleep hygiene techniques in insomniacs and good sleepers. Behaviour Research and Therapy, 24(3), 365--368.

Latham, G. P. (2003). Goal Setting:: A Five-Step Approach to Behavior Change. Organizational Dynamics, 32(3), 309--318.

Lee, J., Garduño, L., Walker, E., \& Burleson, W. (2013). A tangible programming tool for creation of context-aware applications. In Proceedings of the 2013 ACM international joint conference on Pervasive and ubiquitous computing (pp. 391-400). ACM.

Lee, J., Walker, E., Burleson, W., \& Hekler, E. B. (2014b). Programming tool of contextaware applications for behavior change. In Proceedings of the 2014 ACM International Joint Conference on Pervasive and Ubiquitous Computing: Adjunct Publication (pp. 91-94).

Lee, M. K., Kim, J., Forlizzi, J., \& Kiesler, S. (2015). Personalization revisited: a reflective approach helps people better personalize health services and motivates them to increase physical activity. In Proceedings of the 2015 ACM International Joint Conference on Pervasive and Ubiquitous Computing (pp. 743-754). ACM.

Li, I., Dey, A. K., \& Forlizzi, J. (2011). Understanding my data, myself: supporting selfreflection with ubicomp technologies. In Proceedings of the 13th international conference on Ubiquitous computing (pp. 405-414). ACM.

Liikkanen, L. A., \& Perttula, M. (2009). Exploring problem decomposition in conceptual design among novice designers. Design studies, 30(1), 38-59.

Lieberman, H., Paternò, F., Klann, M., \& Wulf, V. (2006). End-user development: An emerging paradigm. In End user development (pp. 1-8). Springer Netherlands.

Lindner, H., Menzies, D., Kelly, J., Taylor, S., \& Shearer, M. (2003). Coaching for behaviour change in chronic disease: a review of the literature and the implications for coaching as a self-management intervention. Australian Journal of Primary Health, 9(3), 177--185.

Locke, E. A. \& Latham, G. P. (2002). Building a practically useful theory of goal setting and task motivation: A 35-year odyssey.. American psychologist, 57(9), 705.

Lorig, K. (2001). Patient education: a practical approach. Sage.

Lozano, C., Burleson, W., Ravishankar, V., Lee, J., Muldner, K., \& Mahoney, D. (2014). ASSISTING WITH DRESSING ACTIVITIES FOR PEOPLE WITH DEMENTIA VIA ADAPTIVE PROMPTING. Alzheimer's \& Dementia: The Journal of the Alzheimer's Association, 10(4), P443. 
Lyytinen, K., \& Yoo, Y. (2002). Ubiquitous computing. Communications of the ACM, 45(12), 63-96.

Maitland, J. \& Chalmers, M. (2010). Self-monitoring, self-awareness, and selfdetermination in cardiac rehabilitation. In Proceedings of the SIGCHI Conference on Human Factors in Computing Systems (pp. 1213--1222).

Manimala, M. J. (1992). Entrepreneurial heuristics: A comparison between high PI (pioneering-innovative) and low PI ventures. Journal of Business Venturing, 7 (pp. 477504).

Michie, S., Abraham, C., Eccles, M. P., Francis, J. J., Hardeman, W., \& Johnston, M. (2011a). Strengthening evaluation and implementation by specifying components of behaviour change interventions: a study protocol. Implementation Science, 6(1), 1.

Michie, S., van Stralen, M. M., \& West, R. (2011b). The behaviour change wheel: a new method for characterising and designing behaviour change interventions.

Implementation Science, 6(1), 1.

Michie, S., Richardson, M., Johnston, M., Abraham, C., Francis, J., Hardeman, W., Eccles, M. P., Cane, J., \& Wood, C. E. (2013). The behavior change technique taxonomy (v1) of 93 hierarchically clustered techniques: building an international consensus for the reporting of behavior change interventions. Annals of behavioral medicine, 46(1), 81--95.

Modugno, F., Corbett, A. T., \& Myers, B. A. (1997). Graphical representation of programs in a demonstrational visual shell-an empirical evaluation. ACM Transactions on Computer-Human Interaction (TOCHI), 4(3), 276-308.

Muraven, M., \& Baumeister, R. F. (2000). Self-regulation and depletion of limited resources: Does self-control resemble a muscle?. Psychological bulletin, 126(2), 247.

Nahum-Shani, I., Hekler, E. B., \& Spruijt-Metz, D. (2015). Building health behavior models to guide the development of just-in-time adaptive interventions: A pragmatic framework. Health Psychology, 34(S), 1209.

Nardi, B. A. (1993). A Small Matter of Programming: Perspectives on End User Programming. Cambridge, MA: MIT Press.

Nawyn, J., Intille, S., \& Larson, K. (2006). Embedding behavior modification strategies into a consumer electronic device: a case study. In UbiComp 2006: Ubiquitous Computing (pp. 297-314). Springer Berlin Heidelberg.

Nelson, M. E., Rejeski, W. J., Blair, S. N., Duncan, P. W., Judge, J. O., King, A. C., Macera, C. A., \& Castaneda-Sceppa, C. (2007). Physical activity and public health in older adults: recommendation from the American College of Sports Medicine and the American Heart Association. Circulation, 116(9), 1094.

Neuringer, A. (1981). Self-experimentation: A call for change. Behaviorism, 9(1), 79--94.

Newman, M. W., Lauterbach, D., Munson, S. A., Resnick, P., \& Morris, M. E. (2011). It's not that i don't have problems, i'm just not putting them on facebook: challenges and 
opportunities in using online social networks for health. In Proceedings of the ACM 2011 conference on Computer supported cooperative work (pp. 341-350). ACM.

Newell, A., \& Simon, H. A. (1972). Human problem solving (Vol. 104, No. 9). Englewood Cliffs, NJ: Prentice-Hall.

Nezu, A. M., Nezu, C. M., \& D'Zurilla, T. (2012). Problem-solving therapy: A treatment manual. Springer Publishing Company.

Oksman, V. \& Rautiainen, P. (2003). " Perhaps it is a Body Part": How the Mobile Phone Became an Organic Part of the Everyday Lives of Finnish Children and Teenagers. Machines that become us: The social context of communication technology, 293--308.

Pierce, D. (2012). Problem solving therapy: Use and effectiveness in general practice. Australian family physician, 41(9), 676.

Porter, T., \& Ronit, K. (2006). Self-regulation as policy process: The multiple and crisscrossing stages of private rule-making. Policy Sciences, 39(1), 41-72.

Powell, S. G., \& Barker, K. R. (2008). Management Science: The Art Of Modeling With Spreadsheets. John Wiley \& Sons.

Räisänen, T., Oinas-kukkonen, H., \& Pahnila, S. (2008). Finding Kairos in Quitting Smoking : Smokers ' Perceptions of Warning Pictures. In Persuasive Technology (pp. 254-257). Springer Berlin Heidelberg.

Reitman, W. (1965). Cognition and thought. New York: Wiley.

Resnick, M., Bruckman, A., \& Martin, F. (1996). Pianos not stereos: Creating computational construction kits. interactions, 3(5), 40-50.

Resnick, M., Myers, B., Nakakoji, K., Shneiderman, B., Pausch, R., Selker, T., \& Eisenberg, M. (2005). Design principles for tools to support creative thinking.

Rittel, H. W., \& Webber, M. M. (1973). Dilemmas in a general theory of planning. Policy Sciences, 4(2), 155-169.

Roberts, S., \& Neuringer, A. (1998). Self-Experimentation. In K. A. Lattal \& M. Perrone (Eds.), Handbook of Research Methods in Human Operant Behavior. New York: Plenum,

Rosenbaum, M. (1980). A schedule for assessing self-control behaviors: Preliminary findings. Behavior therapy, 11(1), 109--121.

Schön, D. A. (1983). The reflective practitioner: How professionals think in action. Basic books.

Schulman, D., \& Bickmore, T. (2009). Persuading users through counseling dialogue with a conversational agent. In Proceedings of the 4th international conference on persuasive technology (p. 25). ACM. 
Seifert, J., Pfleging, B., del Carmen Valderrama Bahamóndez, E., Hermes, M., Rukzio, E., \& Schmidt, A. (2011). Mobidev: a tool for creating apps on mobile phones. In Proceedings of the 13th International Conference on Human Computer Interaction with Mobile Devices and Services (pp. 109-112). ACM.

Sheeran, P. (2002). Intention-behavior relations: A conceptual and empirical review. European review of social psychology, 12(1), 1-36.

Shneiderman, B. (2000). Creating creativity: user interfaces for supporting innovation. ACM Transactions on Computer-Human Interaction (TOCHI), 7(1), 114-138.

Sinnott, J. D. (1989). A model for solution of ill-structured problems: Implications for everyday and abstract problem solving. In J. D. Sinnott (Ed.), Everyday problem solving: Theory and applications (pp. 72-99). Praeger Publishers.

Spiesberger, P., Jungwirth, F., Wöss, C., Bachl, S., Harms, J., \& Grechenig, T. (2015, November). Woody: a location-based smartphone game to increase children's outdoor activities in urban environments. In Proceedings of the 14th International Conference on Mobile and Ubiquitous Multimedia (pp. 368-372). ACM.

Spradlin, D. (2012). Are you solving the right problem?. Harvard Business Review, 90(9), 84-+.

Syrjänen, A.-L. \& Kuutti, K. (2011). From technology to domain: The context of work for end-user development. In Proceedings of the 2011 iConference (pp. 244--251).

Tapia, E. M., Intille, S. S., \& Larson, K. (2004). Activity recognition in the home using simple and ubiquitous sensors. In International Conference on Pervasive Computing (pp. 158--175).

Truong, K. N., Huang, E. M., \& Abowd, G. D. (2004). CAMP: A magnetic poetry interface for end-user programming of capture applications for the home. In International Conference on Ubiquitous Computing (pp. 143--160).

Velicer, W. F., Prochaska, J. O., Bellis, J. M., DiClemente, C. C., Rossi, J. S., Fava, J. L., \& Steiger, J. H. (1993). An expert system intervention for smoking cessation. Addictive behaviors, 18(3), 269-290.

Von Hippel, E. \& Katz, R. (2002). Shifting innovation to users via toolkits. Management science, 48(7), 821--833.

Voss, J. F., \& Post, T. A. (1988). On the solving of ill-structured problems. In M. T. H. Chi, R. Glaser, \& M. J. Farr (Eds.) The nature of expertise. Hillsdale, NJ: Lawrence Erlbaum.

Vurgun, S., Philipose, M., \& Pavel, M. (2007). A statistical reasoning system for medication prompting. In International Conference on Ubiquitous Computing (pp. 1-18). 
Wakkary, R. \& Maestri, L. (2007). The resourcefulness of everyday design. In Proceedings of the 6th ACM SIGCHI conference on Creativity \& Cognition (pp. 163-172).

Wakkary, R. (2009). Anything Is a Fridge : The Implications of Everyday Designers. Interactions, 12-17.

Williams, R. S., Willard, H. F., \& Snyderman, R. (2003). Personalized health planning. Science, 300(5619), 549-549.

Wilson, S. (2003). The effect of music on perceived atmosphere and purchase intentions in a restaurant. Psychology of music, 31(1), 93-112.

Wood, W., \& Neal, D. T. (2007). A new look at habits and the habit-goal interface. Psychological review, 114(4), 843.

Xie, L., Antle, A. N., \& Motamedi, N. (2008). Are tangibles more fun?: comparing children's enjoyment and engagement using physical, graphical and tangible user interfaces. In Proceedings of the 2nd international conference on Tangible and embedded interaction (pp. 191-198). ACM.

Yilmaz, S. \& Seifert, C. M. (2011). Creativity through design heuristics: A case study of expert product design. Design Studies, 32(4), 384--415. 